University of Redlands

\title{
Web-GIS Services for Sustainable Ecotourism: Protected Areas in Northern Honduras
}

A Major Individual Project submitted in partial satisfaction of the requirements for the degree of Master of Science in Geographic Information Systems

by

Chisa Nishii

Mark P. Kumler, Ph.D., Chair

James A. Ciarrocca, M.S.

August 2007 
Web-GIS Services for Sustainable Ecotourism:

Protected Areas in Northern Honduras

Copyright (C) 2007

by

Chisa Nishii 
The report of Chisa Nishii is approved.
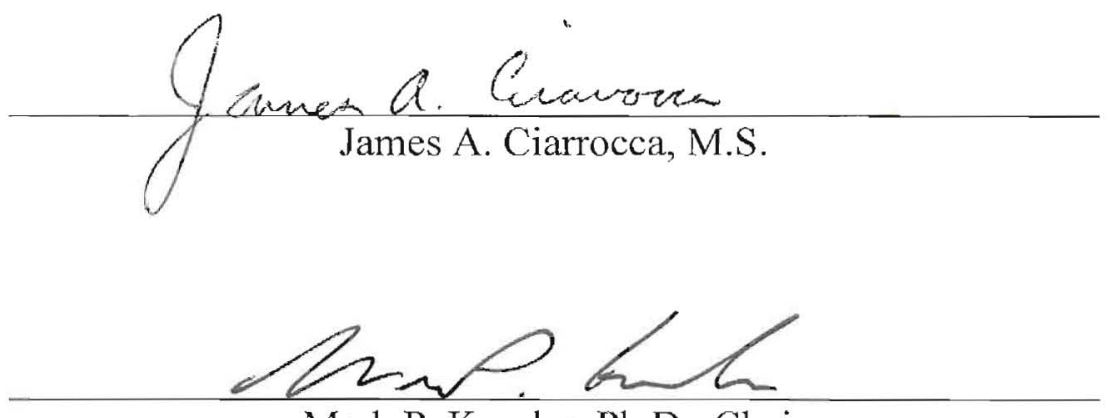

Mark P. Kumler, Ph.D., Chair

August 2007 


\section{ACKNOWLEDGEMENTS}

I would like to thank my parents in Guatemala, Kenichi and Fumiko Nishii, for trusting my interest in the subject and supporting my academic pursuits, and all the reasons for my being here. I would also like to thank Paul Duax and Mutsumi Nishii for accepting me for who I am, and for their support and understanding of my continuously changing pursuits in life.

I would like to thank Dr. Mark Kumler, committee chair, advisor and mediator, for his guidance and helpful advice. I am very appreciative for his supporting my pursuit for my Major Individual Project (MIP) from the beginning to end, and allowing me to shape this project the way I desired despite the many obstacles on the way.

I would like to thank Jim Ciarrocca, committee member and mentor, for his wisdom, encouragements and selfless comments that tremendously helped me in overcoming doubts and insecurity I confronted while working on this project. I am very grateful for his truly humble guidance and moral support in inspiring me to give my best effort.

This project would not have been possible without Theresa Ellis, the program coordinator who protected me from the "evil" distractions, which allowed me to focus on my MIP during crucial times. I would like to thank John Laska and Ruben Ortiz, the two wonderful doctors from the computer clinic who ensured my laptop was always healthy. I would also like to thank Lynn Flewelling and Ronald Pierre for their patience in editing my paper.

Thank you Cohort-10 for the many treasured hours we spent in the classroom-104 and the "gulag". I would like to thank Mike Cossey, the domain expert for programming I consulted very frequently. Thank you for your patience and friendship. I would like to thank Gina Durizzi, my food-marathon partner and a friend, who I admired from day one in the MS GIS Program, for illuminating my difficult moments with her million dollar smile and for being a significant part of my life in Redlands. I would like to thank Mathew Reidmiller, Tom Baker and Tim Leach for the many ideas, thoughts, and laughter we have shared. I will miss you all.

I feel sincerely grateful and fortunate to have shared this Masters Program with all these unique, wonderful and supportive individuals. 



\begin{abstract}
Web-GIS Services for Sustainable Ecotourism: Protected Areas in Northern Honduras

by

Chisa Nishii

Ecotourism is a promising source of economic growth in Honduras. The purpose of this project was to build a prototype website to disseminate tourist information online and to create a central geodatabase to facilitate and encourage the data entering, data processing, and data maintenance in the future. The project area encompasses two protected areas: Pico Bonito National Park and Cuero y Salado Wildlife Refuge. This prototype project implemented the ArcGIS 9.2 desktop application and ArcGIS Server 9.2 to (a) develop a web-GIS application to inform potential international and domestic tourists about the two protected areas, and (b) enable local participation in collecting data, creating, and maintaining a geodatabase to ensure consistent data entry and quality control for future ecotourism management. This paper describes data collection, geodatabase design, and the procedures undertaken to customize a website using Microsoft Visual Basic 2005 .NET development environment.
\end{abstract}

\title{
Key Words
}

Ecotourism, Protected Areas, northern Honduras, ArcGIS Server, web-GIS Application, Pico Bonito National Park, Cuero y Salado Wildlife Refuge 



\section{Table of Contents}

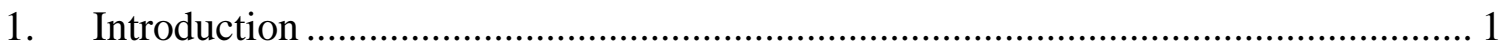

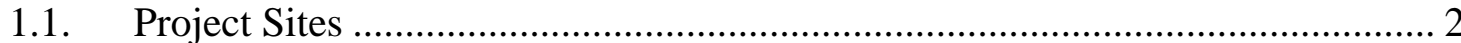

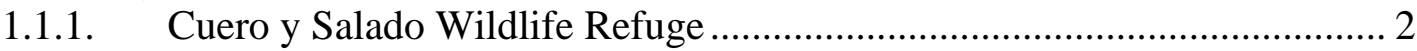

1.1.2. Pico Bonito National Park ...................................................................... 3

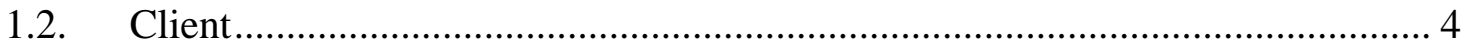

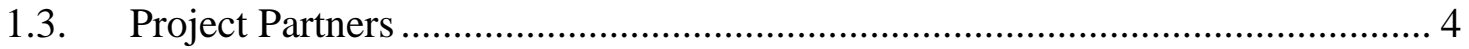

1.4. Problem Statement and Proposed Solution....................................................... 5

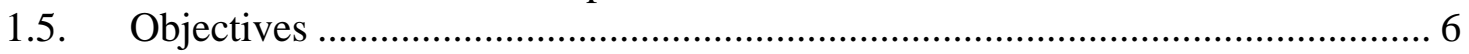

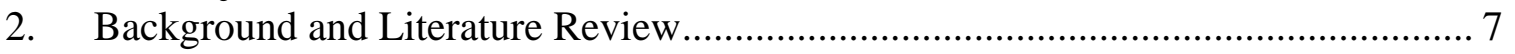

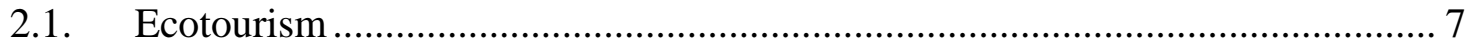

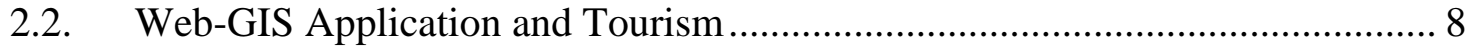

2.3. Example Web-GIS Applications............................................................. 9

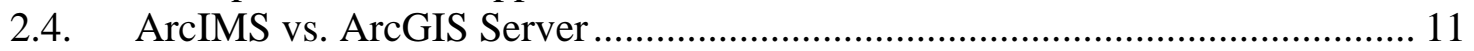

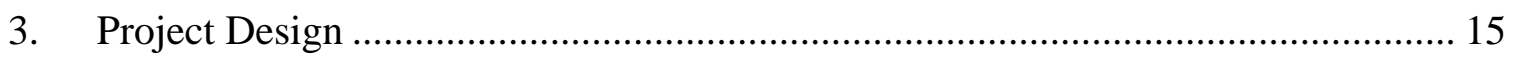

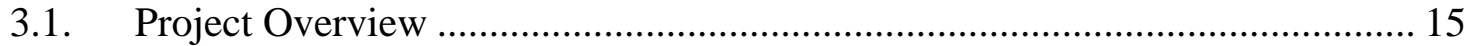

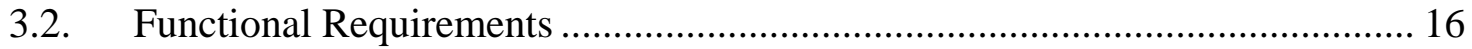

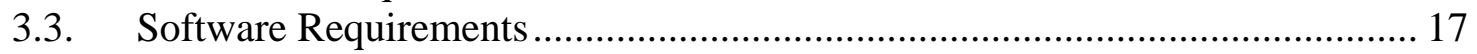

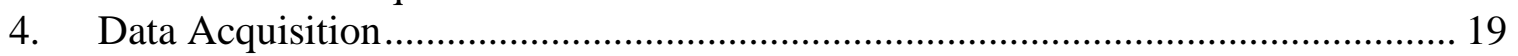

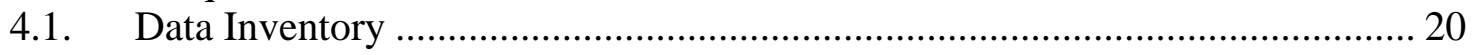

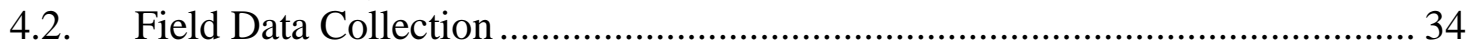

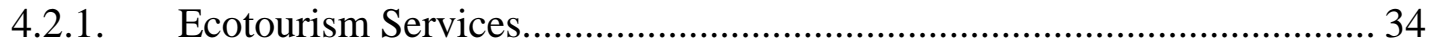

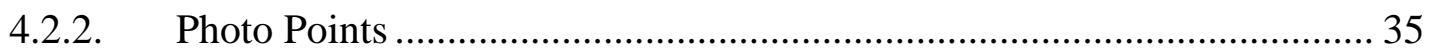

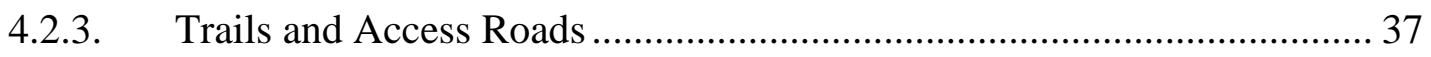

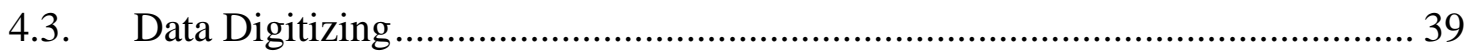

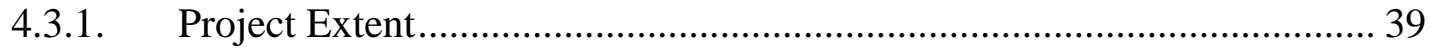

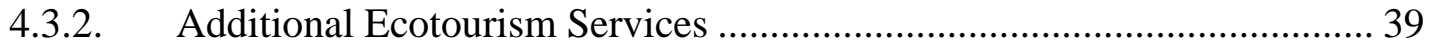

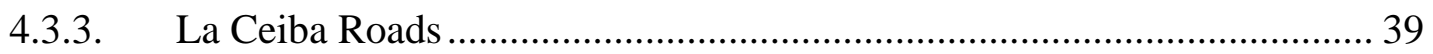

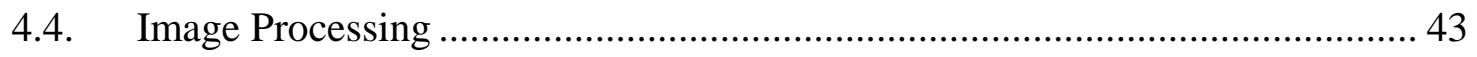

4.4.1. Creating the Swiss Hillshade .................................................................. 43

4.4.2. Creating the Multidirectional Oblique Weighting Hillshade.................... 44

4.4.3. Combining the Two Hillshades ................................................................. 44

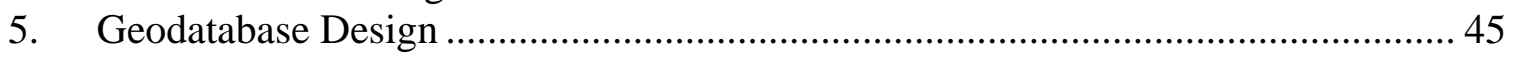

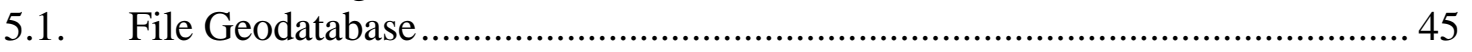

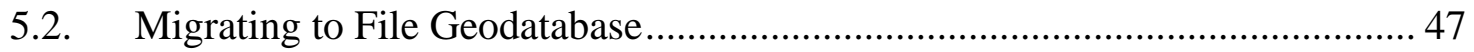

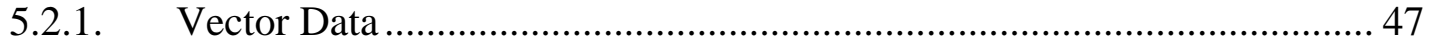

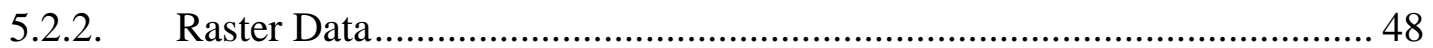

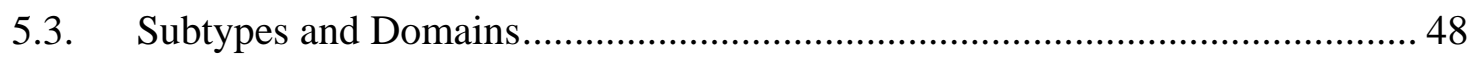

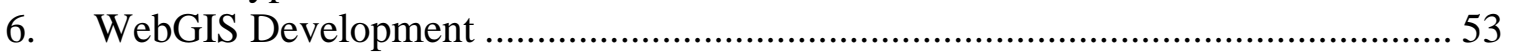

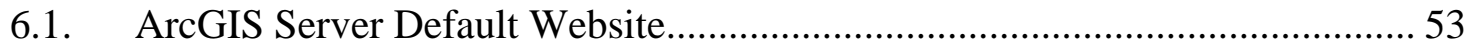

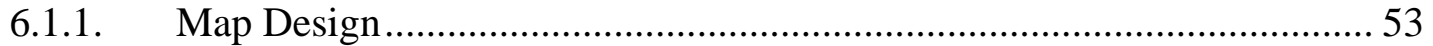

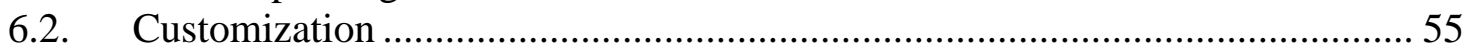

6.2.1. Custom Mapping Application Template.............................................. 56

6.2.2. Custom Table of Contents ................................................................. 57

6.2.3. Select and Zoom-In Functionality ...................................................... 58 


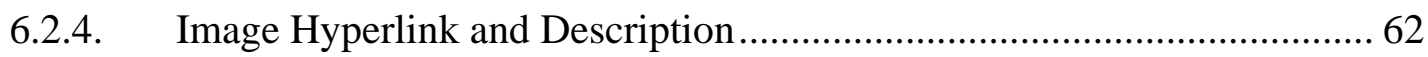

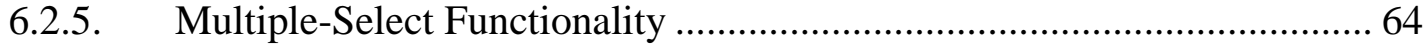

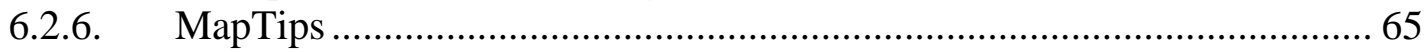

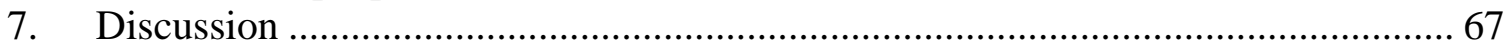

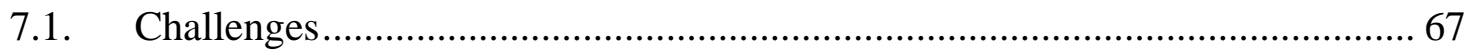

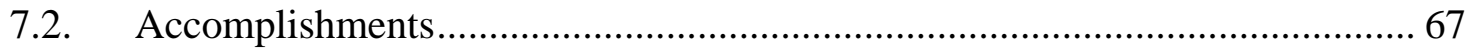

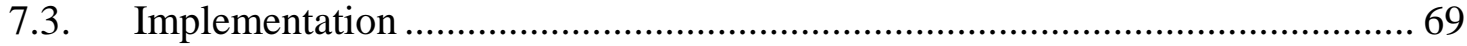

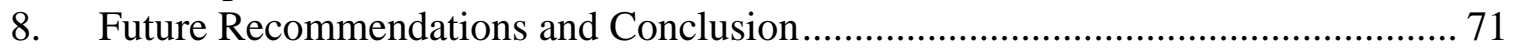

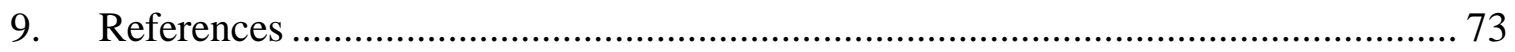

Appendix A - VBA Code: Microsoft Office Excel Data Catalog Utility Tool................ 75

Appendix B - Configuring the web.sitemap to Add External Links ............................... 81

Appendix C - Power Point Slide Show: Web-User Manual .......................................... 83

Appendix D - Power Point Slide Show: Project Overview............................................ 85

Appendix E - AJAX Enabled Custom Website Template Code: web.config ................... 87

Appendix F - Custom Table of Contents Code ................................................................ 93

Appendix G - Code for the Category Drop-Down List.................................................. 95

Appendix H - Code for the Subcategory Drop-Down List ......................................... 97

Appendix I - Another Code for the Subcategory Drop-Down List................................ 99

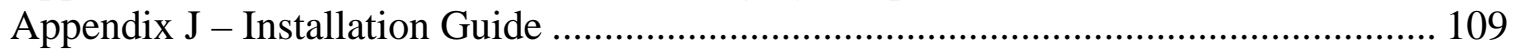




\section{Table of Figures}

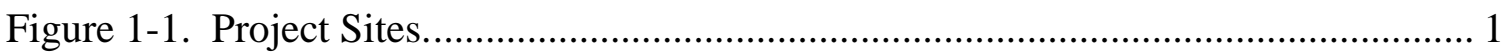

Figure 1-2. Cuero y Salado Wildlife Refuge. ……………........................................... 2

Figure 1-3. Pico Bonito National Park....................................................................... 3

Figure 1-4. Project Partners. ................................................................................. 5

Figure 2-1. Homepage for Tropical Andes Program. ………………….......................... 9

Figure 2-2. Homepage for the Santa Monica Green Map................................................. 10

Figure 2-3. Webpages from the ecoSports Website......................................................... 11

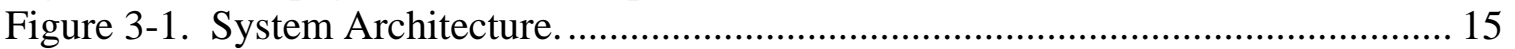

Figure 3-2. Software Requirements....................................................................... 17

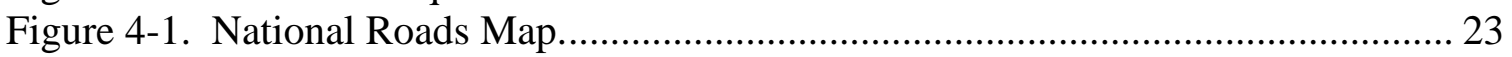

Figure 4-2. Regional Roads Map............................................................................. 24

Figure 4-3. National Rivers Map........................................................................... 25

Figure 4-4. Regional Rivers Map.......................................................................... 26

Figure 4-5. Forestry Map................................................................................... 27

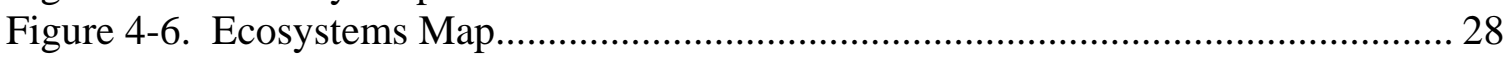

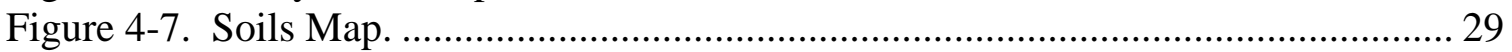

Figure 4-8. Life Zones Map..................................................................................... 30

Figure 4-9. Scanned Topo Map. ................................................................................ 31

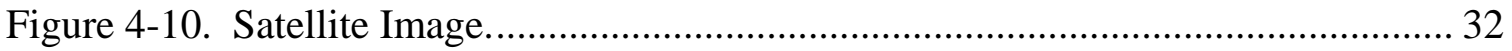

Figure 4-11. Digital Elevation Model......................................................................... 33

Figure 4-12. Ecotourism Services and Photo Points Map. …………………………….... 36

Figure 4-13. Trails and Access Roads Map................................................................... 38

Figure 4-14. Deriving Attribute Information from Travel Guide Books.......................... 39

Figure 4-15. La Ceiba City Tour Map. ........................................................................ 40

Figure 4-16. La Ceiba Lot Lines................................................................................ 41

Figure 4-17. La Ceiba Orthophoto Imagery. ............................................................. 41

Figure 4-18. La Ceiba Map................................................................................ 42

Figure 4-19. Swiss Hillshade Model......................................................................... 43

Figure 4-20. MDOW Hillshade Model Results............................................................. 44

Figure 4-21. Final Hillshade. ......................................................................................... 44

Figure 5-1. Characteristics of Geodatabase Types. ......................................................... 45

Figure 5-2. File Geodatabase Structure. ………………………………………….... 46

Figure 5-3. Ecotourism Services Feature Class and Subtype. ........................................... 49

Figure 5-4. Domains for Accommodation Subtypes. ........................................................ 50

Figure 5-5. Example of Subtypes and Domains in ArcMap Editor.................................. 51

Figure 6-1. Creating a Map Document for a Non-Custom Website.................................. 53

Figure 6-2. Visual Studio 2005 .NET Development Environment.................................... 56

Figure 6-3. List of Web Controls Used for the Customization......................................... 57

Figure 6-4. Default Table of Contents vs. Custom Table of Contents. ............................ 58

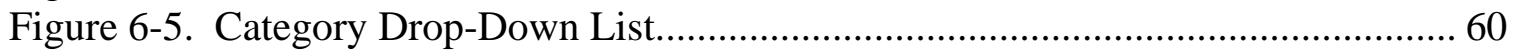

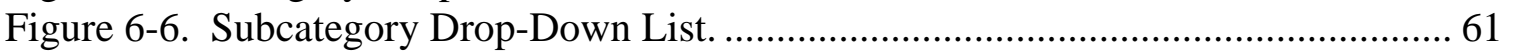

Figure 6-7. Zoom to Feature Functionality.................................................................... 62

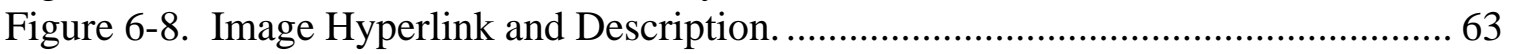

Figure 6-9. Multiple Select Functionality....................................................................... 65 


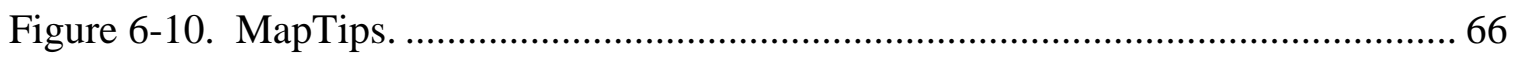

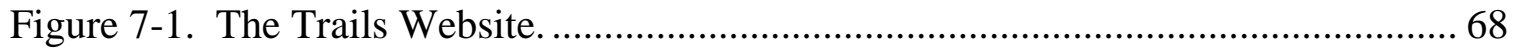

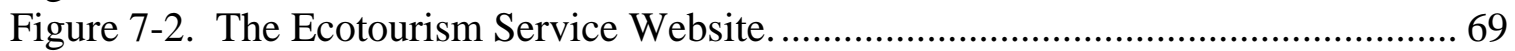

\section{List of Tables}

Table 2-1. List of Services Offered by ArcGIS Server................................................ 13

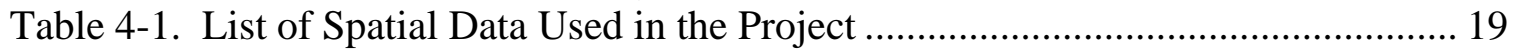

Table 4-2. Microsoft Office Excel Data Catalog Utility Tool with Customization......... 20

Table 4-3. Summary of Existing Data Inventory ......................................................... 21

Table 4-4. List of Ecotourism Service Location Types ............................................... 34

Table 5-1. List of Vector and Raster Data Migrated to File Geodatabase...................... 47

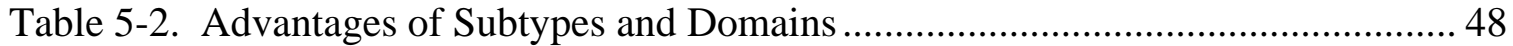




\section{List of Acronyms}

$\begin{array}{ll}\text { ADF } & \text { Application Developer Framework } \\ \text { CURLA } & \text { Centro Universitario Regional del Litoral Atlántico } \\ \text { DEM } & \text { Digital Elevation Model } \\ \text { ESRI } & \text { Environmental Systems Research Institute } \\ \text { FUCSA } & \text { Fundación Cuero y Salado } \\ \text { FUPNAPIB } & \text { Fundación para la Protección del Parque Nacional Pico Bonito } \\ \text { GIS } & \text { Geographic Information System } \\ \text { GPS } & \text { Global Positioning System } \\ \text { IDB } & \text { Inter-American Development Bank } \\ \text { IHT } & \text { Instituto Hondureño de Turismo } \\ \text { NGO } & \text { Non-Governmental Organization } \\ \text { MFH } & \text { Median Filter Hillshade } \\ \text { MIRA } & \text { Manejo Integrado de Recursos Ambientales } \\ \text { MDOW } & \text { Multidirectional Oblique Weighting } \\ \text { MS GIS } & \text { Master of Science in Geographic Information Systems } \\ \text { RCH } & \text { Raster Calculator Hillshade } \\ \text { REHDES } & \text { Red Ecologista Hondureña para el Desarrollo Sostenible } \\ \text { SINIA } & \text { Sistema Nacional de Información Ambiental } \\ \text { SVG } & \text { Scalable Vector Graphics } \\ \text { TNC } & \text { The Nature Conservancy } \\ \text { UNAH } & \text { Universidad Nacional Autónoma de Honduras } \\ \text { UNPD } & \text { United Nations Environment Programme } \\ \text { USAID } & \text { United States Agency for International Development } \\ \text { USGS } & \text { United States Geological Survey } \\ \text { UTM } & \text { Universal Transverse Mercator } \\ \text { VBA } & \text { Visual Basic for Application } \\ \text { WGS } & \text { World Geodetic System } \\ \text { WICE } & \text { World Institute for Conservation and Environment } \\ \text { WMS } & \text { Web Map Server } \\ \text { WWF } & \text { World Wildlife Fund } \\ & \end{array}$





\section{Introduction}

In 2004, the government of Honduras prioritized two protected areas in northern Honduras for potential ecotourism development: the core and buffer zones within the Pico Bonito National Park and Cuero y Salado Wildlife Refuge (Figure 1-1).

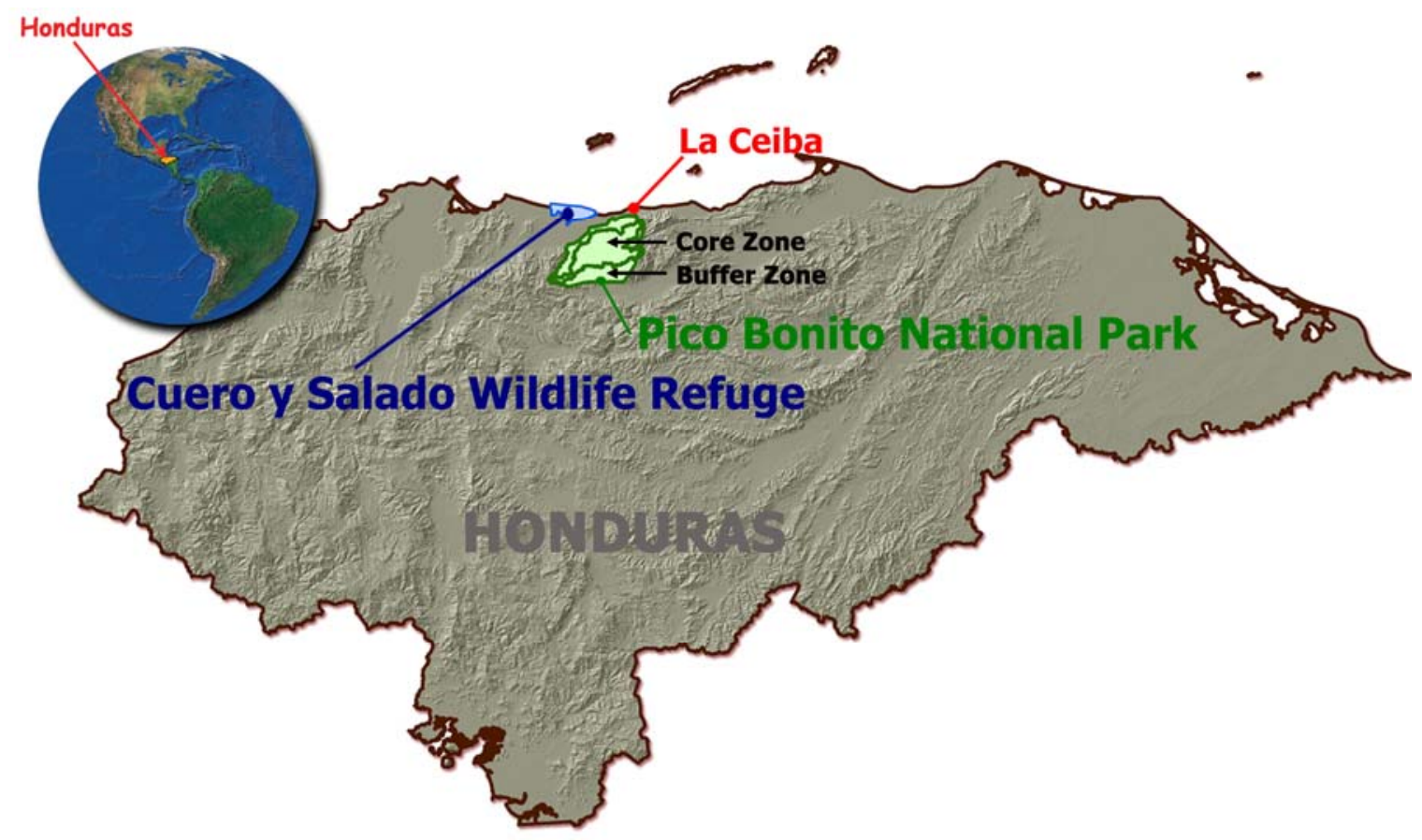

Figure 1-1. Project Sites.

The tourism sector of the protected areas is still in its early phase of development (Vreugdenhil, et al. 2002). In particular, lack of promotional materials available for the tourists is a problem. During a field visit to La Ceiba, Honduras (November 2006) and an informal interview with Leslie Alcántara, the Director of Ecotourism at the Pico Bonito National Park, the author was informed that visitors to the park keep asking for trails maps, "but we don't give them anything because... we don't have any!"

Viable ecotourism requires economic sustainability while maintaining environmental health. Creative advertising, such as posters, brochures, and Internet sites, is necessary to promote ecotourism in the region. Equally important is local community involvement in effective ecotourism management since "ecotourism is based on nature and will succeed only if nature remains in a relatively pristine state" (Boo, 1990). Currently, there is no central system that facilitates information sharing amongst decision makers responsible for ecotourism management in the protected areas, such as international and government agencies, municipalities, and environmental non-government organizations (NGOs).

Given the now widely utilized geographic information system (GIS) technology as a tool for natural resource management and dissemination of special data for information sharing, this prototype project implemented Environmental Systems Research Institute, Inc. (ESRI)'s GIS products. More specifically, the ArcGIS 9.2 desktop application and ArcGIS Server 9.2 were implemented to develop a web-GIS application to inform 
tourists about the two protected areas online, and to facilitate data entry and maintenance by creating a central geodatabase. This project is a prototype to support future management of the protected areas in Honduras.

\subsection{Project Sites}

Tourism in Honduras has particularly benefited from Caribbean cruise ships. Protected areas located on the north coast are very accessible from the cruise stops and are therefore important candidates for potential ecotourism destinations (Vreugdenhil, et al. 2002). The project site encompasses two protected areas, Cuero y Salado Wildlife Refuge and Pico Bonito National Park on the north coast, including the city of La Ceiba, the third largest city in Honduras. The protected areas of this project area were selected due in large part to their importance in the ecotourism industry in Honduras.

\subsubsection{Cuero y Salado Wildlife Refuge}

The Cuero y Salado Wildlife Refuge (Figure 1-2) is located 17 miles west of the city of La Ceiba, on the Caribbean coast.

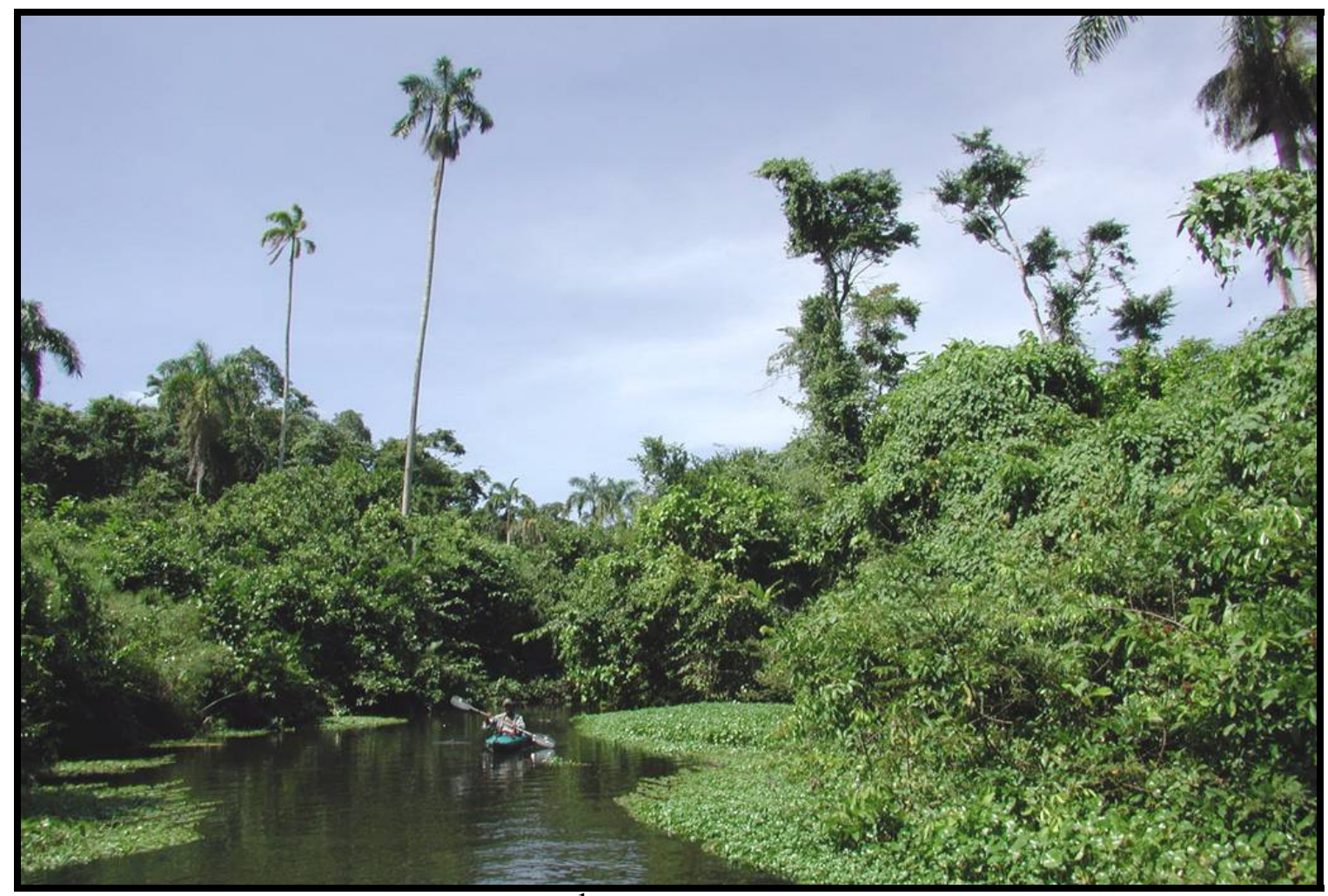

Figure 1-2. Cuero y Salado Wildlife Refuge ${ }^{1}$.

It is one of Honduras' smaller wetland reserves (13,225 hectares) managed by the Cuero and Salado Foundation (FUCSA) ${ }^{2}$. The refuge was established in 1987 primarily to protect manatees and their coastal wetlands and forest habitats, with technical and financial assistance from the local government and international agencies, including

\footnotetext{
${ }^{1}$ Image courtesy of FUCSA.

${ }^{2}$ Cuero y Salado Wildlife Refuge Homepage: www.cueroysalado.org
} 
United Nations Environment Programme (UNPD), Instituto Hondureño de Turismo (IHT), United States Agency for International Development (USAID), and other international conservation organizations (Wylie, 2001). The refuge has a recorded flora of more than 800 species (Vreugdenhil, et al. 2002). The refuge also has a great variety of migratory and residential birds, with some 196 species reported (Wylie, 2001). Approximately fifteen different rivers connect all parts of the Cuero y Salado Wildlife Refuge.

\subsubsection{Pico Bonito National Park}

Pico Bonito National Park is the second largest national park in Honduras (107,300 ha), and is located five miles south of the city of La Ceiba (Figure 1-3).

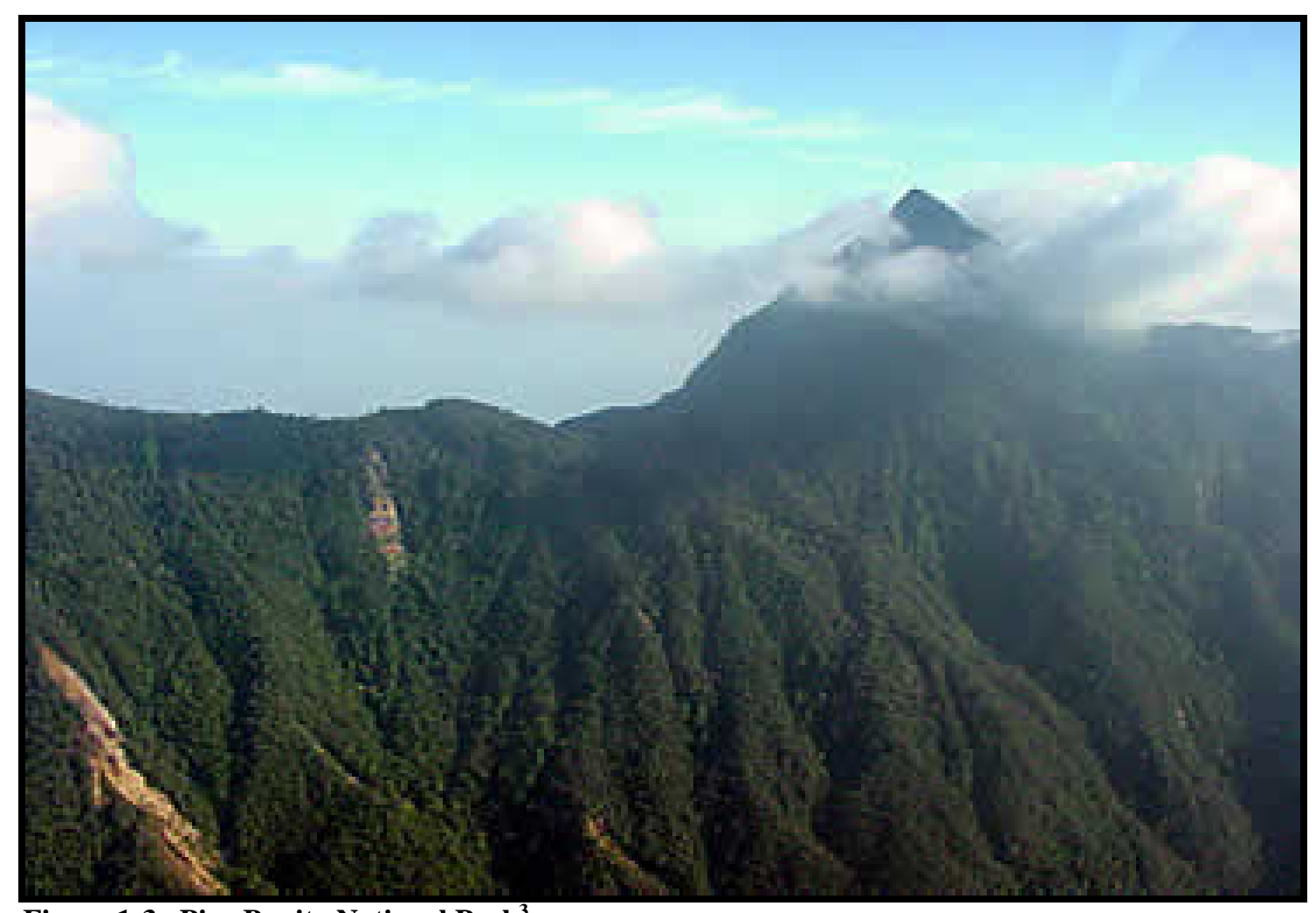

Figure 1-3. Pico Bonito National Park ${ }^{3}$.

This park consists of two zones: the core zone and the buffer zone. Although both zones are considered as protected areas, the core zone includes vulnerable ecosystems and is strictly protected, while the buffer zone "allows for certain form of utilization of natural resources” (Vreugdenhil, et al. 2002). The park is managed by the Pico Bonito National Park Foundation (FUPNAPIB) ${ }^{4}$, a private, non-profit organization, founded in 1993. It is one of the most diverse national parks with various ecosystem types ranging from tropical rainforest and broad leaf forest to cloud forests. The park is home to over 325 species of birds as well as jaguars, tapirs, deer, puma, white-faced and spider monkeys (Pico Bonito National Park, 2007).

\footnotetext{
${ }^{3}$ This image was retrieved from: http://www.honduras.com/parquepicobonito/index.htm

${ }^{4}$ Pico Bonito National Park Homepage: www.picobonito.org
} 
Approximately 6,000 tourists and researchers visited Pico Bonito National Park, and 3,000 visited the Cuero y Salado Wildlife Refuge in 2002 (Avila, 2004). The number of tourists visiting the protected areas is anticipated to increase.

\subsection{Client}

The client for this project is Dr. Robert Ford, Professor of International Sustainable Development and Social Policy, at Loma Linda University, in California. Dr. Ford collaborates with USAID - Honduran Office - to work on various projects in Honduras. In addition to collecting field data himself, Dr. Robert Ford trained local park staff and students from the local university, Universidad Nacional Autónoma de Honduras (UNAH), to collect pertinent field data for this project. He also acted as a liaison between the project partners in Honduras and the University of Redlands. As a liaison, he visited Honduras to determine their needs and, in turn, contacted the Master of Science in Geographic Information Systems (MS GIS) Program at the University of Redlands to inquire how GIS could satisfy those needs. Dr. Ford represented the project partners in deciding which data was most appropriate to use for this project.

\subsection{Project Partners}

The USAID is one of the international organizations collaborating with the government of Honduras, municipalities, environmental NGOs, and other stakeholders to support sustainable economic growth on the north coast of Honduras. Partial funding for this project was provided by USAID Honduras-USAID/MIRA (Manejo Integrado de Recursos Ambientales) project, which promotes ecotourism and sustainable development in Honduras.

Mr. Jose Herrero, the principal contact in Honduras for this project, is the director of the USAID/MIRA project. His primary interest is in generating employment opportunities within the Honduran community from the increased ecotourism in the region.

Dr. Robert Ford and Mr. Jose Herrero are the initiators of this project. In addition, there are several other current and prospective project partners who have contributed their enthusiasm toward the project and/or provided necessary data (Figure 1-4):

- United States Agency for International Development (USAID)

- $\quad$ Red Ecologista Hondureña para el Desarrollo Sostenible (REHDES) The Honduran Ecologist Network for Sustainable Development

- Fundación para la Protección del Parque Nacional Pico Bonito (FUPNAPIB) Pico Bonito National Park Management Unit

- Fundación Cuero y Salado (FUCSA) Cuero y Salado Wildlife Refuge Management Unit

- Instituto Hondureño de Turismo (IHT): Honduran Tourism Institute Department of Tourism in Honduras

- Sistema Nacional de Información Ambiental (SINIA) National System of Environmental Information

- Universidad Nacional Autónoma de Honduras (UNAH) National University in Honduras 
- Centro Universitario Regional Del Litoral Atlántico (CURLA)

Regional Center of the UNAH

- The Nature Conservancy (TNC)

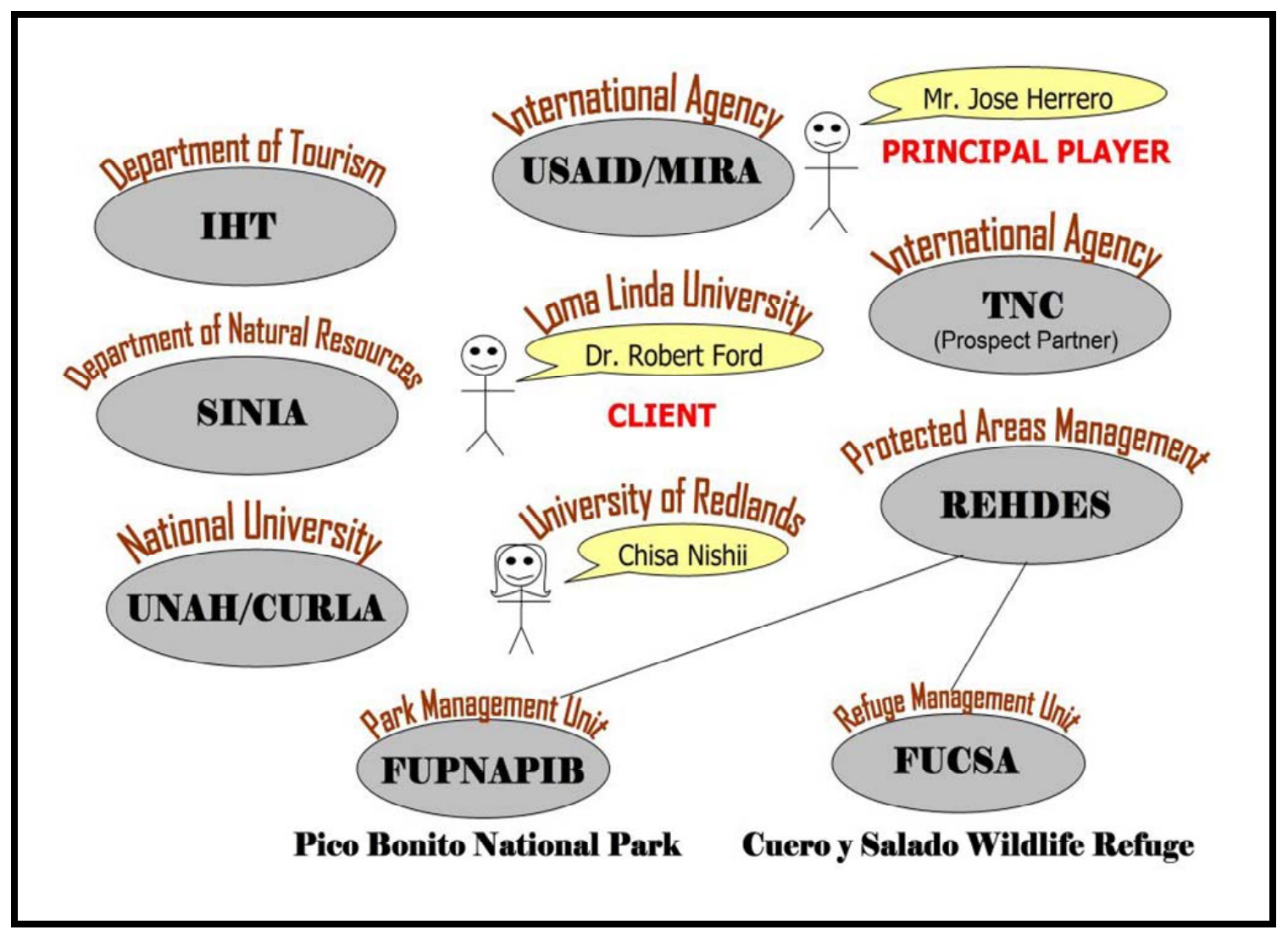

Figure 1-4. Project Partners.

\subsection{Problem Statement and Proposed Solution}

Lack of educational materials, guide books, maps, and promotional materials is a problem that the two protected areas are currently facing. The immediate problems regarding the creation of a GIS-enabled website were threefold: lack of funding, lack of data, and mismanagement of spatial data.

\section{Lack of Funding}

Case studies in ecotourism in the lesser developed world indicate that "protected area systems are chronically underfunded, despite their enormous indirect revenue-generating capacities” (Weaver, 1998), and the two protected areas of interest are no exception. Limited financial and personnel resources within the protected areas' administrative organizations is a problem. The two protected areas, Pico Bonito National Park and Cuero y Salado Wildlife Refuge, do not generate enough revenue to fund staff, provide for software training, or to supply equipment to develop web-GIS applications. Although each protected area has its own website, the park and refuge staff hope to upgrade their static maps to a more interactive map on the Internet. Building a prototype web-GIS application using ArcGIS server and exploring its capabilities solved this problem. 


\section{Lack of Spatial Data}

Tourists are interested in acquiring information regarding available hiking trails, tours, activities and events, locations of facilities, weather conditions, and bird watching sites in the protected areas. Unfortunately, there is a lack of spatial data specific to the protected areas to make trail maps or tourist maps that illustrate locations of restaurants, visitor centers, and lodging available for visitors. Data collection was the first step in creating a GIS-enabled website for tourists.

\section{Mismanagement of Data}

Some spatial data such as roads, rivers, vegetation, and land-use exists at the national level. However, little is known about the origin of the data and its accuracy. Data has been edited multiple times by many people over time. Multiple versions of the same thematic layer exist and no one knows which data is the most current. Inconsistent data collection is also a problem. Some attribute data are collected in Spanish and some are collected in English. Data compilation into a centralized database and database design was the proposed solution to support future data management.

\subsection{Objectives}

The focus of this project is the area surrounding the two protected areas: Pico Bonito National Park and Cuero y Salado Wildlife Refuge. The long-term goal of this project is to generate employment opportunities within the Honduran community while protecting the natural environment of the protected areas from the increased ecotourism in the region. The immediate goal of this project, however, is to develop a prototype website to distribute tourist information online and explore future tourist services. ESRI's GIS software was implemented to distribute spatial information online. Two GIS-enabled websites were developed to inform the potential tourists about the protected areas, including the natural environment of the area (e.g., soil composition, ecosystems, rivers), ecotourism services offered in the area, (e.g., hotels, ecolodges, restaurants, information centers, museums, banks) and locations of terrestrial and aquatic trails within the project site. GIS applications were implemented not only to display data, but also to support the decision-making process of the potential tourists as to where to stay overnight or dine when they visit the project site. This was accomplished by allowing Internet users to select hotels or restaurants under the user-specified price range varying from low to high. This project provided many opportunities to go well beyond the template offered by the ArcGIS Server 9.2. The particular interest towards the ArcGIS Server web application is based on technology improvement and effective advertisement media of Ecotourism in Pico Bonito National Park and Cuero y Salado Wildlife Refuge. 


\section{Background and Literature Review}

This chapter discusses key elements that comprise successful ecotourism and current international efforts in supporting sustainable ecotourism in Honduras, together with the problems that the protected areas in Honduras are currently confronting. In addition, this section addresses proposed solutions to the problem and why ArcGIS Server 9.2 was selected as a tool to develop the web-GIS application for this project. A literature search was conducted to explore current web-GIS applications on tourism industry, evaluate options, and select the solution. Furthermore, existing GIS-enabled websites were compared and examined in search of an optimal set of features and functionalities that could be useful for this project.

\subsection{Ecotourism}

Ecotourism is a promising source of economic growth in Honduras, primarily because it brings foreign currency into the local economies and, therefore, greatly increases national revenue. With a leading world-famous ecotourism campaign effort in Costa Rica, Honduras is also in the process of promoting its ecotourism to a broader audience, both domestic and international.

According to the International Ecotourism Society (2007), ecotourism is "responsible travel to natural areas that conserves the environment and sustains the well-being of local people." Successful ecotourism is achieved when there is a "mutually supporting partnership" among the following three elements (IUCN, 2006):

1. The natural environment

2. The local communities associated with the natural environment

3. The tourism system associated with the natural environment

Although small-scale in nature, this project attempts to comply with fundamental goals and requirements for a sustainable ecotourism. The overall objective of this project is to assess how GIS can be utilized to support ecotourism and establish a mutually rewarding relationship among participants responsible for the ecotourism management so that "the natural environment must benefit, communities must benefit, and the tourists and tourism businesses must benefit. Without this partnership, tourism may exploit local people and places and actually work against conservation” (IUCN, 2006).

Numerous international agencies have been developing strategies for successful ecotourism in Honduras. The World Wildlife Fund (WWF), for one, conducted a study in 1990 to investigate the nature-based tourism preference in the protected areas of Latin America. It was found that "when [tourists were] asked what could be improved, a majority mentioned educational materials, such as guide books, technical information, maps, promotional materials, and signs” (Boo, 1990). More recently, the World Institute for Conservation and Environment (WICE) conducted a thorough study in Honduras to analyze ecotourism opportunities in the protected areas. Immediate actions recommended by WICE for making the protected area more accessible for visitation included the "development of a marketing program for nature as a visitation destination" (Vreugdenhil, et al. 2002). As of 2007, there is a continuous effort by national and international agencies such as IHT, USAID, the World Bank, and Inter-American 
Development Bank (IDB) in supporting ecotourism development by building visitor centers and establishing various programs that promote environmental awareness and educational travel in the protected areas.

\subsection{Web-GIS Application and Tourism}

Web-GIS service is most recognized by its "ability to build robust applications and to share and integrate data and functionality over the World Wide Web” (Tang \& Selwood, 2003). According to Tang and Selwood, other web-GIS capabilities include (a) distribute and share the up-to-date spatial data, (b) facilitate the access, update and search of existing data, and (c) improve the maintenance and management of data. These capabilities are an integral part of this project. In particular, the information sharing component of the web-GIS services was crucial in disseminating tourist information online and sharing existing data among the local participants for more efficient planning and management of the natural resources and facilities within the protected areas.

Currently, there are GIS applications, not necessarily web-GIS applications, which bear directly on tourism management. According to the Knox's research project (2004), referencing W. Giles's GIS Applications in Tourism Planning (2003), GIS applications to tourism include:

- Recreational facility inventory

- Tourism-based land management

- Visitor impact assessment

- Recreation wildlife conflicts

- Mapping wilderness perceptions

- Tourism information management system and decision support systems

More specific examples include identifying sustainable trail development in a forest reserve in Sri Lanka (Boers, 2005), and analyzing suitable tourism hot-spots in the Currumbin Valley of Australia (Knox, 2004).

According to Balram \& Dragiâceviâc (2006), the web-GIS implementation in general is increasing and expanding across diverse areas including:

- Environmental decisions

- Data dissemination

- Environmental monitoring

- Public participation

- Community planning

Web-GIS using ESRI's Internet Map Server (ArcIMS) applications in data dissemination is particularly common and varies from disseminating regional information such as parks and recreational facility inventory in the Colorado Parks \& Recreation Department (Jensen \& Stonich, 2006), and wildlife and plant habitat for the Colorado Natural Diversity Information Source project (Bearly, et al. 1998), to a more global information, such as global warming data (Case \& Baker, 2003). Although not applied directly for the tourism industry, these project examples demonstrated the merit of utilizing web-GIS to the protected areas in Honduras. Implementing web-GIS not only facilitates the access and visualization of the existing data, but also facilitates the wide sharing of the data and 
effective use of the data, and therefore creates a more efficient system for the planning, management, and maintenance of the natural resources.

\subsection{Example Web-GIS Applications}

Many government agencies and private organizations have been utilizing the innovative web-based GIS technology to distribute spatial data online. Effective map displays and GIS functionalities are made available to the public. Below are three exceptional examples of web-GIS technology on the World Wide Web supporting ecotourism, environmental awareness, and park management. Web functionalities and layout designs of each website were closely examined in order to specify functional requirements for the web-GIS application built in this project.

\section{Tropical Andes Program}

The Programa Andes Tropicales is a private foundation that acts in favor of protecting the ecosystems in the Andes region in South America. Promoting rural tourism is only one of their many objectives. Through their web-mapping application using Scalable Vector Graphics (SVG), tourists can access spatial data, such as locations of parks, trails, and camp sites throughout Venezuela, Argentina, and Bolivia. Basic GIS functionalities (e.g., pan, zoom, print, query, identify) demonstrated in this website are useful. Their intention of engaging tourists with hands-on interaction on the computer screen is very clear by their hosting a site where the audience can download Global Positioning System (GPS) trail data. The "zoom to" drop down list is very intuitive and provides users with a good idea of existing campsites in the area (Figure 2-1). Although the visibility of the point symbols could be improved, this site was an excellent representation of what the project participants hoped to see on the final web-mapping application for this project.

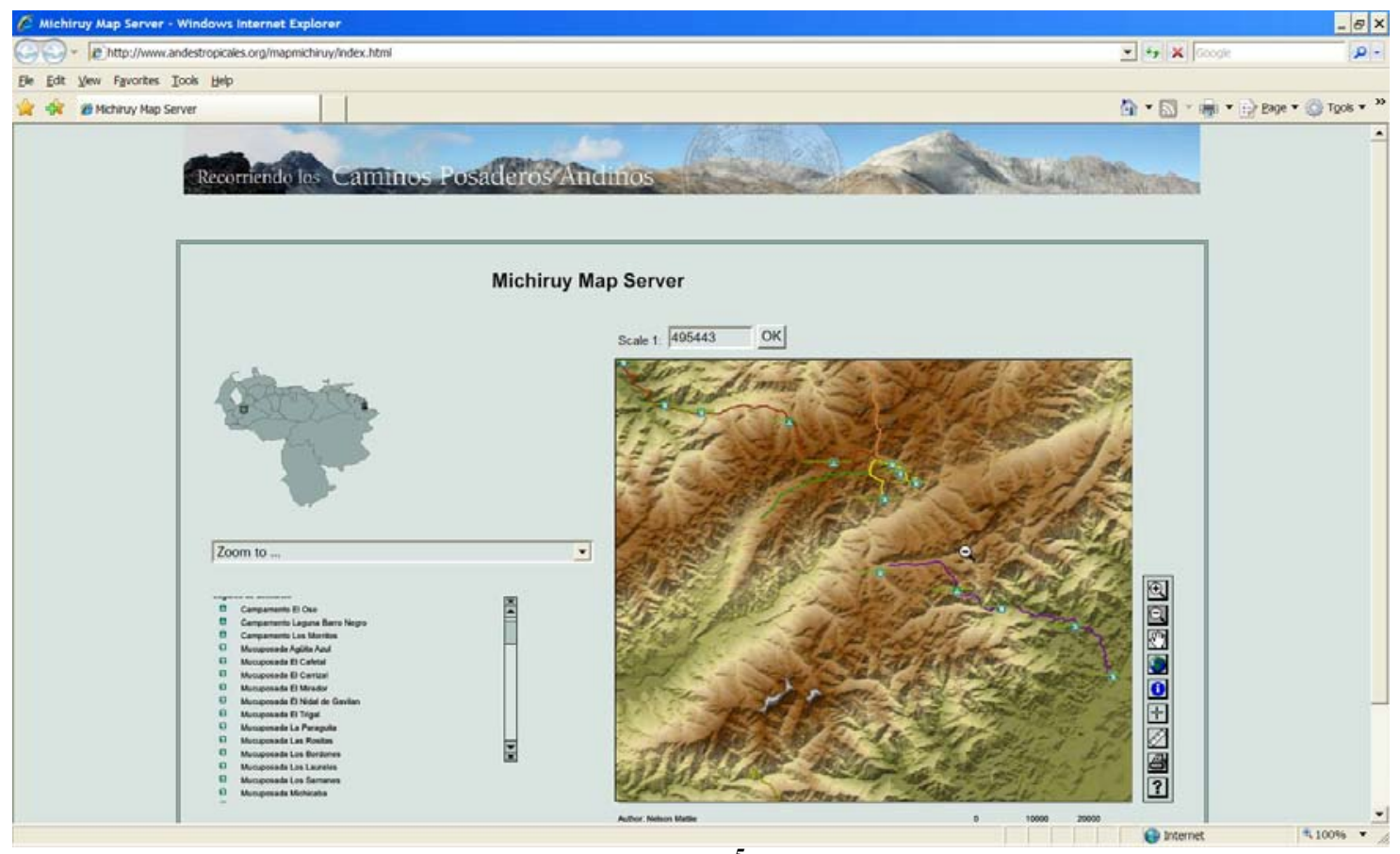

Figure 2-1. Homepage for Tropical Andes Program 5 .

\footnotetext{
${ }^{5}$ This map was retrieved from www.andestropicales.org by clicking on "Mapas”.
} 


\section{City of Santa Monica, California}

Santa Monica Green Map (Figure 2-2) was developed using GIS software and made public on the Internet to promote ecotourism in the area. More specifically, the Green Map on-line was created by the City of Santa Monica's Environmental Programs Division to promote environmental education for the community. Although not interactive, this online map allows the community to locate environmental organizations, vegetarian restaurants, natural food stores, and even locations of all storm water percolation systems, including statistics on the volume of water mitigated. This map was a collaborative effort of multiple stakeholders, including personnel from the Santa Monica High School Biology Department and Wildlife Club, the West Los Angeles chapter of the Audubon Society, the Santa Monica College Geo-Club, and many remarkable naturalists and business leaders. This online map conveyed that collaborative effort in creating an ecotourism map is achievable. The use of clear and colorful symbols and the visually pleasant layout design made this map very effective; these same design considerations were applied to the web-mapping application developed in this project.

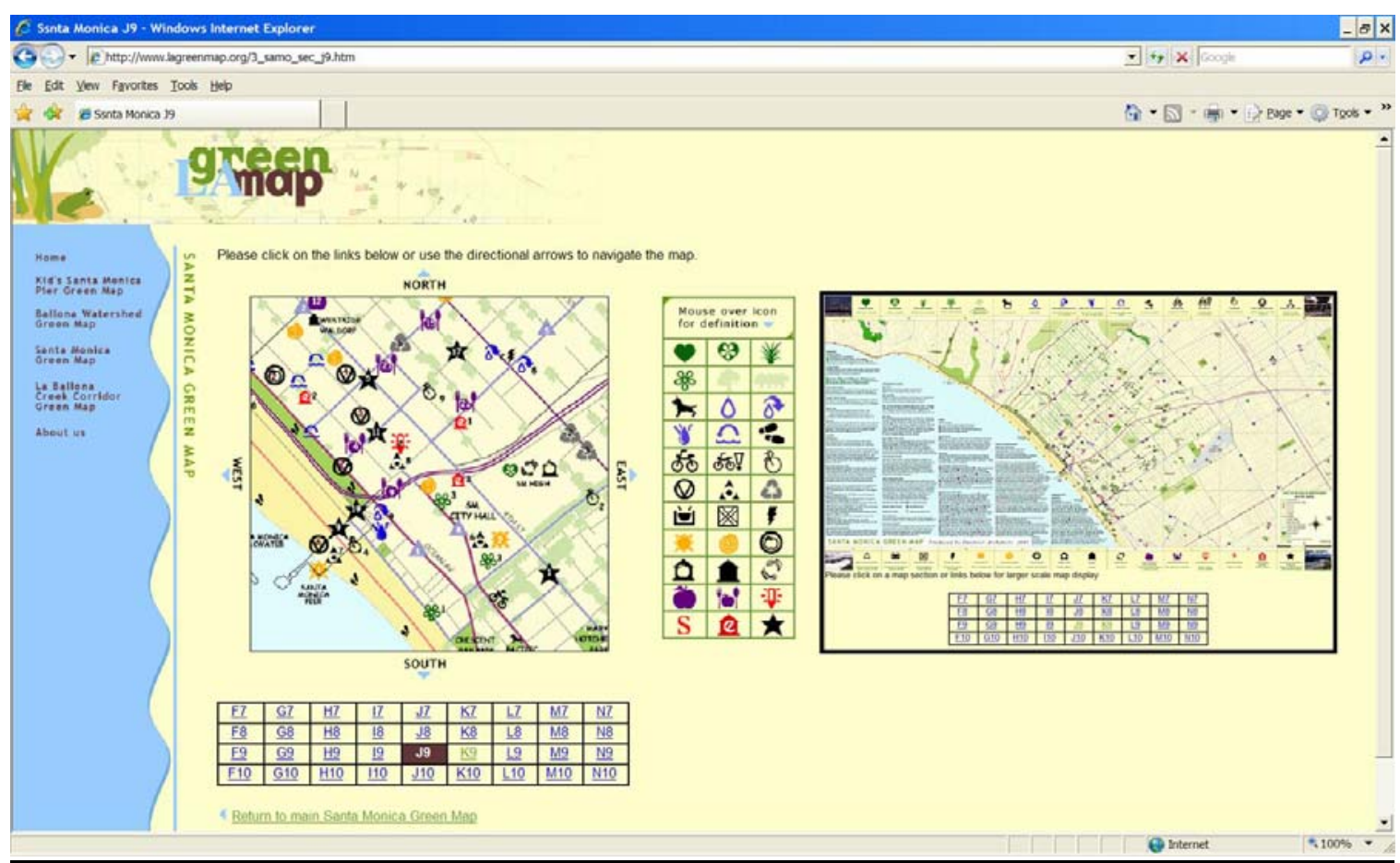

Figure 2-2. Homepage for the Santa Monica Green Map ${ }^{6}$.

\section{ecoSports:}

In their GIS-enabled website (Figure 2-3), ecoSports allows the audience to locate hiking trails, rafting waterways, and rock-climbing paths in Eagle County, Colorado according to their standardized level of difficulty: easy, moderate, and difficult. This was made possible by utilizing GIS analytical capabilities. This website is not only informative, but supports the decision making process of the target audience as to what hiking trail or

\footnotetext{
${ }^{6}$ This map was retrieved from www.lagreenmap.org/3_samo.htm by clicking on The Santa Monica Green Map.
} 
rock-climbing paths to consider. The cartography looks rather plain, but it facilitates finding the user-specific information. The cross-section display of each trail is unique and again helps the audience in determining which hiking trail to consider. Although the cross-section view of each trail was not incorporated in the website for this project, categorizing the trails by level of difficulty and allowing users to select trails of particular interest was implemented in this project.

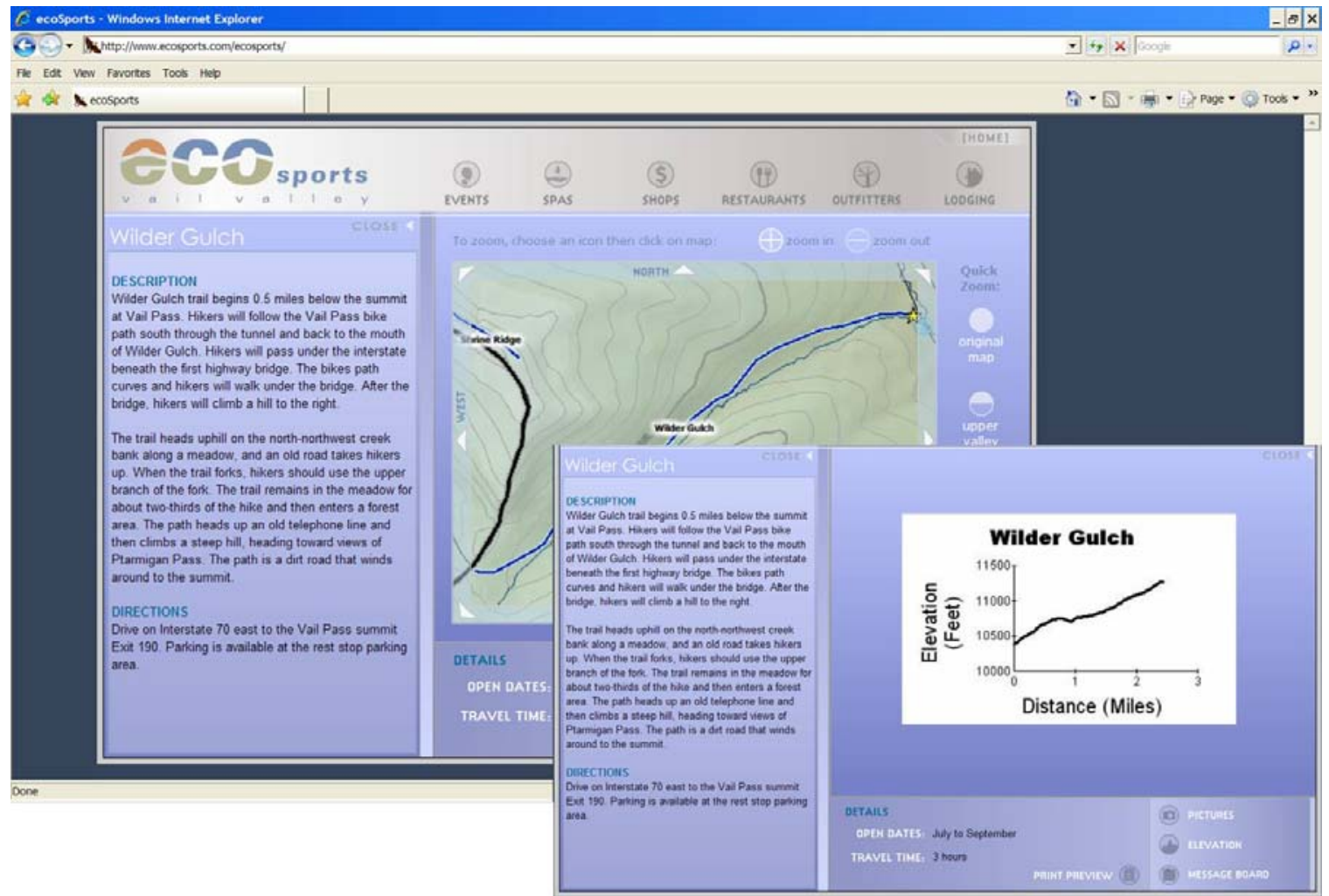

Figure 2-3. Webpages from the ecoSports Website ${ }^{7}$.

The integration of the web-based GIS for distributing tourist information online has been explored by various organizations to a certain extent, but not as widely as predicted. Finding websites that are spatially informative, interactive, quick, useful and fun, stable and reliable, and yet simple, intuitive, clear and readable is a challenge.

The three websites that were reviewed were good representations of what useful information represented over the Internet could be for an audience, using a web-GIS application. These example websites contributed various insights in designing a web-GIS application for this project. Specific functional requirements of the web-GIS application are discussed in more detail in Chapter 3.

\subsection{ArcIMS vs. ArcGIS Server}

One major decision was to choose between developing a web-GIS application using ESRI's traditional ArcIMS-based application, or the newest ArcGIS Server 9.2

\footnotetext{
${ }^{7}$ This map was retrieved from www.ecosports.com/ecosports by clicking on "HIKING."
} 
technology. The GIS-enabled websites presented in the previous sections utilized either the SVG or the ArcIMS technology. While ArcIMS solutions are used across a wide range of web-GIS applications and as data dissemination tools in various organizations across a wide variety of fields, ArcGIS Server has rarely been implemented. So far, it appears no attempt has been made to build a web-GIS application using ArcGIS Server 9.2 to promote ecotourism in any region of the world. This is primarily because webmapping application using ArcGIS Server is relatively new.

ArcGIS Server 9.2 is a development platform for building web-GIS applications that promotes the sharing of GIS data over the Internet. This application suits the objective goals of this project, not only by distributing tourist information online for potential visitors around the world, but also by sharing the most current information amongst decision makers who are responsible for the effective ecotourism management of the protected areas in Honduras. According to ESRI (2007a),

The main advantages of sharing GIS resources on a GIS server are the same as sharing any data through any kind of server technology: the data is centrally managed, supports multiple users, and provides clients with the most up-to-date information.

The benefit of using ArcGIS Server in comparison to ArcIMS is the added functionality. For instance, ArcIMS does not have capabilities of displaying spatial data in 3D. Editing data layers over the Internet is also a function available only in ArcGIS Server. Furthermore, the ArcGIS Server Web Mapping application supports display of multiple web mapping services such as ArcIMS sites, Web Map Server (WMS) services, and ArcWeb Services (ESRI, 2007b).

Web applications that can be built in ArcGIS Server vary from a very simple out-of-thebox website that uses pre-defined defaults settings, to a more advanced custom website with multiple functionalities including editing data online. Table 2-1 summarizes the three main services and the associated functionalities that ArcGIS Server offers: displaying data, providing functionality, and providing data. Although not all of the ArcGIS Server functionalities were utilized, the ArcGIS Server online mapping application was chosen over the ArcIMS application due to technology advancement and broader possibilities for future website development. 
Table 2-1. List of Services Offered by ArcGIS Server

DISPLAYING DATA
Map Services
-2D data visualization
Globe Services
- 3D data visualization
- 3D animation
Keyhole Markup Language (KML)
- Supports KML, the native format for Google Earth mapping service
Web Map Service (WMS)
- Supports external web map services
PROVIDING FUNCTIONALITY
Geoprocessing Services
- Exposes geoprocessing capabilities for web access
(buffer, clip, calculate travel cost surface, etc.)
Geocoding Services
- Enables Geocoding capabilities (type an address and receive a map)
Network Analysis Services
• Enables network-based spatial analysis
• Routing, travel directions, closest facility and service area analysis
PROVIDING DATA
Geodata Services
- Provides web access to data
- Executes database query
- Extracts data from the database
- Spobile Data Services

(ESRI, 2006a) 



\section{Project Design}

\subsection{Project Overview}

This section describes the system design, functional requirements, and software requirements that were incorporated in the project. The system architecture consists of three components (Figure 3-1): data acquisition, geodatabase design, and web GIS application. This project was designed as a three-phase process focusing on one component per phase. The entire system was built around ESRI's ArcGIS 9.2 desktop application and ArcGIS Server 9.2 technology.

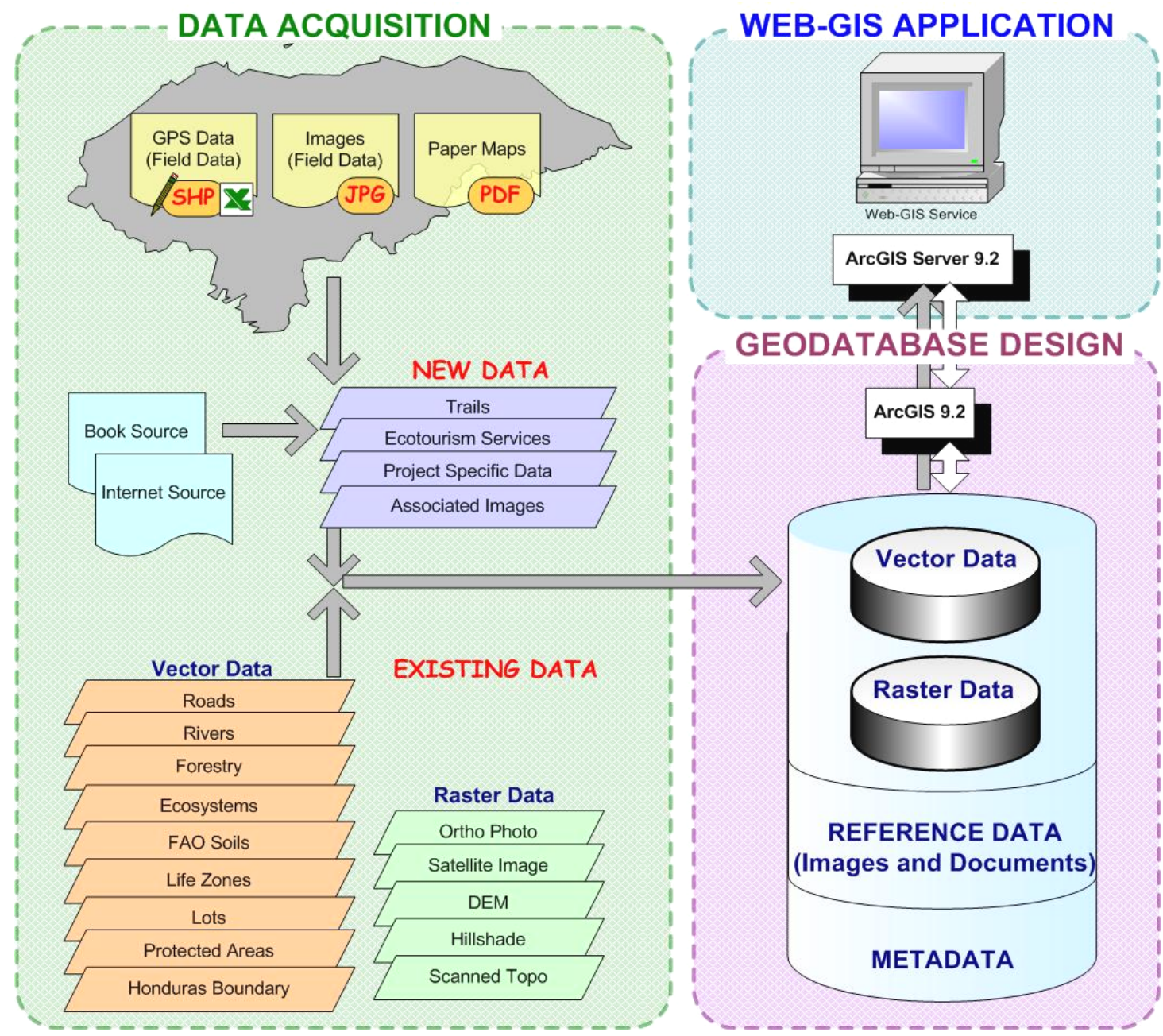

Figure 3-1. System Architecture.

\section{Phase One: Data Acquisition}

The logical first step was to identify spatial and non-spatial data pertinent to this project. First, data inventory on existing data was conducted. Field data then was collected in the protected areas. Field GPS data included location of hotels, restaurants, souvenir shops, information centers, museums, banks and many other facilities, together with trails and access roads. Finally, data was digitized from book and Internet sources for application 
development purposes. Chapter 4 discusses the data acquisition techniques and procedures in more detail.

\section{Phase Two: Geodatabase Design}

Building a website application that successfully displays data required the creation of a functional geodatabase. A file geodatabase was created to store spatial data in a logical and organized fashion so that users can easily add and retrieve data. Subtypes and domain were applied within the file geodatabase. The geodatabase integrated information from various sources, including existing data, newly collected field data, and digitized data. Chapter 5 discusses the geodatabase design in more detail.

\section{Phase Three: Web-GIS Application}

The next step focused on developing the web-GIS application. This web-GIS application was created to inform potential international and domestic tourists about the protected areas in northern Honduras by distributing spatial information online.

ArcGIS Server was utilized to build two GIS-enabled websites. First, a generic website was created using the default settings of the ArcGIS manager template. Then a custom website was created by customizing the ArcGIS manager template using the Visual Studio 2005 application by adding new functionalities such as drop-down lists, automatic zoom-in to selected features, and image display. The two websites inform the web visitors about protected areas, including the location of trails, restaurants, and souvenir shops around and within the protected areas. In addition, the website also informs the web visitors about the natural environment of the area. Chapter 6 discusses the details entailed in building and customizing a web-GIS application.

\subsection{Functional Requirements}

Below are functional requirements that were established to conduct this project.

\section{Geodatabase Design:}

- The geodatabase must intelligently synchronize spatial data from multiple sources:

o Field GPS data, tabular data and pictures collected in Honduras.

o Digitized data from books and Internet sources.

o Existing datasets.

- All vector and raster data in the geodatabase must have the same projection: UTM Zone 16N WGS1984.

- All vector and raster data in the geodatabase must have metadata.

- Tabular data must have consistent naming convention.

- The domain table must be editable in Excel Spreadsheet to accommodate clients' needs.

- Subtypes, domains, topology, and relationships must be clearly defined.

- Editable and non-editable data must be clearly distinguished in the geodatabase. 


\section{Web-GIS Services:}

The web interface will inform the audience about the protected areas by displaying the following:

- An interactive map of the project site.

- Table of contents that lists all the visible layers.

- Data layers specified by the client.

- Data layers with scale dependencies.

- Links to external websites.

- Drop down lists with pre-defined tourist services to choose from.

- Drop down lists with pre-defined trails to choose from.

The web interface will provide the Internet users with the following functionalities:

- Basic tools (e.g., pan, zoom-in, zoom-out, identify, measure).

- Turn on and off layers of interest.

- Click on the photo points and retrieve associated images and information.

- Auto zoom functionality into the user-specified ecotourism service location.

- Auto zoom functionality into the user-specified trail.

The web interface will allow Internet visitors to perform simple queries, such as:

- Select trails in a particular protected area.

- Select trails of particular difficulty level: easy, medium, or difficult.

- Select individual trail and view associated description and image.

- Select individual ecotourism services and view associated description and image. (e.g., hotels, restaurants, information centers, museums, banks, clubs)

- Select multiple hotels in a specific price range.

- Select multiple restaurants in a specific price range.

\subsection{Software Requirements}

This project incorporated both the ArcGIS 9.2 desktop application with ArcINFO license and ArcGIS Server 9.2 on one individual hardware system. Figure 3-2 demonstrates the various software installed for the use of this project.

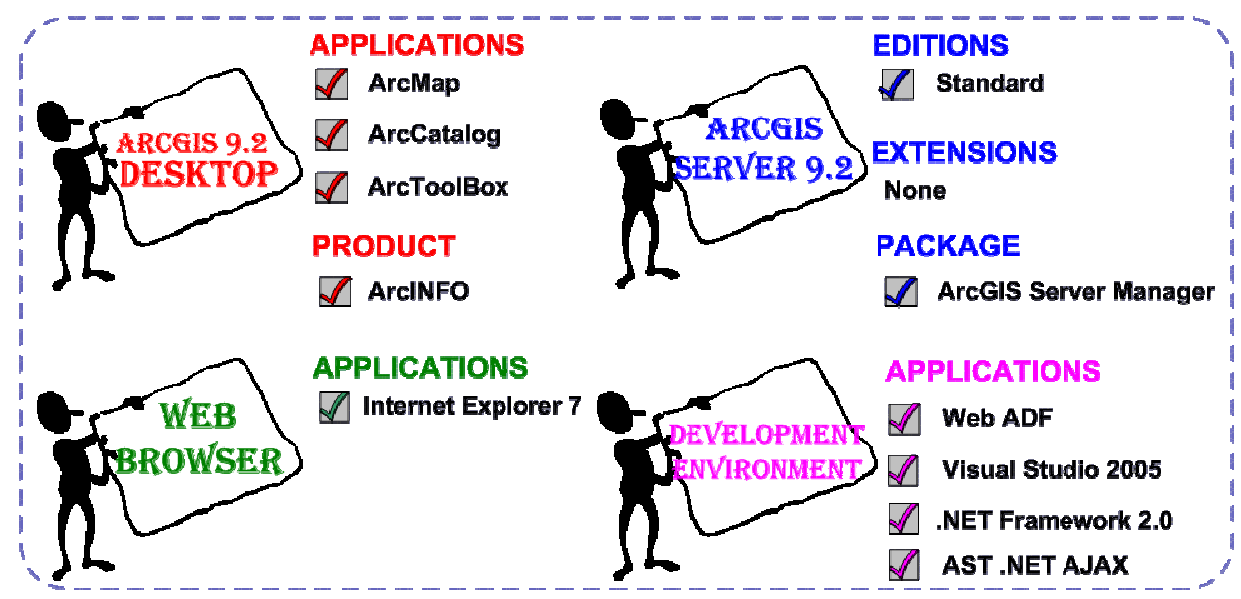

Figure 3-2. Software Requirements. 


\section{ArcGIS 9.2 Desktop Application}

ArcMap 9.2 was used to create the base map and to store data in the file geodatabase. ArcToolBox was used to create the geodatabase, mainly to project data sets and apply subtypes and domains into the geodatabase. The base map was published online in ArcCatalog. Metadata for each feature class was added in the ArcCatalog.

\section{ArcGIS Server 9.2}

ArcGIS Server version 9.2 (standard edition) was installed on an individual laptop equipped with PC-Intel Windows XP Professional Edition. No extension was used for this project. Further information on system requirements for ArcGIS Server is found on the ESRI's ArcGIS Server webpage ${ }^{8}$. ArcGIS Server Manager was used as an alternative method of publishing the map documents for this project.

\section{Web Browser}

Internet Explorer 7 was used throughout the development of the web-GIS application for this project. Internet users with Internet Explorer 6 will be able to view the final websites, but not with earlier versions. The complete list of supported web browsers for ArcGIS Server 9.2 is found on the ESRI's ArcGIS Server webpage ${ }^{8}$.

\section{Development Environment}

Custom web-mapping application was developed using the Microsoft Visual Studio 2005 .NET development environment. Web Server must have .NET 2.0 run-time environment in order to host a website. The Microsoft ASP .NET AJAX extension was downloaded and implemented to improve the website performance and display.

\footnotetext{
${ }^{8}$ ArcGIS Server webpage: http://www.esri.com/software/arcgis/arcgisserver/
} 


\section{Data Acquisition}

Essential to this project were spatial datasets that inform the potential visitors about the protected areas. This chapter describes in greater detail the process of data acquisition.

Data acquisition was conducted throughout several months, from December 2006 to April 2007, with the initial phase being the identification of available spatial data necessary for the project, followed by field data collection in conjunction with data digitizing. This chapter documents each of these three separate data acquisition components in detail.

Spatial datasets used for this project included existing data, digitized data, and newly collected GPS data. Table 4-1 lists all spatial datasets used for this project with corresponding feature type, data capture method(s), and figure number.

Table 4-1. List of Spatial Data Used in the Project

\begin{tabular}{|l|l|l|l|}
\hline \multicolumn{1}{|c|}{ Vector Data } & \multicolumn{1}{c|}{$\begin{array}{c}\text { Feature } \\
\text { Type }\end{array}$} & \multicolumn{1}{c|}{ Data Capture Method } & \multicolumn{1}{c|}{ Figure } \\
\hline Project Area Boundary & Polygon & Existing data inventory & All Figures Below \\
\hline Project Extent & Polygon & Digitizing & All Figures Below \\
\hline National Roads & Line & Existing data inventory & Figure 4-1 \\
\hline Regional Roads & Line & Existing data inventory & Figure 4-2 \\
\hline National Rivers & Line & Existing data inventory & Figure 4-3 \\
\hline Regional Rivers & Line & Existing data inventory & Figure 4-4 \\
\hline Forestry & Polygon & Existing data inventory & Figure 4-5 \\
\hline Ecosystem & Polygon & Existing data inventory & Figure 4-6 \\
\hline FAO Soils & Polygon & Existing data inventory & Figure 4-7 \\
\hline Life Zone & Polygon & Existing data inventory & Figure 4-8 \\
\hline Ecotourism Services & Point & Digitizing/GPS data collection & Figure 4-12 \\
\hline Photo Points & Point & GPS data collection & Figure 4-12 \\
\hline Access Roads & Line & GPS data collection & Figure 4-13 \\
\hline Trails & Line & Digitizing/GPS data collection & Figure 4-13 \\
\hline La Ceiba Boundary & Polygon & Digitizing & Figure 4-18 \\
\hline La Ceiba Lots & Polygon & Existing data inventory & Figure 4-18 \\
\hline La Ceiba Roads & Line & Digitizing & Figure 4-18 \\
\hline & Feature & \multicolumn{1}{|c|}{ Data Capture Method } & \\
\hline Raster Data & Type & Figure \\
\hline Scanned Topo Image & Raster & Existing data inventory & Figure 4-9 \\
\hline Digital Elevation Model (DEM) & Raster & Existing data inventory & Figure 4-10 \\
\hline Orthographic Photography & Raster & Existing data inventory & Figure 4-11 \\
\hline Hillshade & Raster & Existing data inventory & Figure 4-18 \\
\hline
\end{tabular}




\subsection{Data Inventory}

The first step in data acquisition was the identification of base layers, including the protected areas' boundary, roads, rivers, vegetation type, ecosystems, life zones, soil types, and background images. Initial efforts by the client resulted in the collection of more than 60,000 documents (approximately 90GB) from various organizations in Honduras. However, despite an extensive search, no records pertaining to the data collection activities were located. The lack of inventory of historical records required an extensive search and identification of all existing datasets and related documentation.

The Microsoft Office Excel Data Catalog Utility tool on the ESRI's ArcScript website ${ }^{9}$ was utilized to conduct an automated catalog of three most recent directories with documents collected by the client in Honduras.

The Visual Basic for Application (VBA) script embedded in the tool performs a search based on the user's specified directory and extracts detailed information on each object within the directory (Table 4-2), including the object location, name, type, and number of rows (see (1). The original VBA script was slightly modified (Appendix A) so that the tool could extract additional information about the objects, including projection and feature type, if applicable (see (2).

Table 4-2. Microsoft Office Excel Data Catalog Utility Tool with Customization

\begin{tabular}{|c|c|c|c|c|c|c|}
\hline 1 & $\begin{array}{c}\text { A } \\
\text { Container }\end{array}$ & $\begin{array}{c}\text { B } \\
\text { Object }\end{array}$ & $\begin{array}{c}\mathrm{C} \\
\text { Object Type }\end{array}$ & $\begin{array}{c}\text { D } \\
\text { \#Rows }\end{array}$ & $\begin{array}{c}E \\
\text { Projection }\end{array}$ & $\begin{array}{c}F \\
\text { Feature Type }\end{array}$ \\
\hline & C.MSGIS COHORT10MIPIDATAIHONDURASIDR FORDI03232007Pico Bonito & & & & & \\
\hline 3 & 1 & Hond_032107_proi_shp_shp & Shapefile & 250 & WGS_1984_UTM_ZOne_16N & Point \\
\hline 4 & 1 & Hond_032107_unp_shp_shp & Shapefile & 250 & GCS_WGS_1984 & Point \\
\hline 5 & 1 & Hond trac 032107 proi sho she & Shapefile & 250 & WGS 1984 UTM Zone $16 \mathrm{~N}$ & Point \\
\hline 6 & 1 & Hond trac_032107_proi_shp_line.shp & Shapeflie & & WOS_1984_UTM_Zone_16N & Polyline \\
\hline 7 & 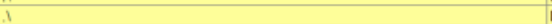 & Hond trac 032107 pro_ shp_point shp & Shapefile & $128 \mathrm{~A}$ & WGS_1984_UTM ZOne-16N & Point \\
\hline 8 & & Hond_trac_032107_unp_shpsshp & Shapeflie & $25 \mathrm{a}$ & GCS_WGS_1984 & Point \\
\hline 9 & & Hond trac_032107_unp_shp line shp & Shapeflie & & GCS_WGS_1984 & Polyline \\
\hline 10 & LarciMS Project Pico Bonito & bejuco.jpg & JFIF : 3 band(s) & NA & Unknown & \\
\hline 11 & AarcilMS Project_Pico_Bonito & h1605.jpg & JFIF : 3 band(s) & NA & Unknown & \\
\hline 12 & ArcIMS_Project_Pico_Bonito & $\mathrm{mn} . \mathrm{jpg}$ & JFIF : 3 band(s) & NA & Unknown & \\
\hline 13 & ArciMS_Projoct_Pico_Bonito & ubicación del proyecto.jpg & JFIF : 3 band(s) & NA & Unknown & \\
\hline 14 & ArciMS Project Pico Bonto & crtint & TIN Dataset & NA & & \\
\hline 15 & ArciMS Project Pico_Bonito & no boca cerrada shp & Shapefile & & Unknown & Polygon \\
\hline 16 . & ArciMS Project Pico_Bonito & xtdofaults bt & Text File & & Unknown & Polygon \\
\hline 17. & ArcIMS Projoct Pico Bonito Boca Corrada & boca cerrada puntos shp & Shapefile & $50 \pi$ & Unknown & Point \\
\hline 18. & ArcIMS Project Pico Bonito Boca Cerrada & camino boca cerrada shp & Shapefile & 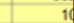 & Unknown & Polyline \\
\hline 19 & ArcIMS Project Pico Bonitol Boca Cerrada & el sitio camino shp & Shapefile & & Unknown & Polyline \\
\hline 20. & ArciMS_Project_Pico_BonitolBoca Cerrada & el sitio puntos ship & Shapefile & 19 & Unknown & Point \\
\hline 21 . & AarcIMS Project Pico Bonito Boca Cerrada & Boca Cerriada dof & dBASE Tabie & 507 & Unknown & Point \\
\hline 22 . & ArcIMS Project_Pico_BonitolBoca Cerrada & Boca Cerrada xls & Excel File & NA & & \\
\hline 23 . & ArciMS Project Pico Bonito Boca CerradaiBoca Cerrada xls & Sheet1s & Excel Table & $50 \%$ & & \\
\hline 24 . & ArcIMS Project Pico_Bonito Boca CerradaiBoca Cerrada.xls & Sheet2s & Excel Table & & & \\
\hline 25 . & ArcIMS Project_Pico_Bonito Boca Cerrada Boca Cerrada. xls & Sheet3s & Excell Table & & & \\
\hline 26 . & ArcIMS_Project Pico_Bonito Boca CerradaMopas & boca cerrada.jpg & JFIF : 3 band(s) & NA & Unknown & \\
\hline 27. & ArcIMS_Project_Pico_BonitolBoca CerradalMapas & boca cerrada2.jpg & JFIF : 3 band(s) & NA & Unknown & \\
\hline 28 & ArciMS Project Pico BonitoiCamp CURIA & curla jipg & JFIF $: 3$ band(s) & NA & Unknown & \\
\hline 29 & ArciMS_ProjectPico_BontolCamp CURLAiEl Bejuco CURLA & bejuco curla puntos shp & Shapeflie & 20 & Unknown & Point \\
\hline 30 & AarciMS_Project_Pico_BontolCamp CURLAiEI Bejuco CURLA & bejucocurla shp & Shapefile & & Unknown & Polyline \\
\hline 31. & AarciMS Project Pico BontolCamp CURLAIEI Bejuco CURLA & Bejuco CURLA1 dbt & dBASE Table & 20 & Unknown & Polyline \\
\hline 32 & AarcIMS Project Pico BontolCamp CURLAIEI Bejuco CURLA & Bejuco CURLA xis & Excel Fille & NA & & \\
\hline 33 & AarcIMS Project Pico BonitoiCamp CURLAIEI Bejuco CURLALBejuco CURLA uls & s sheet1s & Excel Table & 5. & & \\
\hline 34 & ArcIMS_Project_Pico_BonitolCamp CURLAIEI Bejuco CURLABBejuco CURLA vis & s Sheet2s & Excel Table & & & \\
\hline 35 & 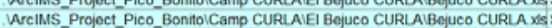 & sheet3s & Excel Table & & & \\
\hline 36 & ArcIMS Proect Pico BontoiCamp CURLALLa Guatuza & quatura shp & Shapefile & & Unknown & Polyline \\
\hline
\end{tabular}

Approximately 15,000 objects were cataloged in this process (Table 4-3). Among these, approximately 300 personal geodatabase feature classes and 1300 shapefiles were closely observed to specify spatial data to distribute online. Unfortunately, none of the personal geodatabase feature classes were within the spatial extent of the project area.

\footnotetext{
${ }^{9}$ ESRI's ArcScript website: http://arcscripts.esri.com/
} 
Table 4-3. Summary of Existing Data Inventory

\begin{tabular}{|c|c|}
\hline Count of Format & \\
\hline Format & Total \\
\hline Annotation Feature Class & 1700 \\
\hline Arc Feature Class & 1553 \\
\hline BMP : 1 band(s) & 36 \\
\hline BMP : 3 band(s) & 13 \\
\hline CAD Annotation Feature Class & 57 \\
\hline CAD Drawing & 57 \\
\hline CAD Feature Dataset & 57 \\
\hline CAD MultiPatch Feature Class & 57 \\
\hline CAD Point Feature Class & 57 \\
\hline CAD Polygon Feature Class & 57 \\
\hline CAD Polyline Feature Class & 57 \\
\hline Coordinate System & 13 \\
\hline Coverage & 5 \\
\hline dBASE Table & 292 \\
\hline ERDAS 7.5 LAN : 1 band(s) & 12 \\
\hline Excel File & 136 \\
\hline Excel Table & 373 \\
\hline GIF : 1 band(s) & 1022 \\
\hline GIF : 10 band(s) & 1 \\
\hline GIF : 12 band(s) & 1 \\
\hline GIF : 44 band(s) & 1 \\
\hline GIF : 6 band(s) & 1 \\
\hline GRID : 1 band(s) & 75 \\
\hline GRID Stack 7.x : 3 band(s) & 2 \\
\hline IMAGINE Image : 1 band(s) & 17 \\
\hline IMAGINE Image : 2 band(s) & 12 \\
\hline IMAGINE Image : 3 band(s) & 1 \\
\hline IMAGINE Image : 6 band(s) & 16 \\
\hline
\end{tabular}

\begin{tabular}{|c|c|}
\hline Info Table & 19 \\
\hline Interchange File & 40 \\
\hline JFIF : 3 band(s) & 1250 \\
\hline Label Feature Class & 522 \\
\hline Layer & 8 \\
\hline Map Document & 14 \\
\hline MrSID : 1 band(s) & 70 \\
\hline MrSID : 3 band(s) & 164 \\
\hline PC Arclnfo Coverage & 1878 \\
\hline Personal Geodatabase & 8 \\
\hline Personal Geodatabase Feature Class & 339 \\
\hline Personal Geodatabase Feature Dataset & 56 \\
\hline Personal Geodatabase Raster Dataset & 5 \\
\hline Personal Geodatabase Relationship Class & 6 \\
\hline Personal Geodatabase Table & 36 \\
\hline PNG : 3 band(s) & 2 \\
\hline Point Feature Class & 575 \\
\hline Polygon Feature Class & 522 \\
\hline Raster Dataset & 77 \\
\hline Route Feature Class & 6 \\
\hline Scene Document & 3 \\
\hline Shapefile & 1321 \\
\hline Text File & 216 \\
\hline Tic Feature Class & 1883 \\
\hline TIFF : 1 band(s) & 48 \\
\hline TIFF : 3 band(s) & 38 \\
\hline TIFF : 6 band(s) & 1 \\
\hline TIN Dataset & 3 \\
\hline XML Document & 44 \\
\hline \multicolumn{2}{|l|}{ (blank) } \\
\hline Grand Total & 14835 \\
\hline
\end{tabular}

Shapefiles were sorted by feature type and by theme. For instance, all line features with file names containing English words such as "highways," "HWY" and "roads" were sorted together. Similarly, features with file names containing Spanish words such as “vias," "bulevares,” and “carreteras” (Spanish term for roads, boulevards, and highways, respectively) were sorted together and compared to each other for accuracy and age. Date last modified was compared to determine the age of data. The oldest road layers were last modified in 1998. The most current data was modified in 2006. Metadata for digital data were non-existent.

Problems arose because of duplicated datasets for all base layers. Further problems arose due to frequent multiple versions of the same data. Base layers that had been modified were not apparent. Some data values were collected in Spanish and some in English. For the purpose of this project, all data remained untranslated. Spanish data can be properly translated into English or vice-versa at a later date.

It was also discovered that no serious efforts had been made to develop a standardized database for the existing spatial data, which vary widely in scale, projection, content, theme, date, and format. This made it extremely difficult to determine the spatial and attribute accuracy of the data layers.

Due to time constraints, it was decided that the client would select the base layers. Vector layers included the protected areas boundary, national roads (Figure 4-1), regional roads (Figure 4-2), national rivers (Figure 4-3), regional rivers (Figure 4-4), forestry 
(Figure 4-5), ecosystem (Figure 4-6), soils (Figure 4-7), and life zone (Figure 4-8). Raster layers included the scanned topo image (Figure 4-9), satellite image (Figure 4-10), orthographic photography (Figure 4-18) and digital elevation model (DEM) (Figure 411). All the base layers were considered to be adequate and appropriate for the objectives of this prototype web GIS application. The key assumption here is that the base layers will be replaced through maintenance with improved data management and data quality control measures at a later date. 


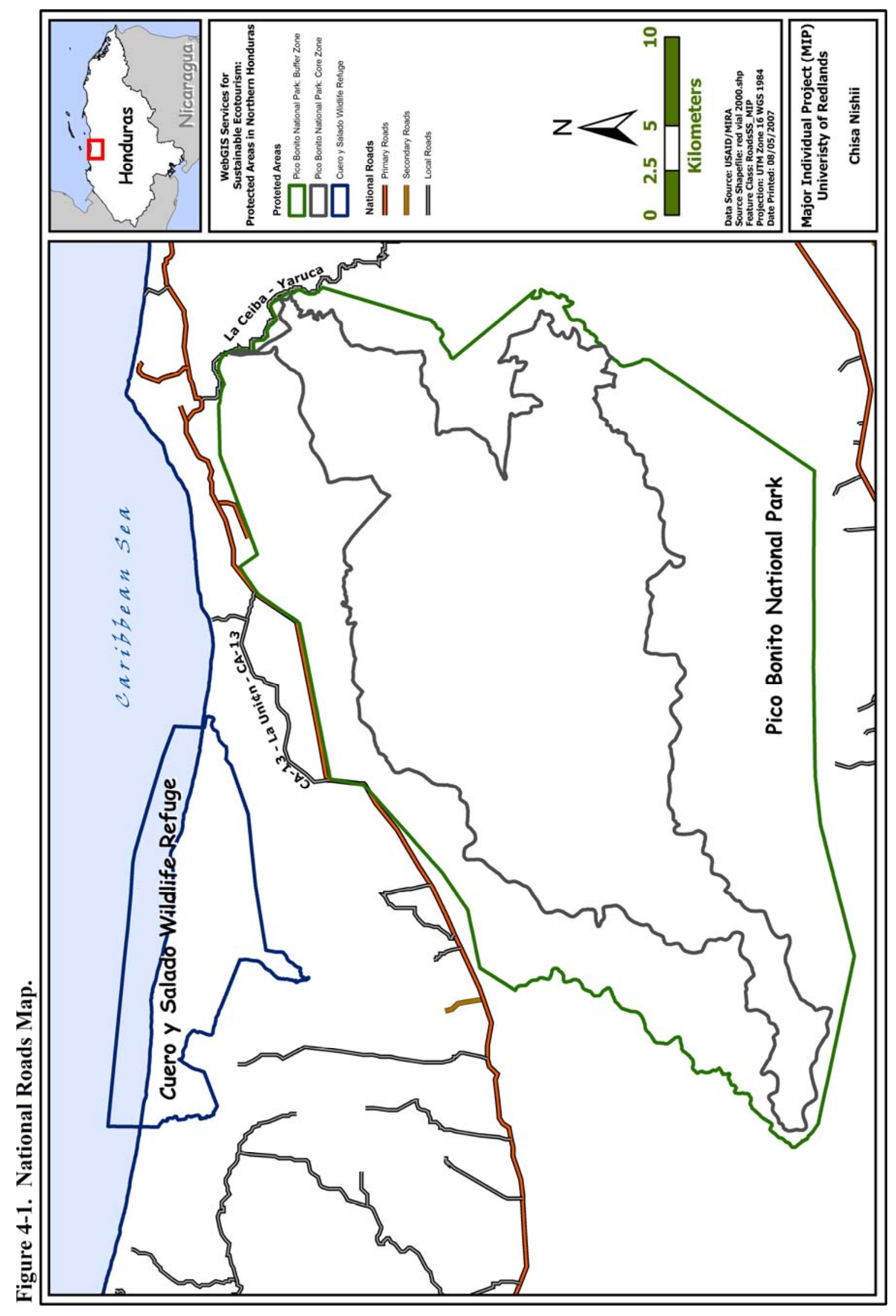




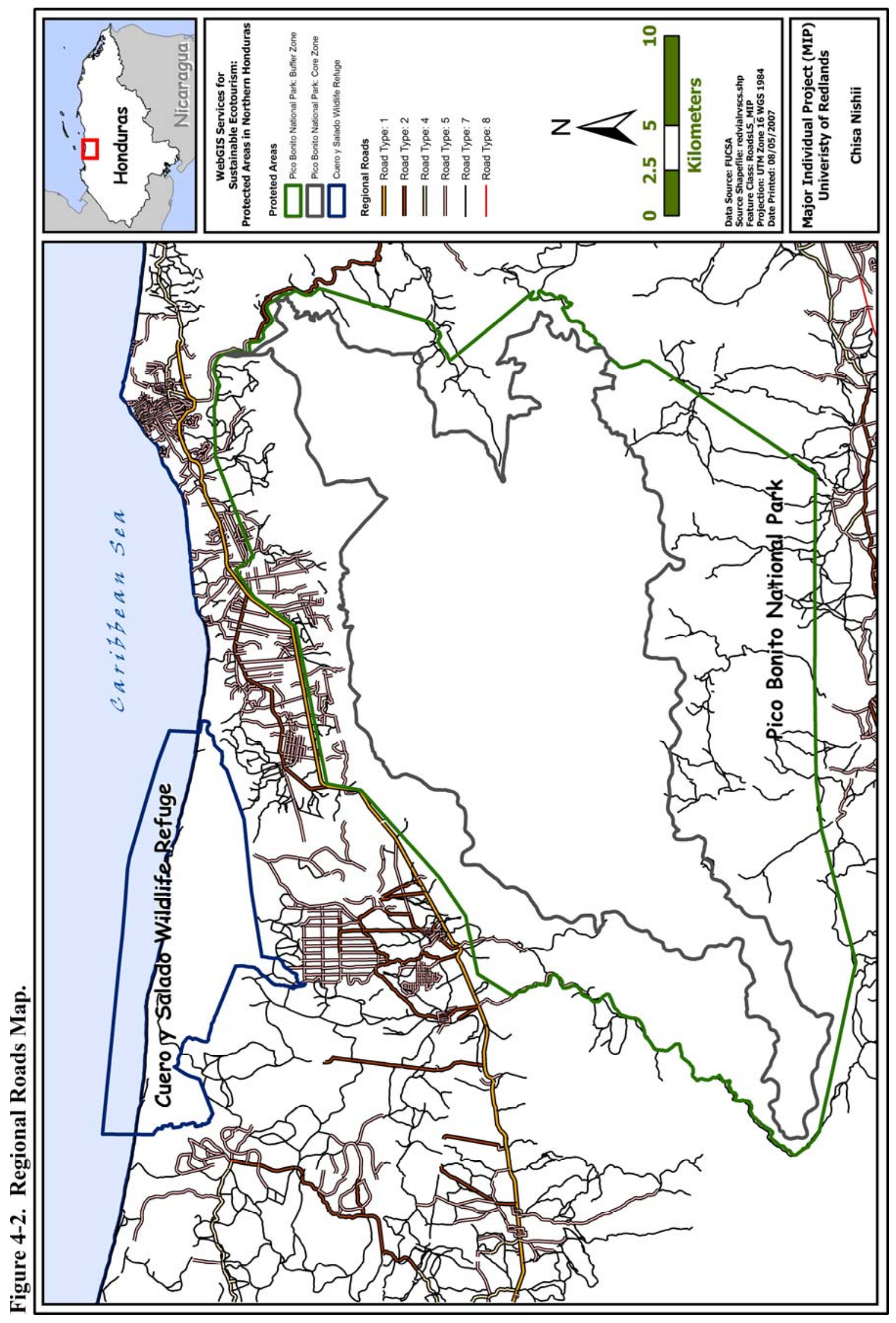




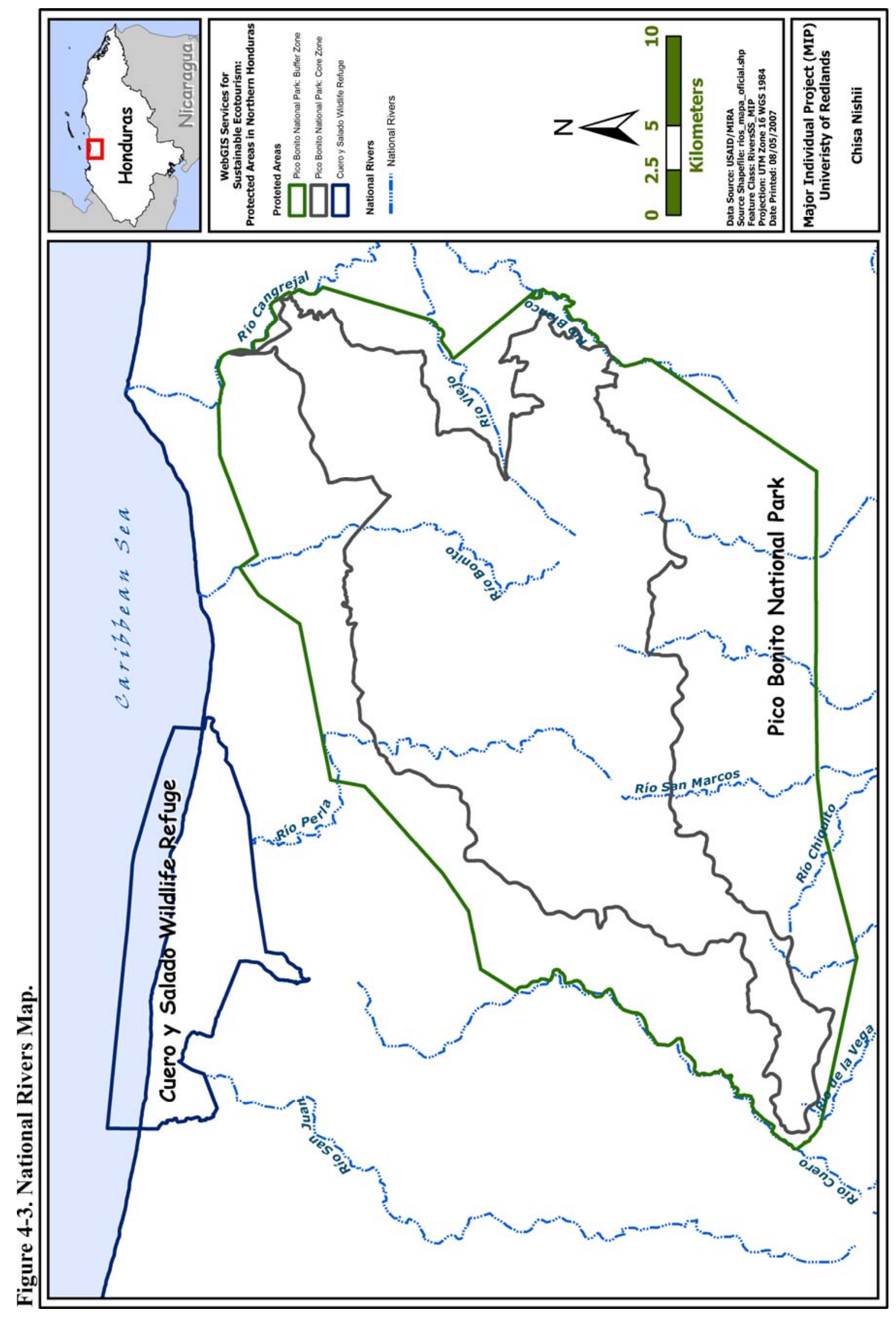




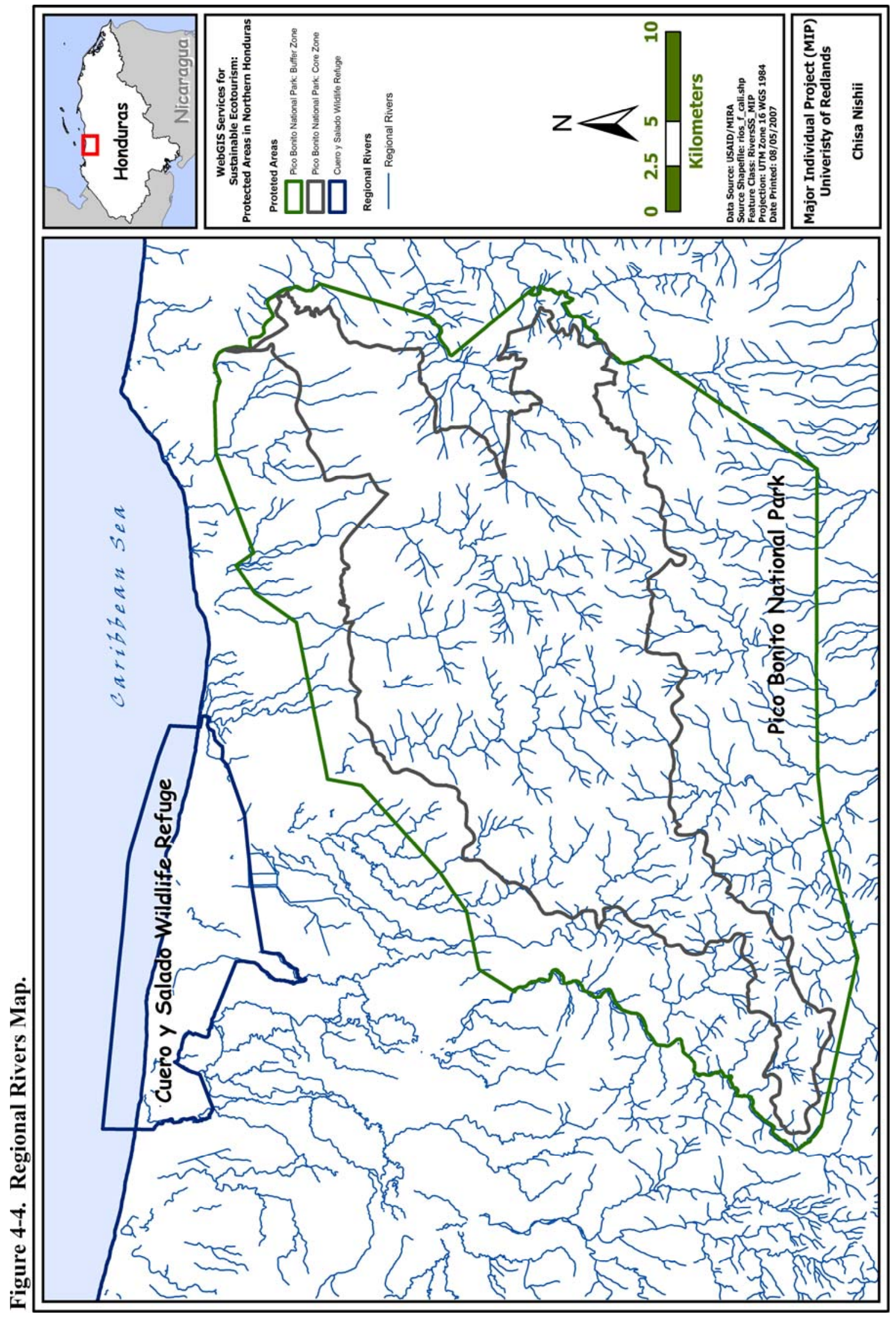




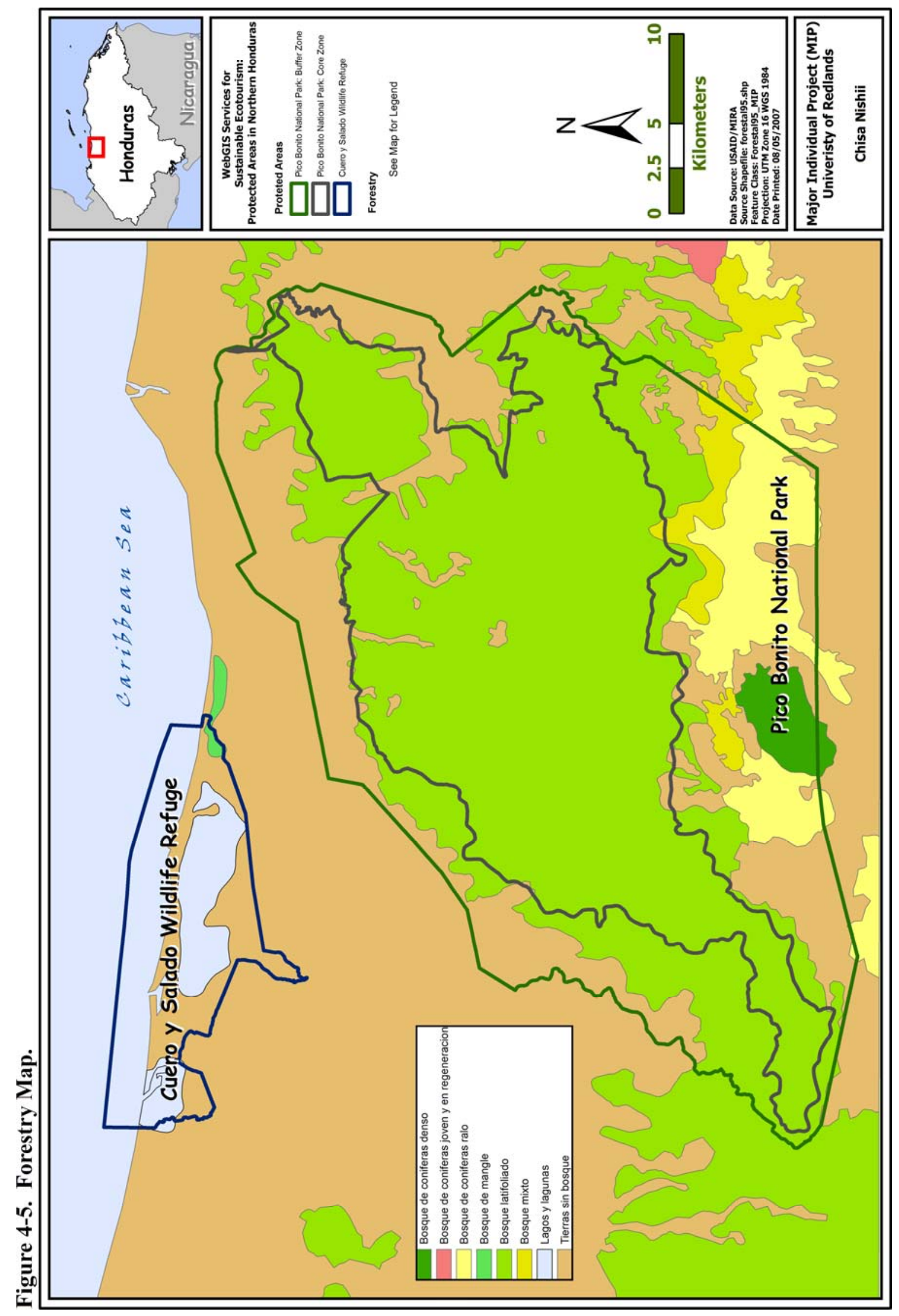




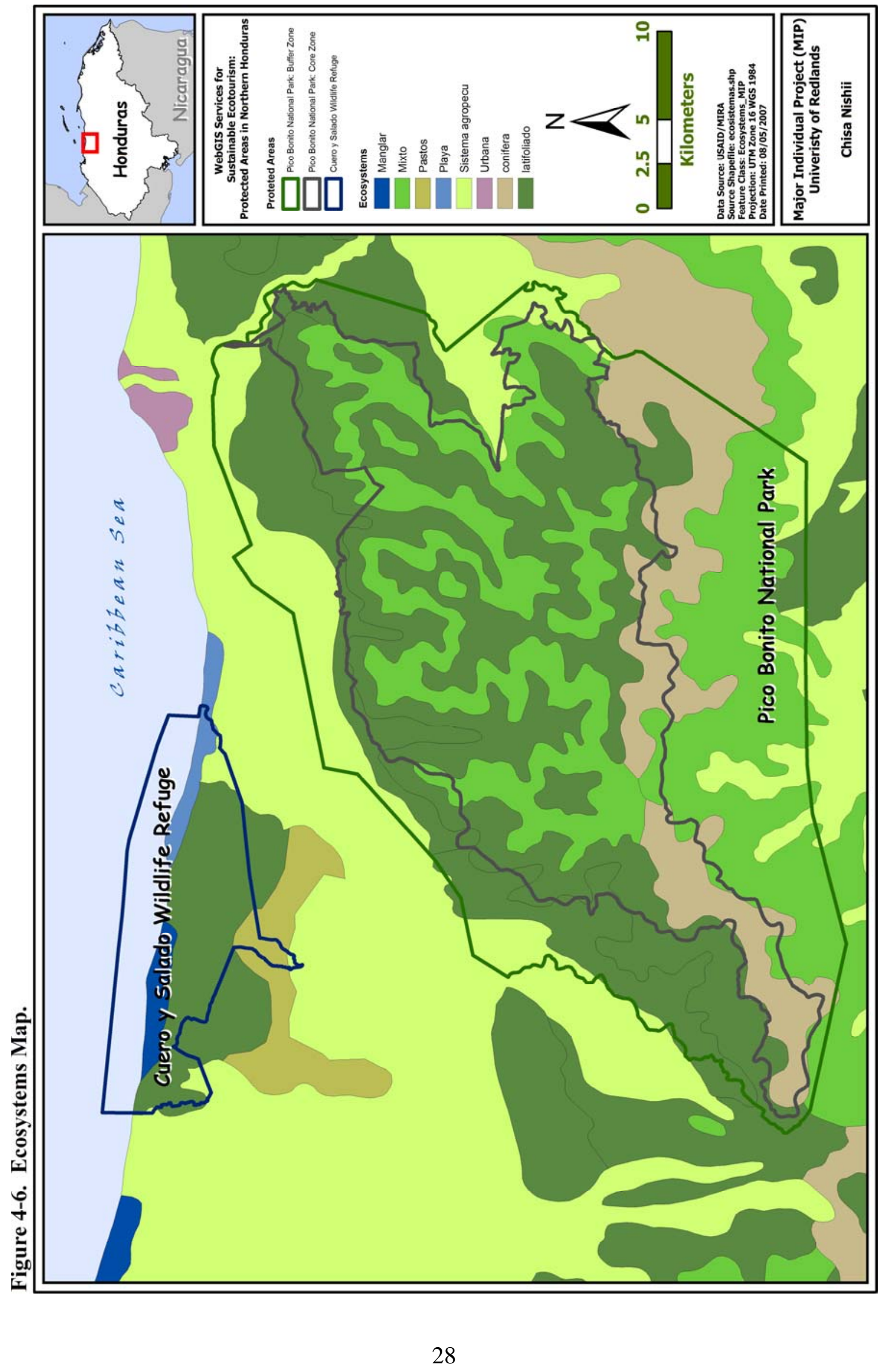




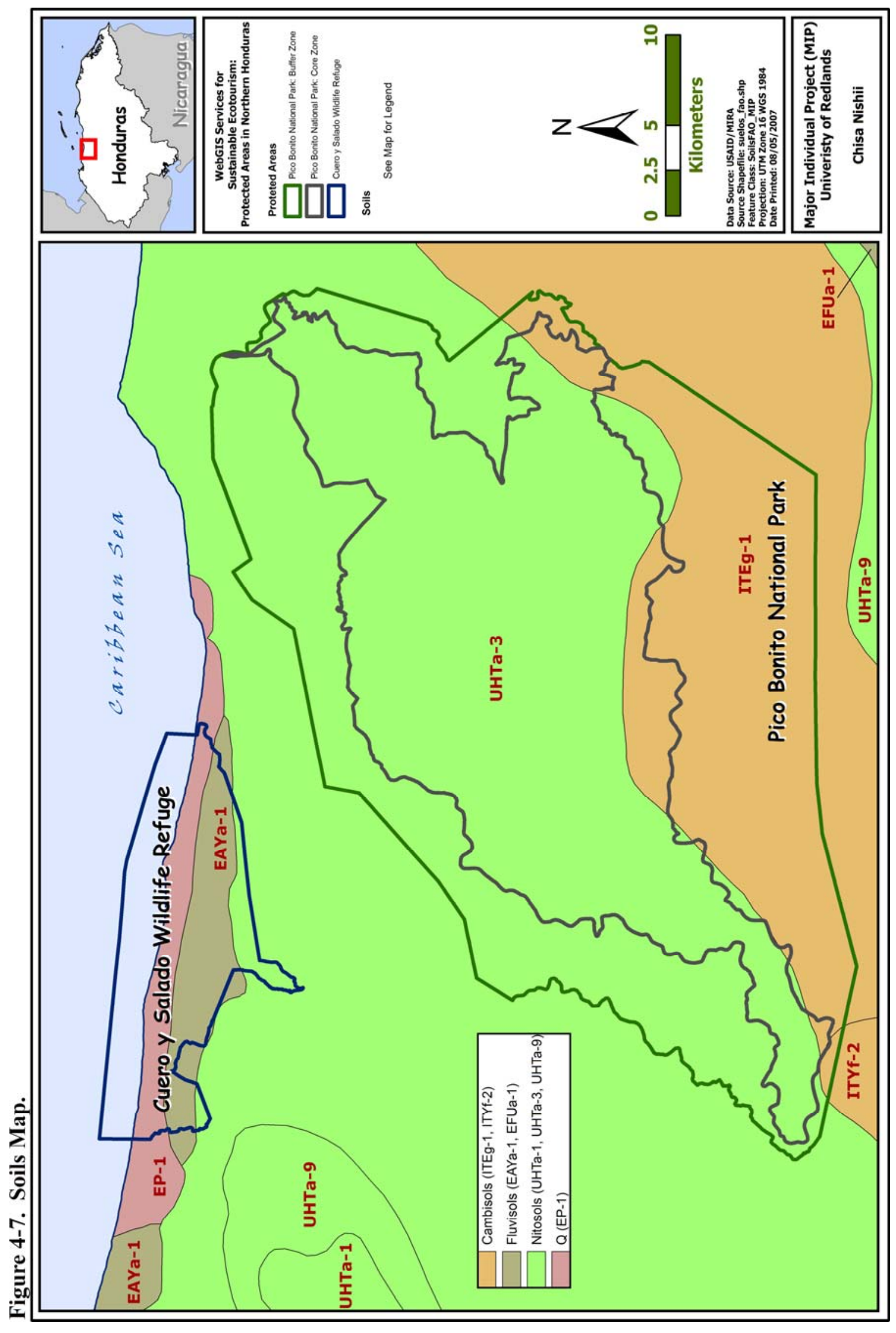




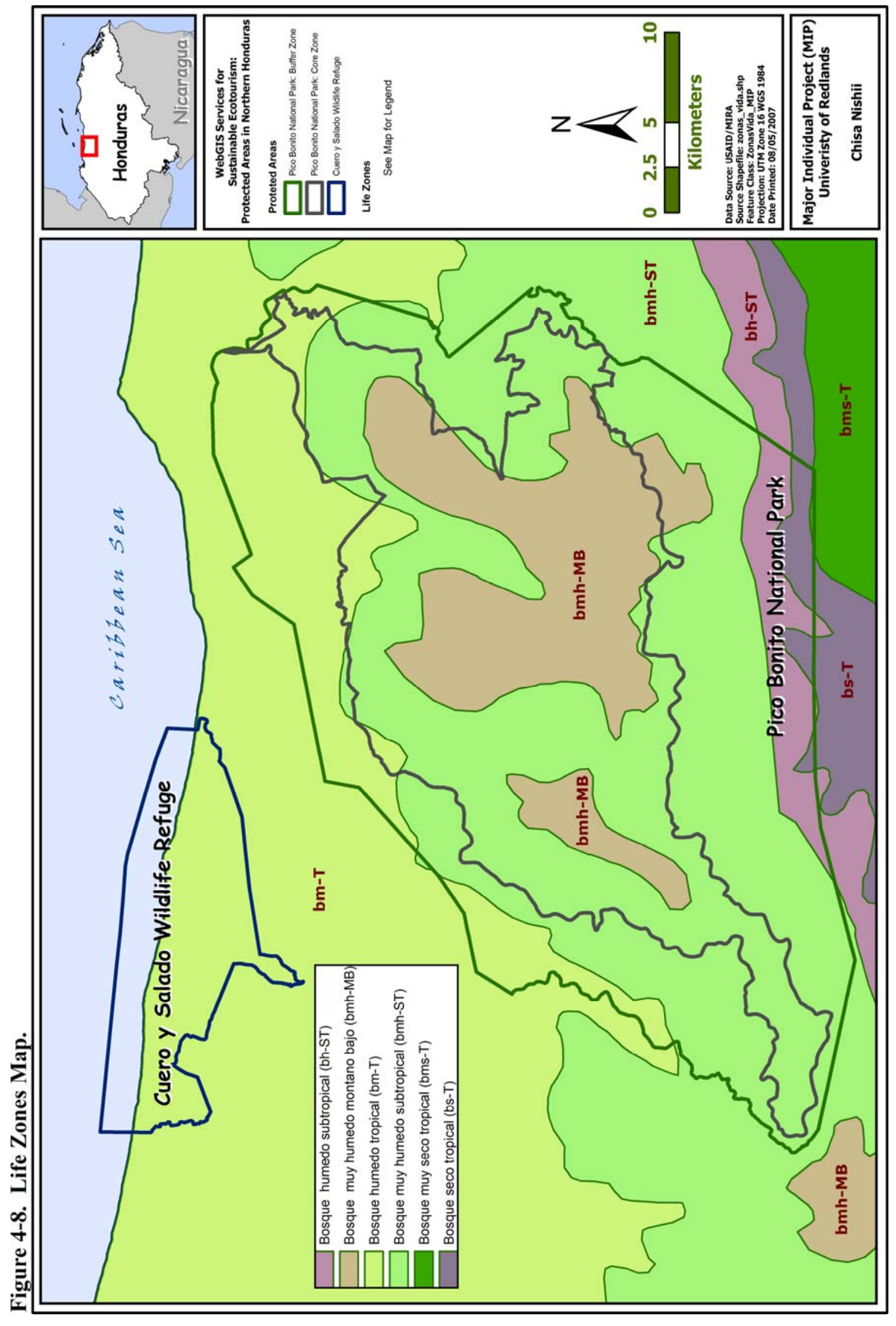




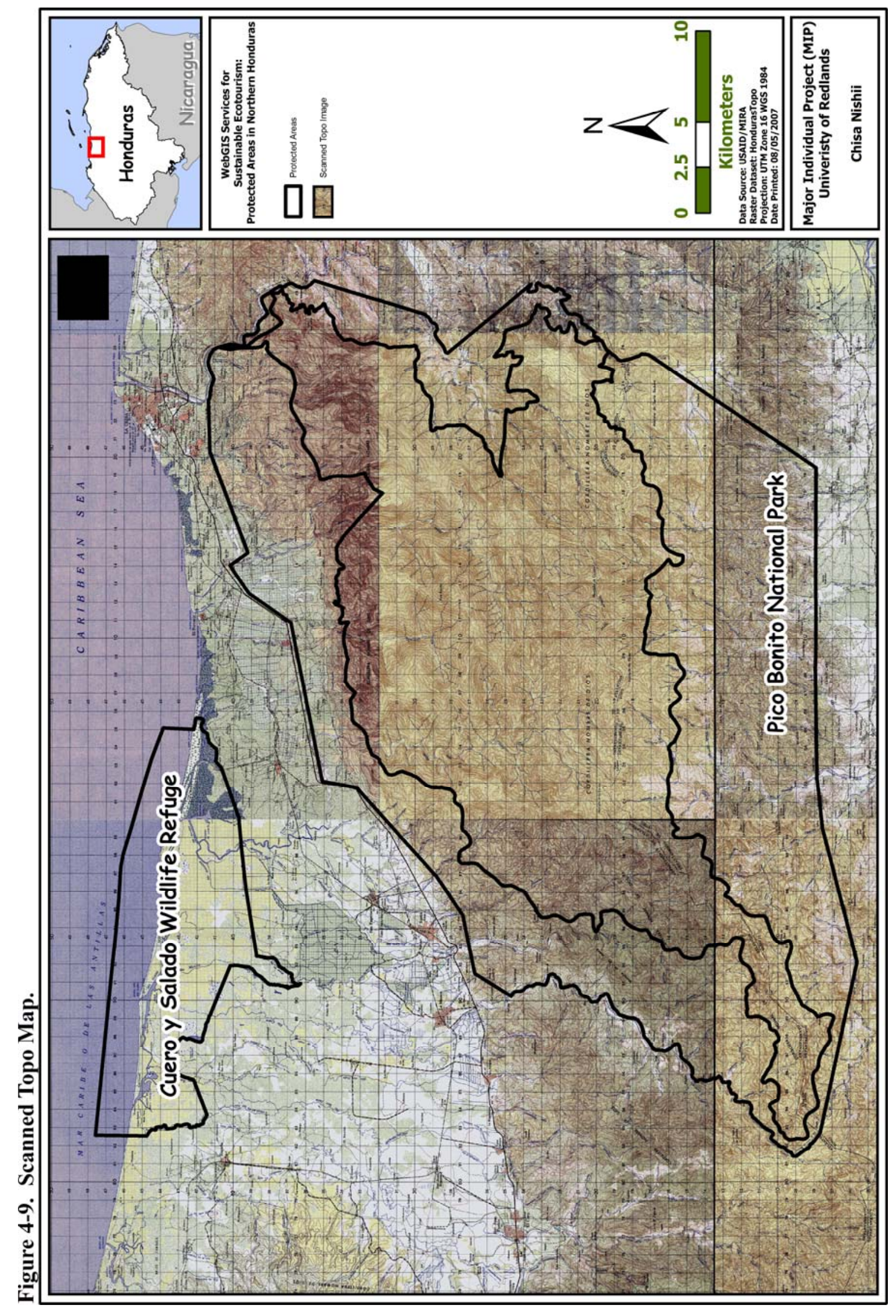




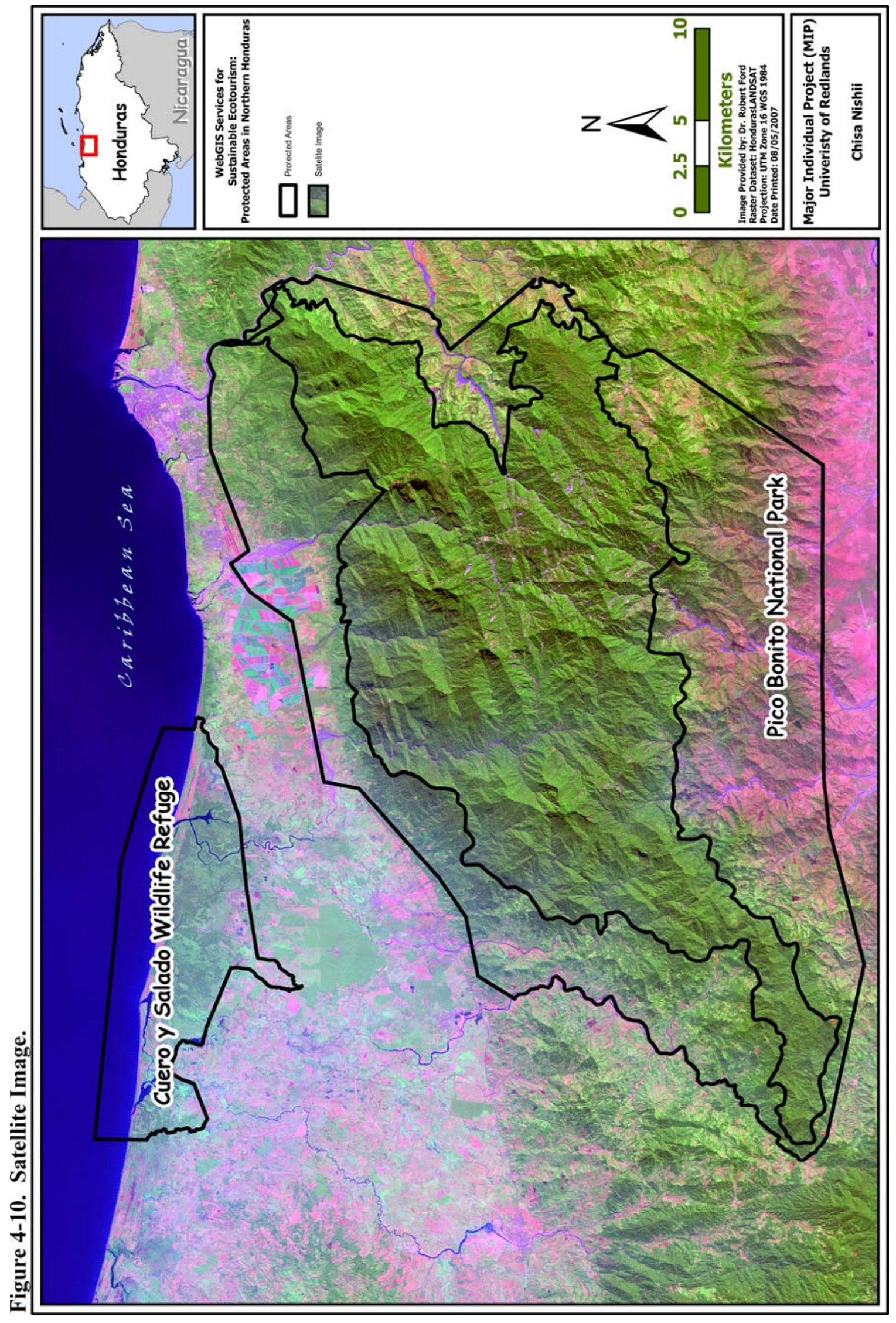




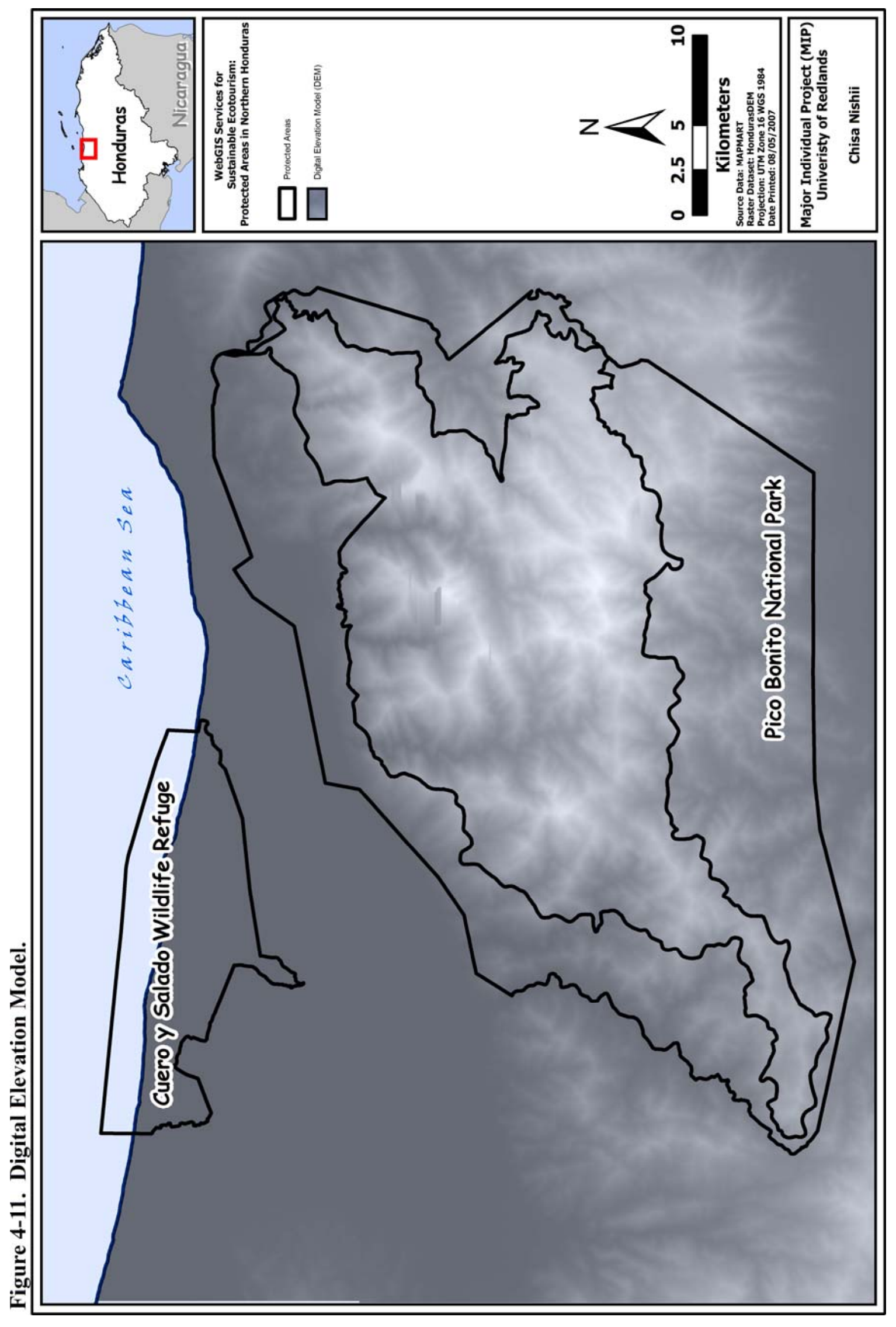




\subsection{Field Data Collection}

Initially, there was a lack of spatial data specific to the two protected areas: Pico Bonito National Park and Cuero y Salado Wildlife Refuge. Tourists are interested in available hiking trails, tours, activities and events, locations of facilities, weather condition, and bird watching sites in the protected areas. Unfortunately, there are no reasonable trail maps or tourist maps available at the park. There is also a lack of locational data, such as lodging and dining information within and around the protected areas.

For the purpose of this project, three kinds of GPS data were newly collected in Honduras: ecotourism services, trails, and photo points. Field data was collected in UTM Zone 16 projection and WGS 1984 datum.

\subsubsection{Ecotourism Services}

A variety of services are offered for tourists around the project site. GPS data for ecotourism service locations were collected by field assistants in Honduras using handheld GPS units. Table 4-4 contains the complete list of data types captured in the field, as specifically defined by the client.

Table 4-4. List of Ecotourism Service Location Types

\begin{tabular}{|c|c|c|}
\hline - Airline Office & - Environmental Agency & - Mall / Megaplaza \\
\hline - Airports & - Fast Food & - Market \\
\hline - Arts \& Crafts & - Ferry Station & - Mon \& Pop Stores \\
\hline - ATM & - First Aid Station & - Natural History Collections \\
\hline - Auto Rental & - Food Court & - NGO Office \\
\hline - Bakery & - Gas Station & - Park \& Refuge Visitor Center \\
\hline - Banking & - Government Agency & - Pharmacy \\
\hline - Bar & - Harbor / Marina & - Plant Nursery \\
\hline - Bookstore & - High School & - Primary Schools \\
\hline - Botanical Garden & - Hospital & - Public Market \\
\hline - Cafeteria & - Hostels & - Railroad Office \\
\hline - Camping Site & - Hotels & - Regional Bus \\
\hline - Health Center & - Inns (B\&B) & - Research Center/Lab \\
\hline - Club & - International Tour Operator & - Restaurant \\
\hline - Commercial Tour Operator & - Internet Café & - Supermarket \\
\hline - Community Center & - Information Kiosk & - Taxi Stand \\
\hline - Courier Service & - Language School & - Telephone Call Centers \\
\hline - Cultural Center & - Laundromat & - Tourism Agency \\
\hline - Dental Office & - Liquor Store & - Travel Agency \\
\hline - Department Store & - Local Bus & - University \\
\hline - Doctor's Office & - Local Eateries & \\
\hline - Ecolodge & - Community-based Tour Operator & \\
\hline
\end{tabular}


Approximately 200 GPS points were captured (Figure 4-12), and pictures were taken to provide attribute information for each location. Attribute information also included place name and description of the location to provide tourists with basic information about the place, and to assist them in determining where to visit, stay overnight, or dine. The attribute information was entered in a separate Excel spreadsheet by the client (refer to EcotourismServicePoints_REV7.xls enclosed on the project CD).

\subsubsection{Photo Points}

There are multiple points of interests recommended by the park and refuge staff for visitation, especially along the trails. These include photo points of spectacular scenery, and rare plant or bird species generally observed in the vicinity of the photo point location (Figure 4-12). Data collected for this dataset was limited to GPS point data, photographs, and a description. 


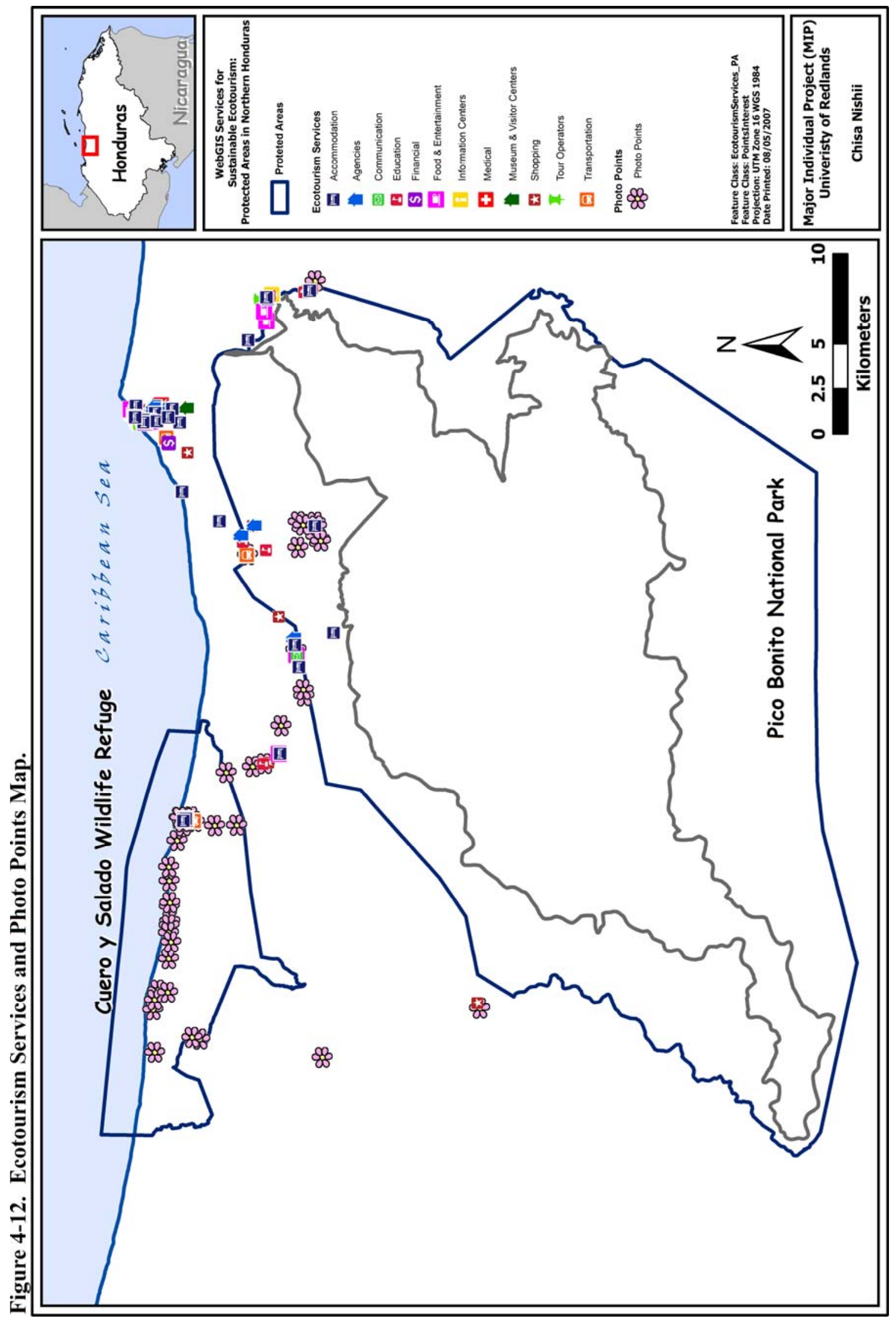




\subsubsection{Trails and Access Roads}

There are various aquatic and terrestrial trails, and access roads within and around the protected areas. A total of 15 trails and six access roads were delineated by field data collectors in Honduras using hand-held GPS units (Figure 4-13). One shapefile per line feature was provided by the client. The attribute information such as the name (e.g., Sendero Guaruma and Boca Cerrada) and type (e.g., terrestrial and aquatic) of the trail and access road features were derived from the shapefile names. Additional attribute information such as difficulty (e.g., easy, medium and difficult) was arbitrarily created by the author for web-GIS application development purposes only. 


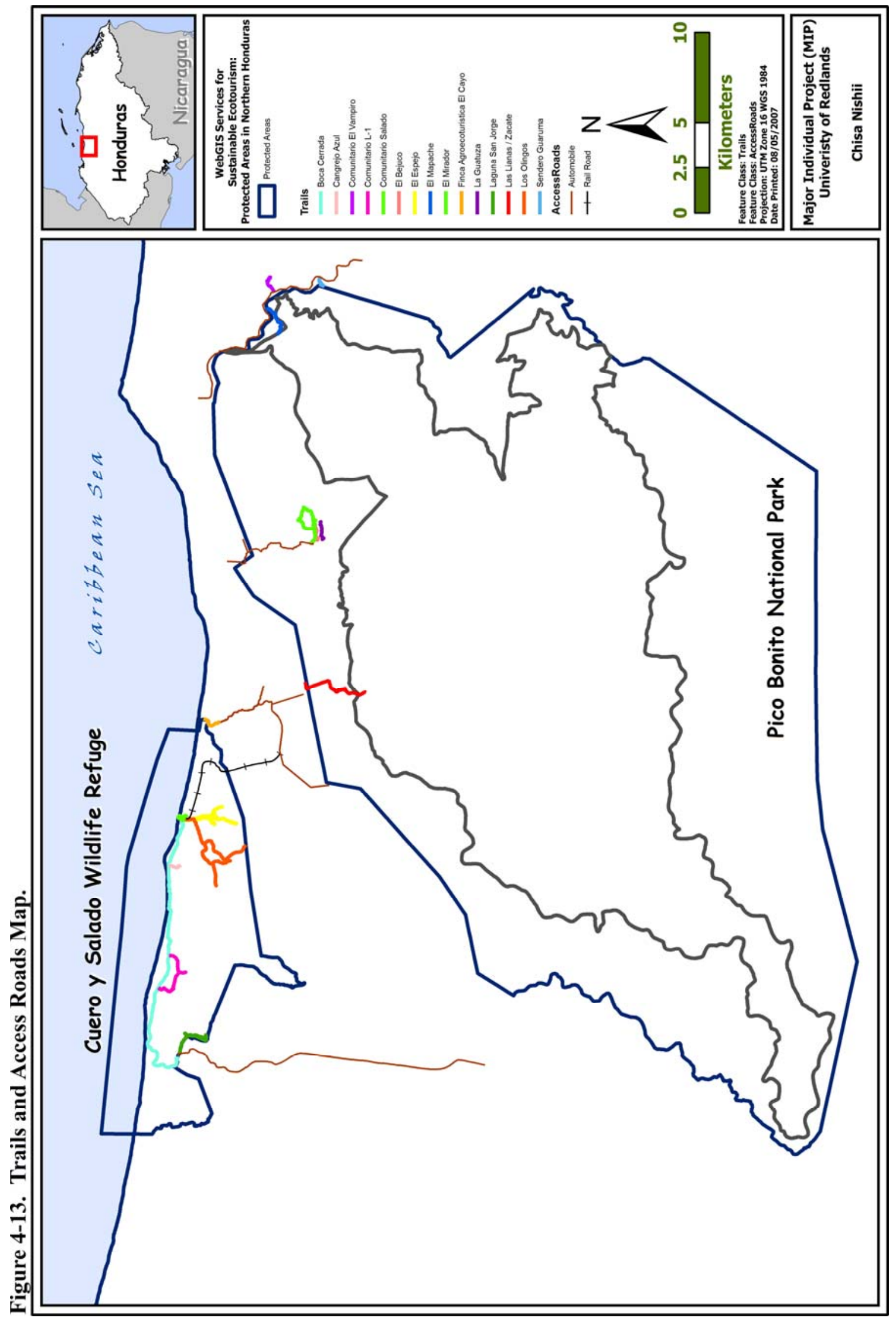




\subsection{Data Digitizing}

Existing data was supplemented with spatial and attribute data acquired from books and Internet sources.

\subsubsection{Project Extent}

The project extent is the rectangular area that covers the two protected areas including the city of La Ceiba. The project extent was defined by the client and heads-up digitized.

\subsubsection{Additional Ecotourism Services}

"How expensive is this hotel?" and "How expensive is this restaurant?" are common questions that tourists ask. In order to make lodging and dining pricing information available for the tourists, and allow them to select features of the same price range, attribute data on pricing was derived from two travel guide books (Figure 4-14).
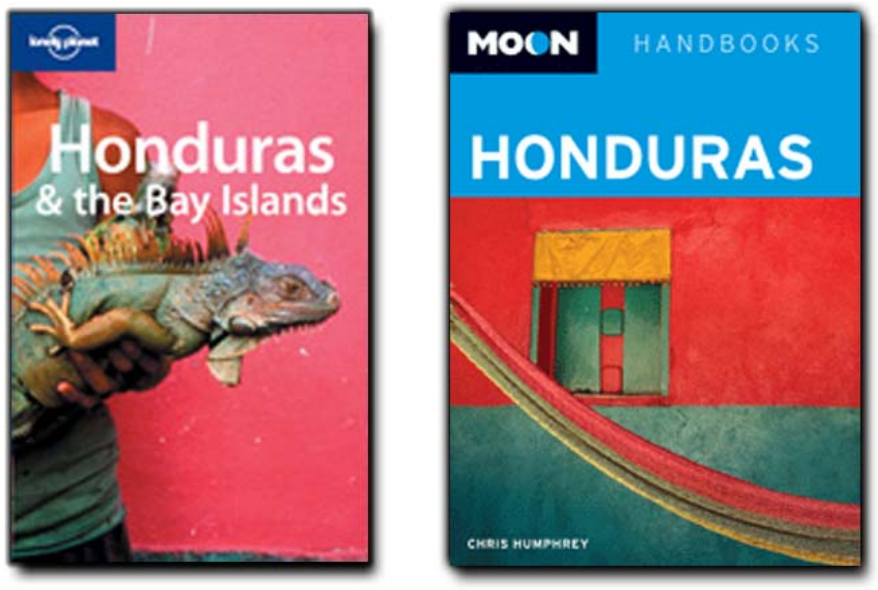

Figure 4-14. Deriving Attribute Information from Travel Guide Books. ${ }^{10}$

The Lonely Planet Travel Guide (Chandler \& Prado, 2007) uses three price categories: budget, midrange, and top-end for both lodging and dining facilities. Moon Handbooks (Humphrey, 2006), on the other hand, uses a more specific price category for lodging facilities: under US\$10, US \$10-25, US \$25-50, and US \$50. Lonely Planet's price categories were utilized for the dining price information, while Moon's price categories were used for the lodging price information of the individual ecotourism services. The value Information is not available was entered as needed.

\subsubsection{La Ceiba Roads}

La Ceiba is the closest populated city to the two protected areas of interest. Ecotourism services point data is most centralized in these areas, and a good road map of the city was

\footnotetext{
${ }^{10}$ Images were retrieved from http://shop.lonelyplanet.com/Primary/Region/CENTRAL_AMERICA/PRD_PRD_1017/Honduras++the+B ay+Islands+1.jsp and http://www.moon.com/catalog2/honduras.html
} 
necessary to orient tourists. Location and name of roads were derived from an existing city tour map downloaded from the Internet ${ }^{11}$ (Figure 4-15).

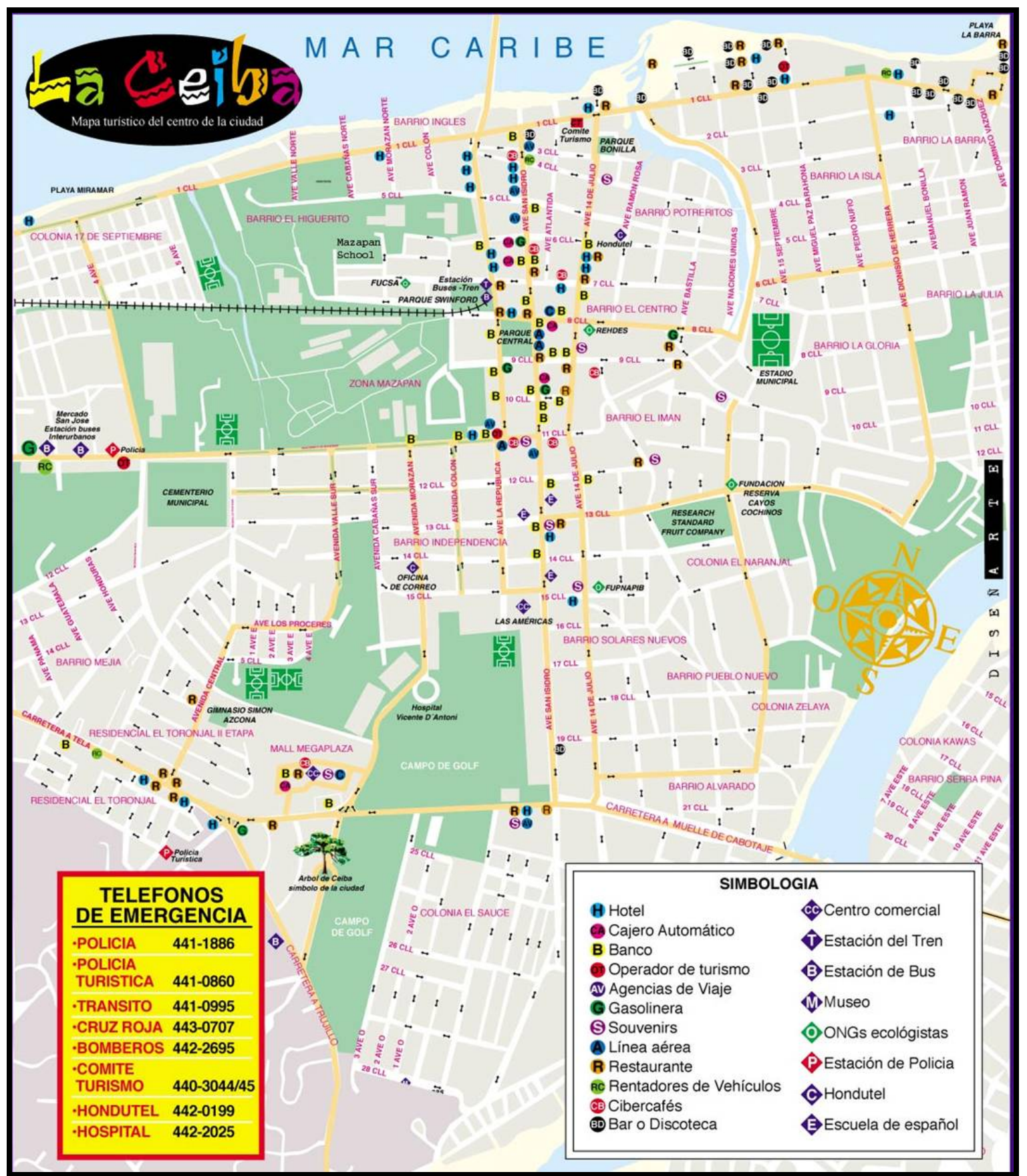

Figure 4-15. La Ceiba City Tour Map.

Two background layers were used as reference: lot lines (Figure 4-16) and orthophoto imagery (Figure 4-17), which were both provided by the USAID Honduras office and projected to UTM Zone 16 WGS 1984.

${ }^{11}$ La Ceiba Tourist Guide Map was retrieved from: http://www.esf.edu/ewb/images/ceiba_cityLG.jpg 


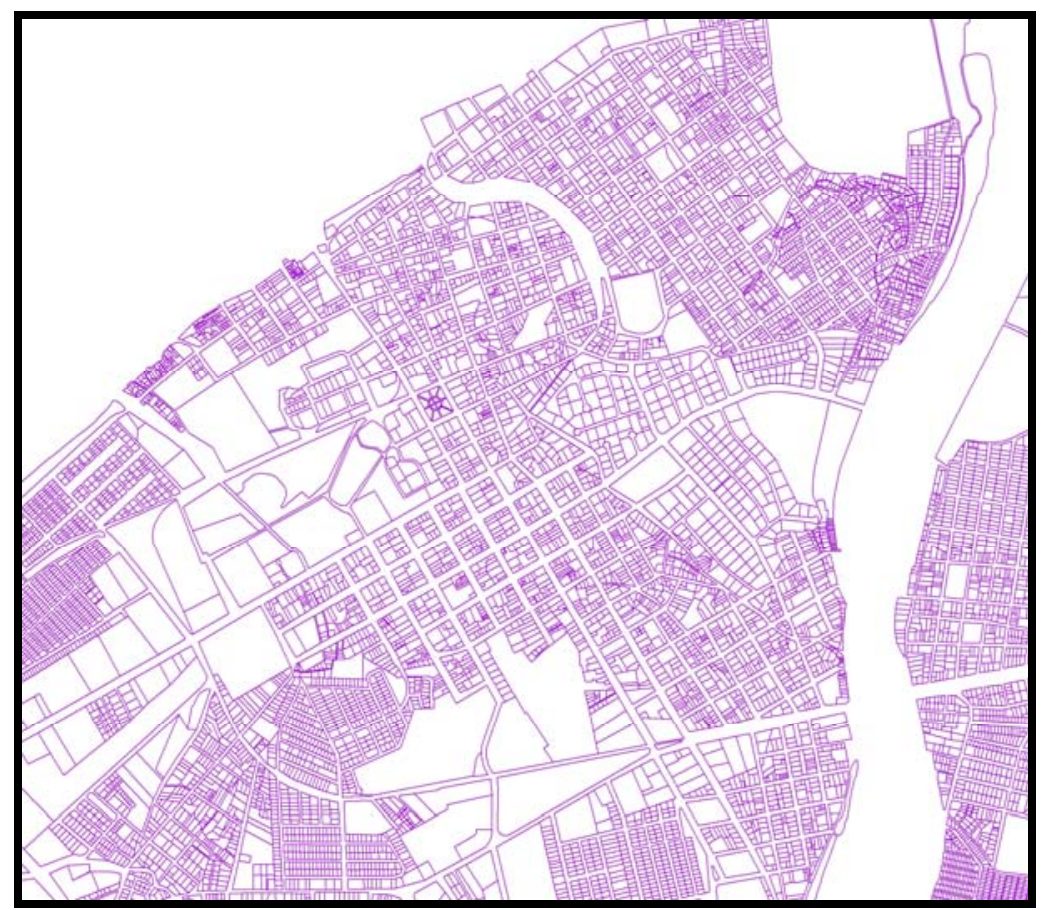

Figure 4-16. La Ceiba Lot Lines.

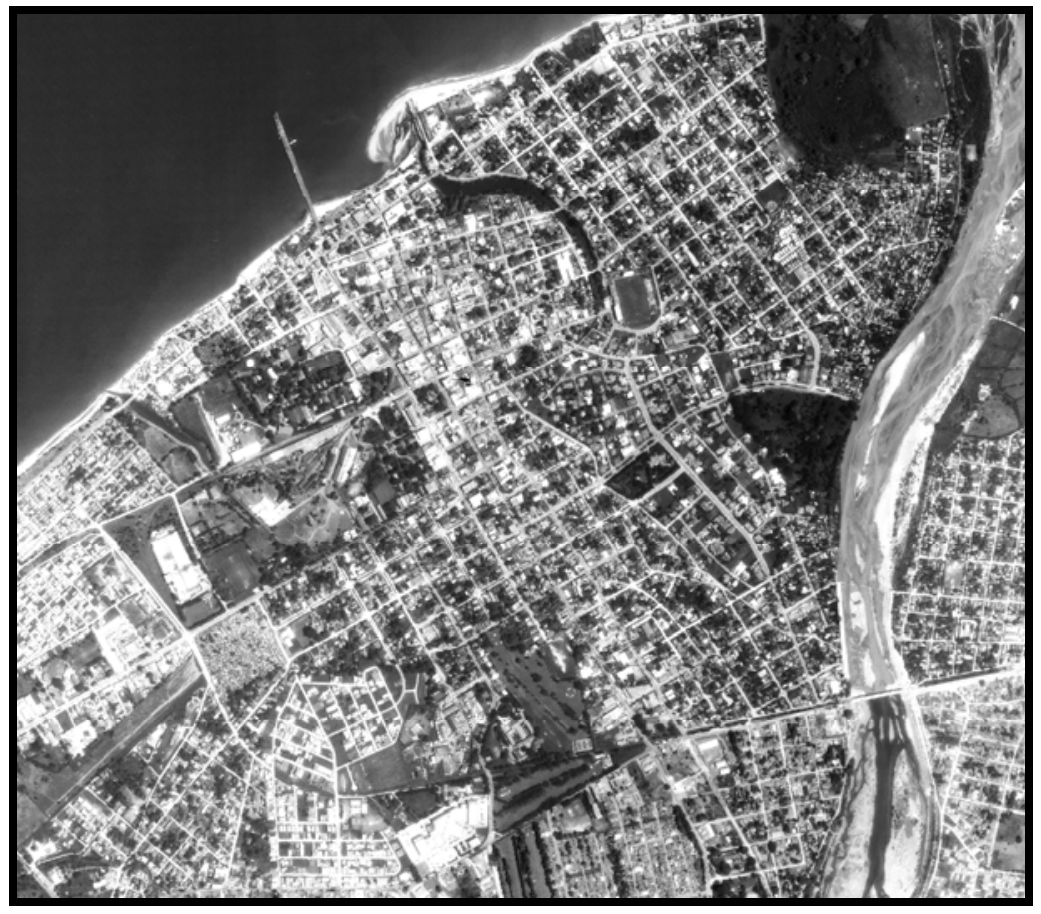

Figure 4-17. La Ceiba Orthophoto Imagery.

First, roads were digitized on the computer screen by following the centerlines of the existing lots as indicated on the tourist map. Then attribute data, such as road names from the tourist guide map, were typed into the attribute table. A total of 55 road segments were digitized, with road names associated with each segment (Figure 4-18). 


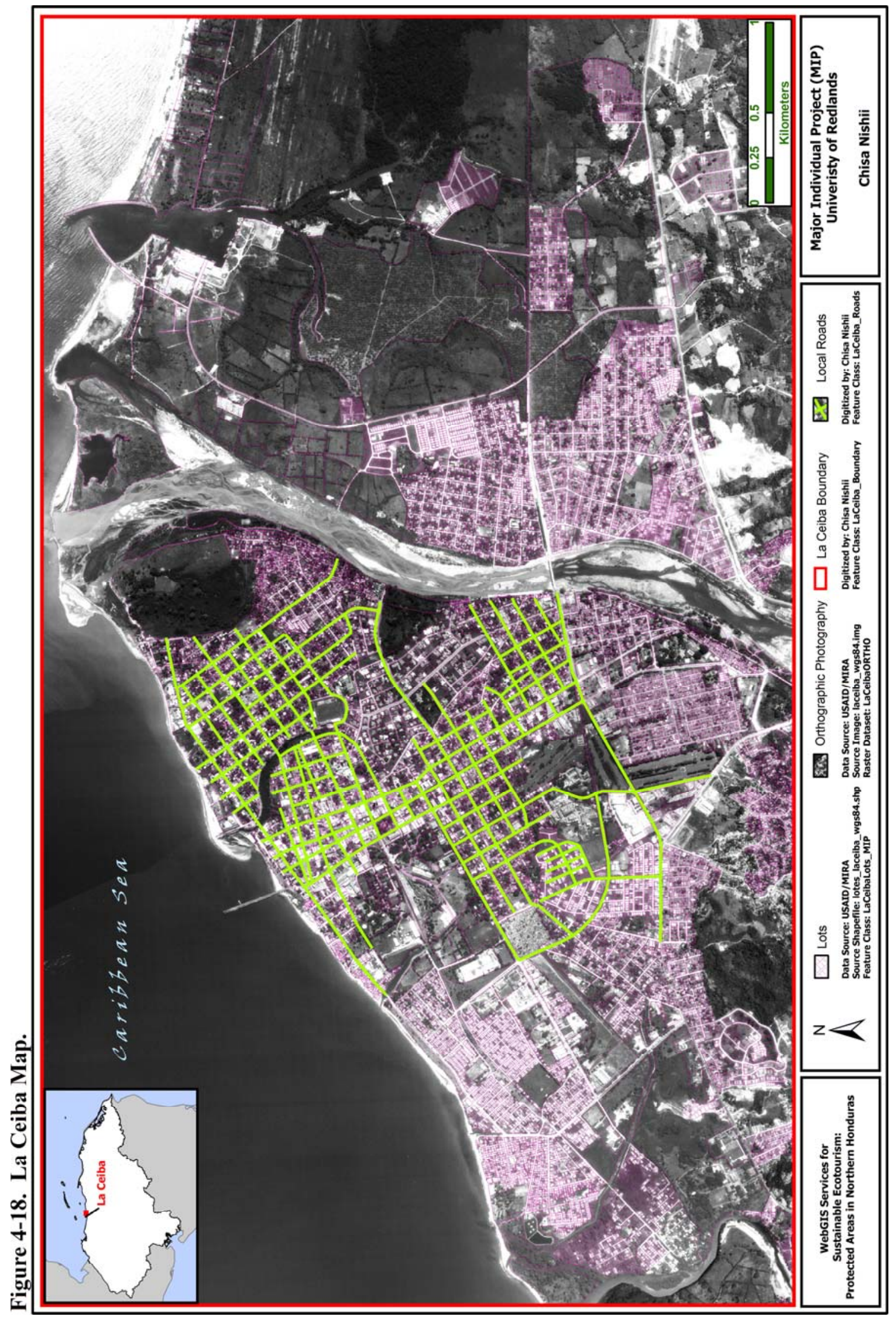




\subsection{Image Processing}

Two models were used to create the background hillshade surface for this project: Swiss model and Multidirectional Oblique Weighting (MDOW) hillshade model. The combination of two hillshades was used because of its aesthetic nature and the ability to visually convey the varied terrains in the project sites. Two hillshade models were downloaded from the ESRI support website ${ }^{12}$. Hillshade Tools are a combination of Spatial Analyst tools connected to one another in Model Builder.

\subsubsection{Creating the Swiss Hillshade}

The Swiss Hillshade Method was originally implemented within a GIS by the United States Geological Survey (USGS). Swiss method creates two new rasters from the input DEM: Median Filter Hillshade and Raster Calculator Hillshade (Figure 4-19).

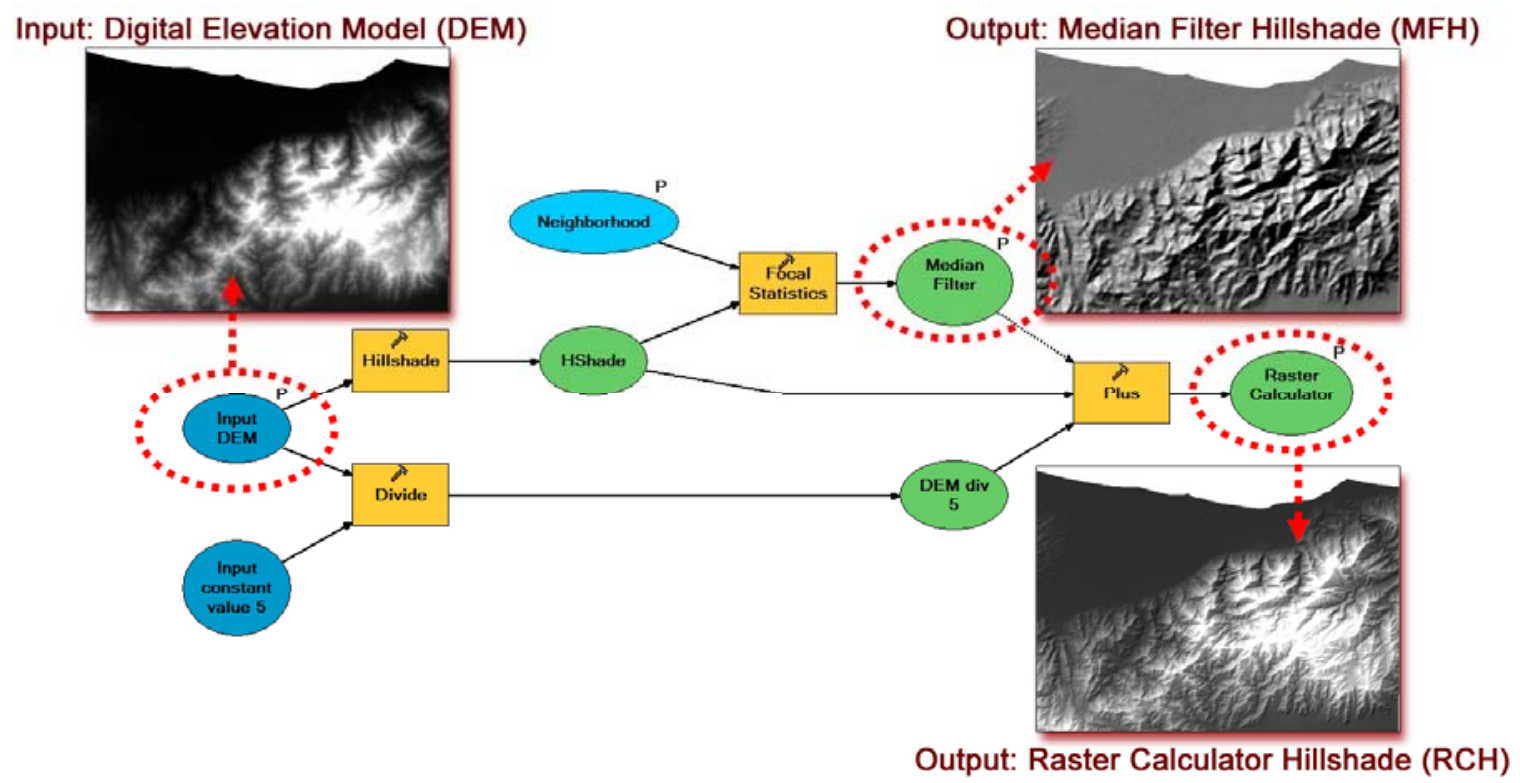

Figure 4-19. Swiss Hillshade Model.

The Median Filter produces a smoothing effect, emphasizing the major geographic features, minimizing the minor features, and smoothing irregularities on the slopes. However, it still maintains the rugged characteristics of ridge tops and canyon bottoms. The Swiss Hillshade Method "simulates an aerial perspective that makes the higher elevations lighter and the lower elevations darker.” (ESRI, 2005).

\footnotetext{
${ }^{12}$ The Hillshade Models were retrieved from: http://support.esri.com/index.cfm?fa=downloads.dataModels.filteredGateway\&dmid=3
} 


\subsubsection{Creating the Multidirectional Oblique Weighting Hillshade}

MDOW Hillshade method, on the other hand, "shades the surface from four different illumination angles so you can highlight features regardless of their orientation relative to the illumination source" (ESRI, 2005). The MDOW model creates one raster from the input DEM: MDOW hillshade (Figure 4-20).
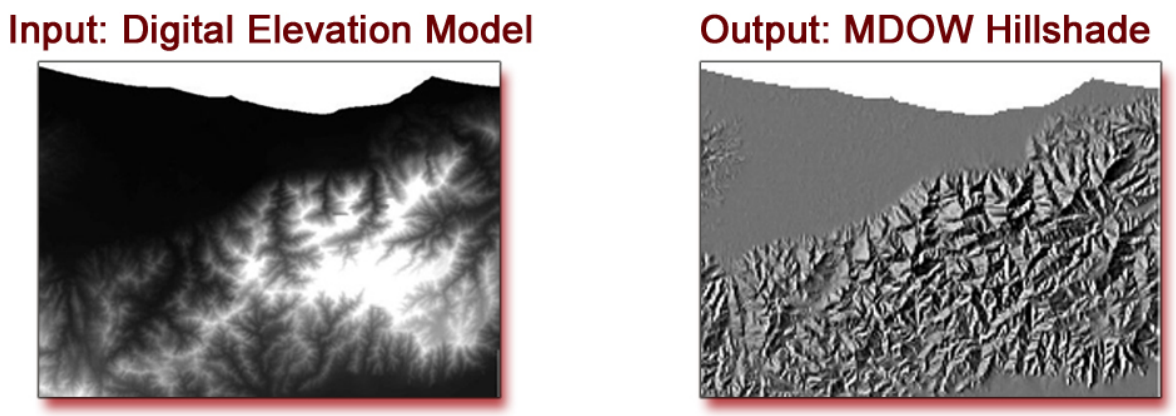

Figure 4-20. MDOW Hillshade Model Results.

\subsubsection{Combining the Two Hillshades}

When the two hillshades were overlaid in a map document, the result produces an effect similar to the Swiss Hillshade, but with additional definition in the terrain representation (Buckley, 2007). Figure 4-21 illustrates the progression of combining the various hillshades. The combination of DEM, RCH and MFH creates a Swiss hillshade (lower left). Furthermore, when the Swiss hillshade is overlaid with MDOW, the resulting hillshade adds significant amount of detail in the Swiss Hillshade (lower right).

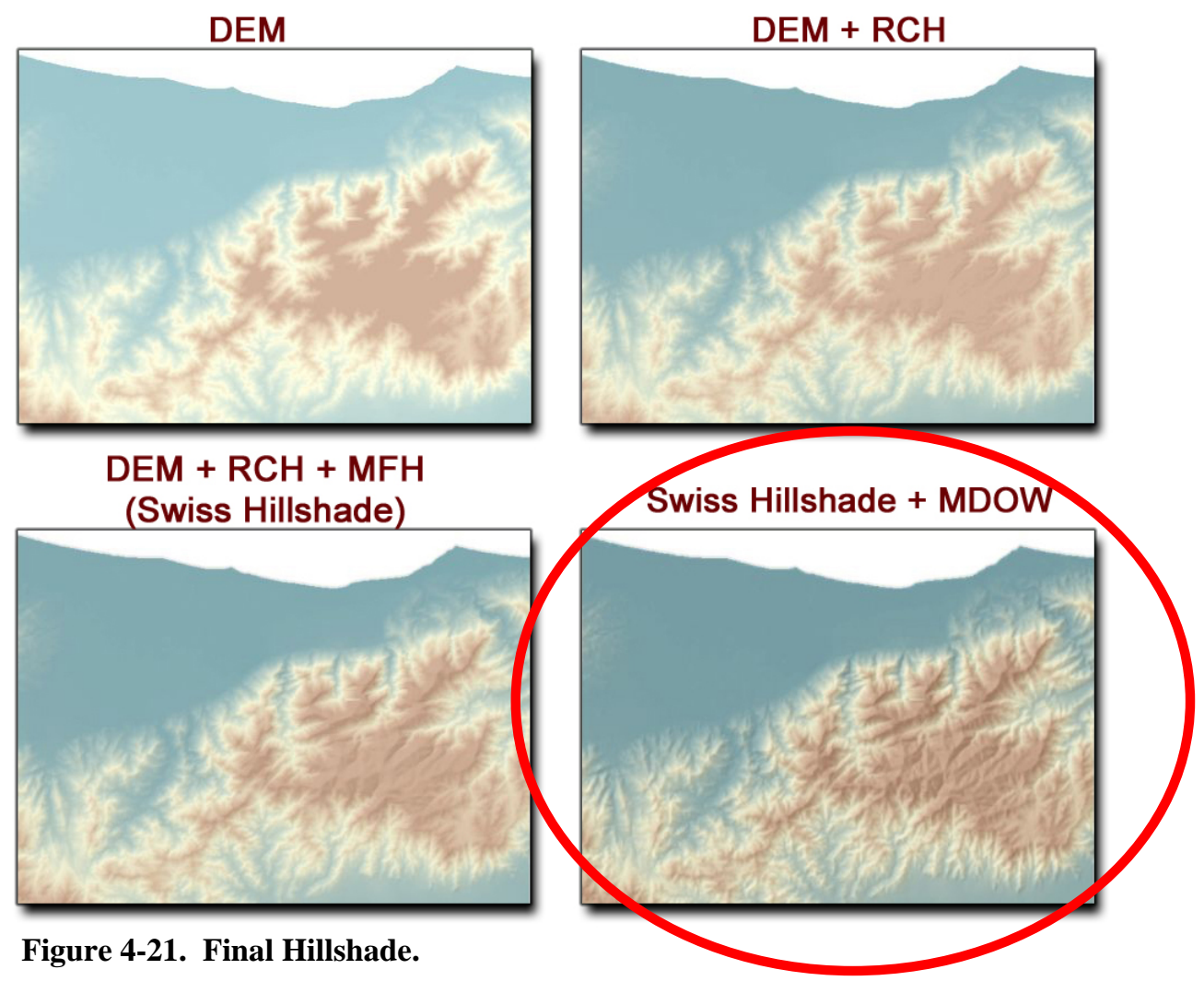




\section{Geodatabase Design}

A geodatabase is a native data structure within ArcGIS which serves as a container of spatial datasets and other elements, providing a central location to store relevant features and attributes. The geodatabase for this project was designed specifically to facilitate data entry and quick data retrieval for display. This chapter discusses the geodatabase design in more detail.

\subsection{File Geodatabase}

An ArcGIS 9.2 File Geodatabase (FGDB) was constructed as the main data repository for this project. Given the small project scale, three geodatabase types were considered and compared to one another in selecting the most appropriate geodatabase format: personal geodatabase, file geodatabase, and ArcSDE personal database. Figure 5-1 summarizes the characteristics of these geodatabase types (ESRI, 2006b).

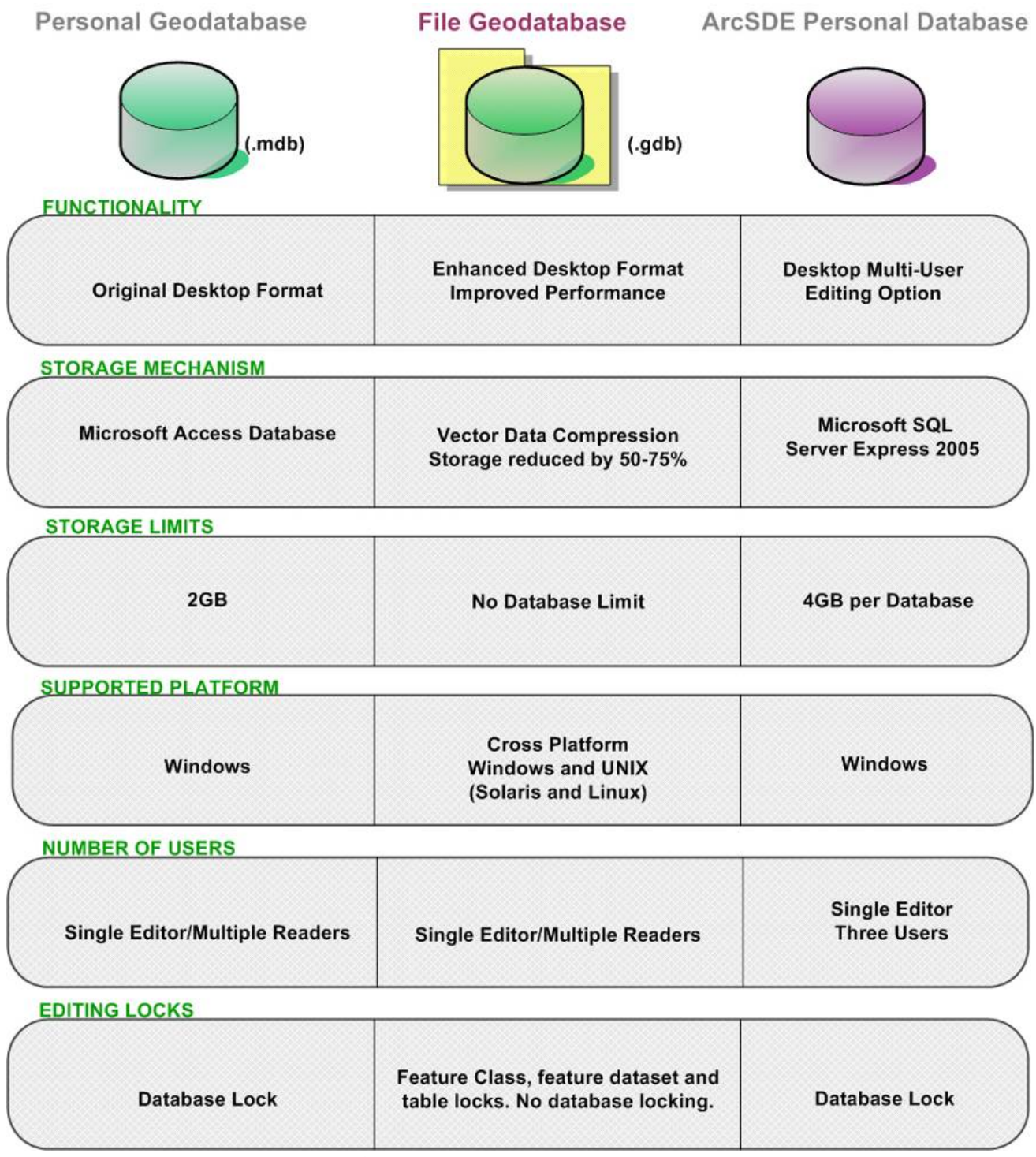

Figure 5-1. Characteristics of Geodatabase Types. 
File size was a major contributing factor in determining the most suitable geodatabase type. In comparison with the personal geodatabase and personal ArcSDE database, FGDB has a larger storage size limit and so was selected to accommodate future data expansion. It also has an efficient data storage mechanism by compressing vector data within the FGDB, which significantly reduces the database size. For example, the original data size for this project exceeded 2GB. However, it requires only 1.5GB to store the same geographic data in the FGDB. There is an option to compress the entire geodatabase to even further reduce the database size, if necessary. This unique storage mechanism results in overall improved database performance by reducing disk space, query time, and draw/redraw times.

The "SustainableEcotourism" FGDB created for this project contains seven feature datasets, 18 feature classes, and seven raster datasets (Figure 5-2). Each feature dataset contains a collection of feature classes that share a common theme and spatial reference: UTM Zone 16 WGS 1984 coordinate system, in which most datasets were initially projected. Related feature classes were grouped into feature datasets based on the base map model downloaded from the ESRI support website ${ }^{13}$.

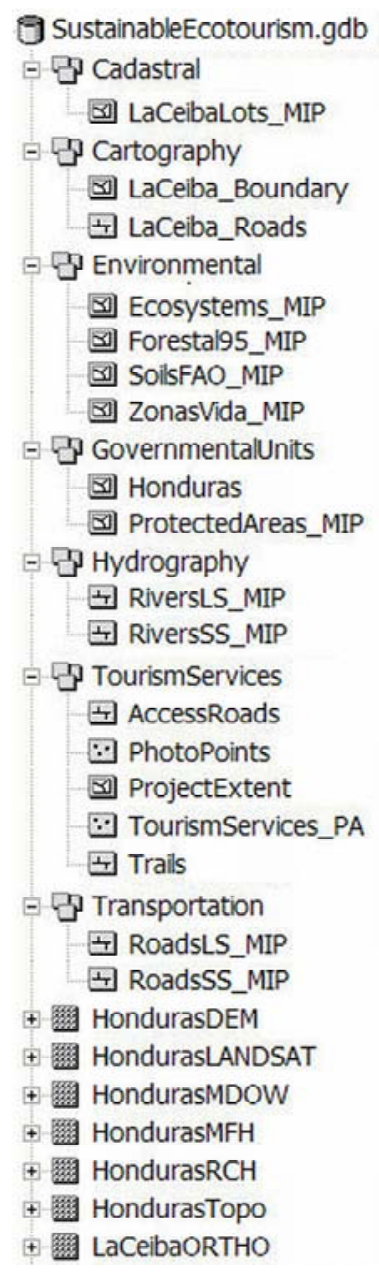

Figure 5-2. File Geodatabase Structure.

\footnotetext{
${ }^{13}$ Topographic Base Map Data Model Poster was retrieved from: http://support.esri.com/index.cfm?fa=downloads.dataModels.filteredGateway\&dmid=3
} 


\subsection{Migrating to File Geodatabase}

Migrating project data into the FGDB required both vector and raster data processing.

\subsubsection{Vector Data}

A file geodatabase stores feature classes rather than shapefiles. All existing data utilized for this project was received from the client in shapefile format, so the first step was to transfer shapefile data into feature classes. Table 5-1 lists the source shapefiles used for this project.

Table 5-1. List of Vector and Raster Data Migrated to File Geodatabase

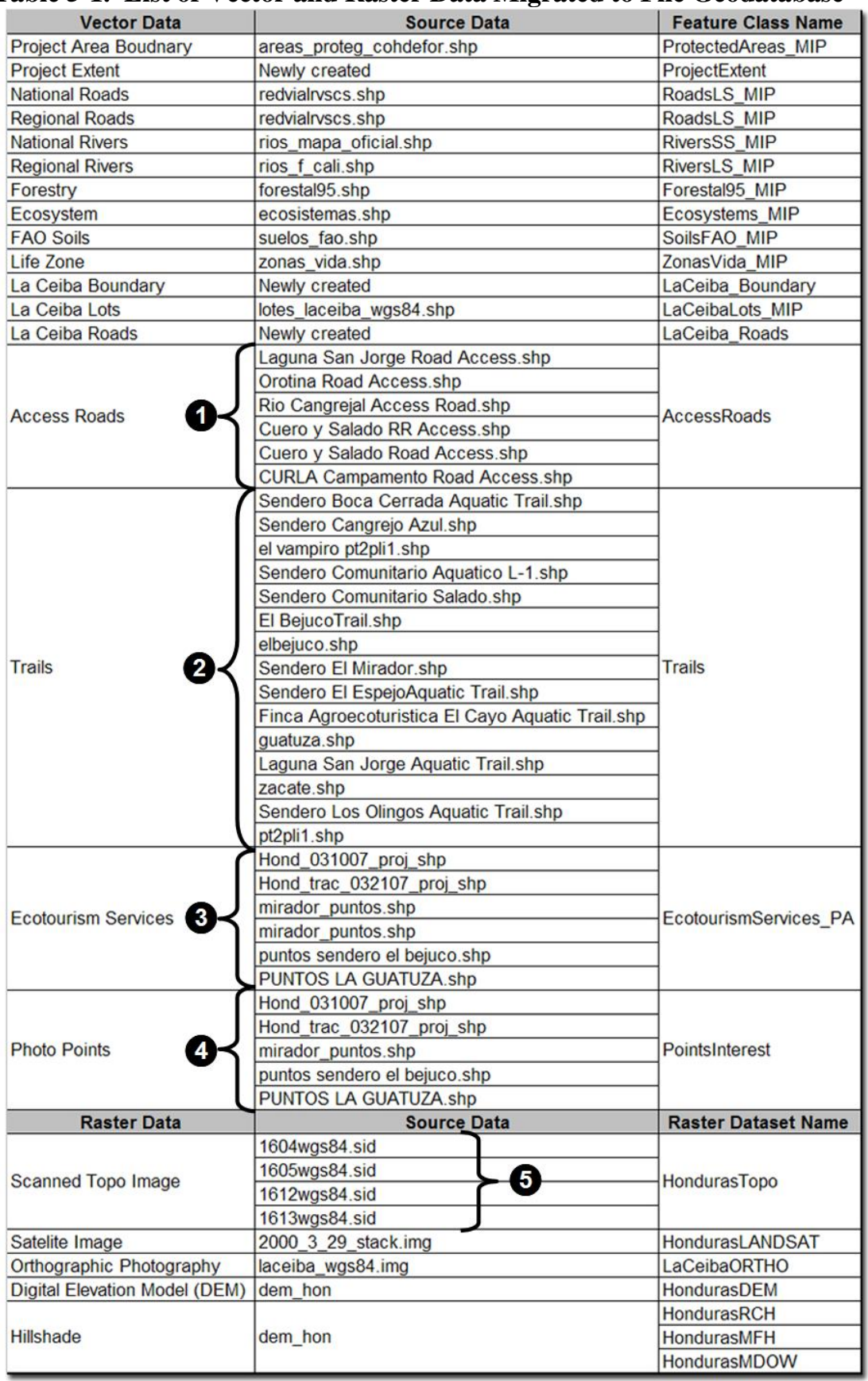


Project data from shapefiles were migrated to feature classes using the "Import Feature Class” tool in ArcCatalog. Trails, ecotourism services, access roads, and photo point data (see 1234) were received in multiple shapefiles and were merged into a single feature class using the "Load Data” tool. Attribute information for the ecotourism services point data that was entered in the Excel spreadsheet was migrated into the geodatabase using the "Join" tool in ArcMap. Newly created feature classes were then clipped to the project extent feature class using the "Clip" tool. Feature classes listed in Table 5-1 are sections of original data clipped to the project extent.

\subsubsection{Raster Data}

As shown in Table 5-1 (see (5), four separate images were provided by the client for background scanned topo image display. They were stitched together into a single raster dataset using the "Mosaic to New Raster" tool using the maximum mosaic method.

\subsection{Subtypes and Domains}

Subtypes categorize records into major groups, and domains are lists of pre-defined values for attributes. Actur \& Zeiler (2004) describe subtypes and domains as "advantageous for display performance, geoprocessing and management” and "data integrity," respectively. Table 5-2 contains a list of additional advantages presented in the Building Geodatabases (ESRI, 2006b). Standardizing data in the geodatabase became crucial for (a) facilitating data entry, (b) ensuring data quality, and (c) building a solid foundation for the web-GIS application.

Table 5-2. Advantages of Subtypes and Domains

\begin{tabular}{|l|l|}
\hline \multicolumn{1}{|c|}{ Subtypes } & \multicolumn{1}{c|}{ Domains } \\
\hline - Manage groups of features & • Prevent and locate attribute error \\
- List valid values & • Maintain consistent coding schemes \\
- Locate attribute errors & - Facilitate data entry \\
- Apply default values & - Copied during data conversion \\
- Locate spatial errors & - Reduce database size \\
- More efficient editing & \\
\hline
\end{tabular}

The purpose of creating subtypes and domains was to facilitate quick and consistent data entry by providing data editors with a list of valid values for each ecotourism service. Data entry is quicker when choosing an attribute value from a drop-down list than typing the same information in the attribute field. Facilitating quick data entry was intended to encourage future data collection, editing, and maintenance of the data. In addition, it was important to ensure data consistency in the geodatabase, since the web-GIS application must conduct a systematic query on specific values in specific fields in order to successfully retrieve data and display it online.

Only one subtype per feature class can be created but multiple domains can be created per feature class. The TourismServices_PA feature class has one subtype and multiple domains. Figure 5-3 illustrates the subtype applied to the ecotourism service point feature class. The TourismServices_PA feature class (see $\mathbf{0}$ ) was categorized into 13 
subtypes (see 2). In other words, every ecotourism services point data falls under one of the 13 pre-defined categories. Coded values, or domains, were then created for each ecotourism service subtypes (see 3).

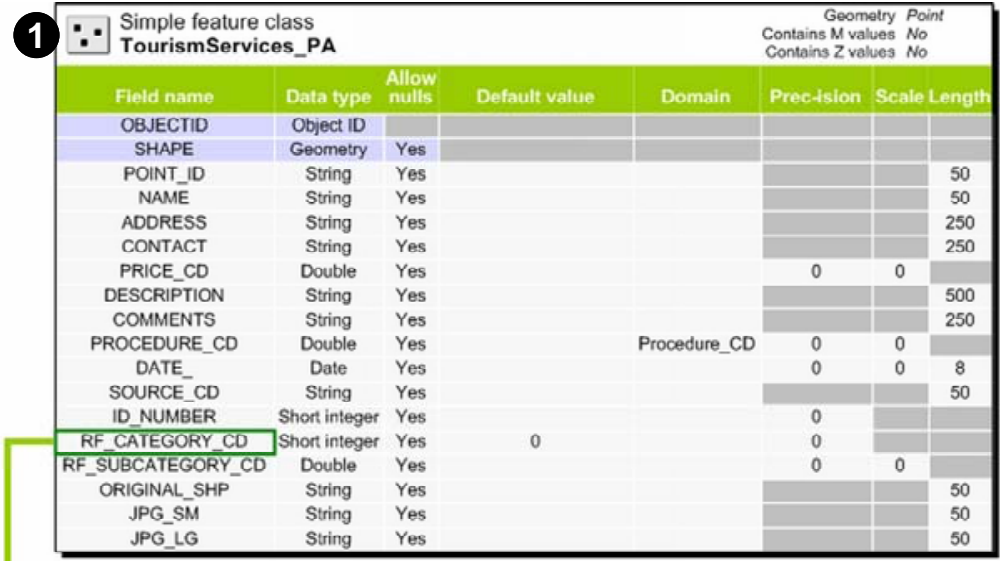

\begin{tabular}{|c|c|c|c|}
\hline $\begin{array}{l}\text { Subtyp } \\
\text { Sub } \\
\text { Defaut }\end{array}$ & $\begin{array}{l}\text { pes of TourismServices_PA } \\
\text { lbtype field RF_CATEGORY_CD } \\
\text { ut subtype } 0\end{array}$ & List of defined dofault v: & $s$ and domains for subtypes in this class \\
\hline $\begin{array}{l}\text { Sublype } \\
\text { Code }\end{array}$ & $\begin{array}{l}\text { Subtype } \\
\text { Description }\end{array}$ & Field name & Default value \\
\hline 1 & Accommodation & $\begin{array}{c}\text { PRICE_CD } \\
\text { PROCEDURE_CD } \\
\text { SOURCE_CD } \\
\text { RF_SUBCATEGORY_CD }\end{array}$ & $\begin{array}{c}\text { AccommodationPrice_CN } \\
\text { Procedure_CD } \\
\text { Source_CD } \\
\text { Accommodation_CD }\end{array}$ \\
\hline 2 & Agencies & $\begin{array}{c}\text { PRICE_CD } \\
\text { PROCEDURE_CD } \\
\text { SOURCE_CD } \\
\text { RF_SUBCATEGORY_CD }\end{array}$ & $\begin{array}{l}\text { OtherPrice_CN } \\
\text { Procedure_CD } \\
\text { Source_CD } \\
\text { Agencies_CD }\end{array}$ \\
\hline 3 & Communication & $\begin{array}{c}\text { PRICE_CD } \\
\text { PROCEDURE_CD } \\
\text { SOURCE_CD } \\
\text { RF_SUBCATEGORY_CD }\end{array}$ & $\begin{array}{l}\text { OtherPrice_CN } \\
\text { Procedure_CD } \\
\text { Source_CD } \\
\text { Communications_CD }\end{array}$ \\
\hline 4 & Education & $\begin{array}{c}\text { PRICE_CD } \\
\text { PROCEDURE_CD } \\
\text { SOURCE_CD } \\
\text { RF_SUBCATEGORY_CD }\end{array}$ & $\begin{array}{l}\text { OtherPrice_CN } \\
\text { Procedure_CD } \\
\text { Source_CD } \\
\text { Education_CD }\end{array}$ \\
\hline 5 & Financial & $\begin{array}{l}\text { PRICE_CD } \\
\text { PROCEDURE_CD } \\
\text { SOURCE_CD } \\
\text { RF_SUBCATEGORY_CD }\end{array}$ & $\begin{array}{l}\text { OtherPrice_CN } \\
\text { Procedure_CD } \\
\text { Source_CD } \\
\text { Financial_CD }\end{array}$ \\
\hline 6 & Food/Entertainment $\Rightarrow$ & $\begin{array}{c}\text { PRICE_CD } \\
\text { PROCEDURE_CD } \\
\text { SOURCE_CD } \\
\text { RF_SUBCATEGORY_CD }\end{array}$ & $\begin{array}{c}\text { DiningPrice_CN } \\
\text { Procedure_CD } \\
\text { Source_CD } \\
\text { FoodEntertainment_CD }\end{array}$ \\
\hline 7 & Information Centers & $\begin{array}{l}\text { PRICE_CD } \\
\text { PROCEDURE_CD } \\
\text { SOURCE_CD } \\
\text { RF SUBCATEGORY CD }\end{array}$ & $\begin{array}{l}\text { OtherPrice_CN } \\
\text { Procedure_CD } \\
\text { Source_CD } \\
\text { InfoCenters_CD }\end{array}$ \\
\hline 8 & Medical & $\begin{array}{l}\text { PRICE_CD } \\
\text { PROCEDURE_CD } \\
\text { SOURCE_CD } \\
\text { RF_SUBCATEGORY_CD }\end{array}$ & $\begin{array}{l}\text { OtherPrice_CN } \\
\text { Procedure_CD } \\
\text { Source_CD } \\
\text { Medical_CD }\end{array}$ \\
\hline 9 & Museum/Visitor Centers $\Rightarrow$ & $\begin{array}{l}\text { PRICE_CD } \\
\text { PROCEDURE_CD } \\
\text { SOURCE_CD } \\
\text { RF SUBCATEGORY CD }\end{array}$ & $\begin{array}{c}\text { OtherPrice_CN } \\
\text { Procedure_CD } \\
\text { Source_CD } \\
\text { MuseumVisitorCenter CD }\end{array}$ \\
\hline 10 & Recreation & $\begin{array}{c}\text { PRICE_CD } \\
\text { PROCEDURE_CD } \\
\text { SOURCE_CD } \\
\text { RF_SUBCATEGORY_CD }\end{array}$ & $\begin{array}{c}\text { OtherPrice_CN } \\
\text { Procedure_CD } \\
\text { Source_CD } \\
\text { Recreation_CD }\end{array}$ \\
\hline 11 & Shopping & $\begin{array}{l}\text { PRICE_CD } \\
\text { PROCEDURE_CD } \\
\text { SOURCE_CD } \\
\text { RF SUBCATEGORY CD }\end{array}$ & $\begin{array}{l}\text { OtherPrice_CN } \\
\text { Procedure_CD } \\
\text { Source_CD } \\
\text { Shopping CD }\end{array}$ \\
\hline 12 & Tour Operators & $\begin{array}{c}\text { PRICE_CD } \\
\text { PROCEDURE_CD } \\
\text { SOURCE_CD } \\
\text { RF_SUBCATEGORY_CD }\end{array}$ & $\begin{array}{l}\text { OtherPrice_CN } \\
\text { Procedure_CD } \\
\text { Source_CD } \\
\text { Tour Operators_CD }\end{array}$ \\
\hline 13 & Transportation & $\begin{array}{c}\text { PRICE_CD } \\
\text { PROCEDURE_CD } \\
\text { SOURCE_CD } \\
\text { RF_SUBCATEGORY_CD }\end{array}$ & $\begin{array}{l}\text { OtherPrice_CN } \\
\text { Procedure_CD } \\
\text { Source_CD } \\
\text { Transportation_CD }\end{array}$ \\
\hline
\end{tabular}

Figure 5-3. Ecotourism Services Feature Class and Subtype. 
Subtypes and domains applied to the RF_SUBCATEGORY_CD field were specified by the client (see (1). Subtypes were manually entered into the TourismServices_PA feature class in ArcCatalog. Domains were defined by the client, entered into an Excel spreadsheet (refer to EcotourismServicePA_DOMAIN.xls enclosed on the project CD), and exported into the "SustainableEcotourism" FGDB using the "Table to Domain" tool in ArcToolbox. Domains may be updated and re-exported into the geodatabase to replace the old one at anytime using the same tool.

The TourismService_PA feature class table has a field that records the data collection method of each point. All points in this feature class were either (a) collected by handheld GPS units, (b) digitized, (c) interpreted from the Internet source, (d) book source, (e) existing map, or (f) arbitrarily created for development purposes. This information was applied as a domain to further facilitate data entry (see (2).

Another set of domain is applied to the PRICE_CD field of the TourismService_PA feature class (see 3). This information was extracted from books and Internet sources, as previously discussed in Chapter 4. Once again, this information is useful for tourists in determining where to stay overnight or dine while they visit the protected areas.

Figure 5-4 shows examples of coded values associated with the Accommodation subtype for the attribute field, RF_SUBCATEGORY_CD.
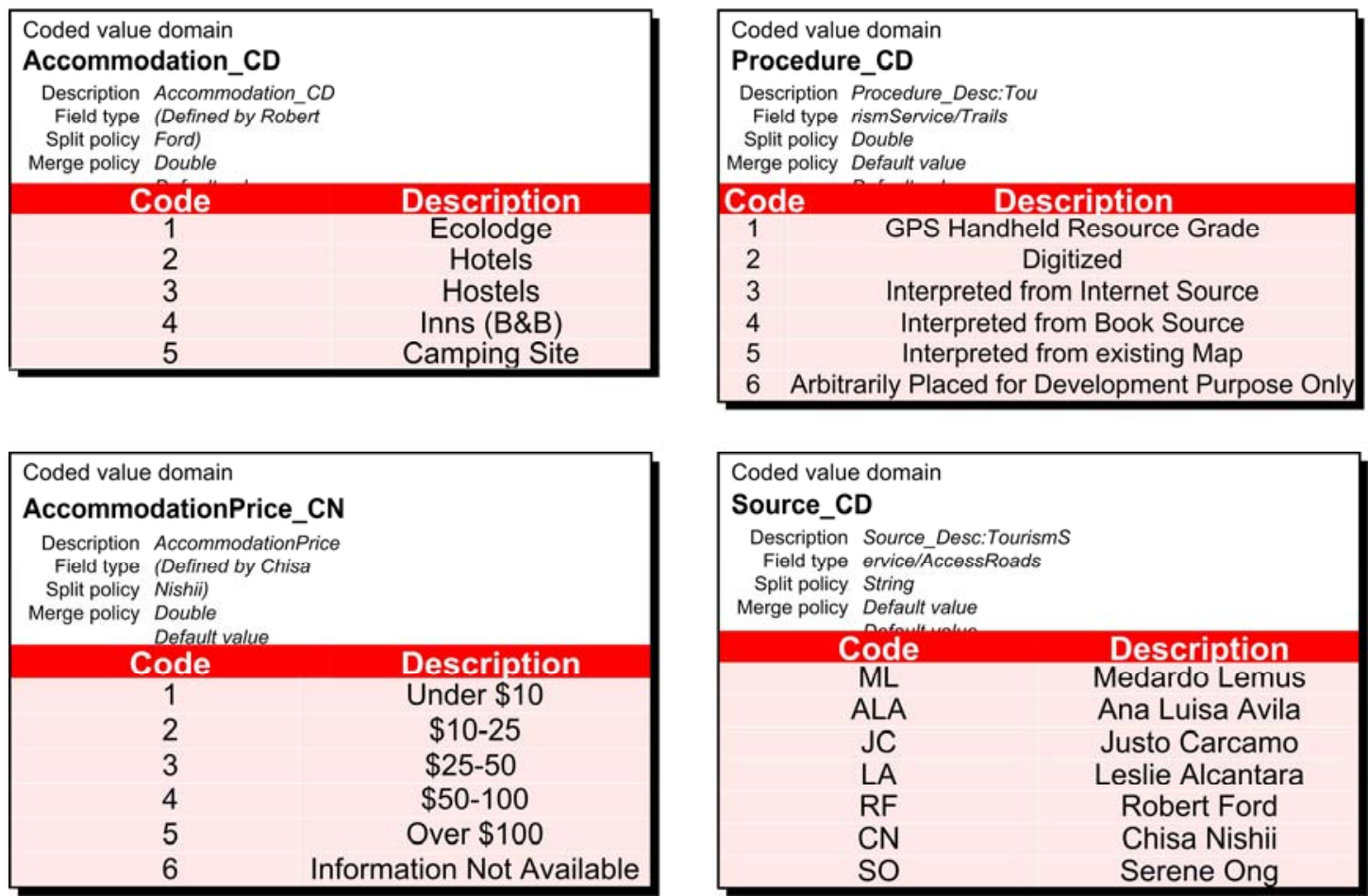

\begin{tabular}{|cc|}
\hline \begin{tabular}{l} 
Coded value domain \\
Source_CD \\
Description Source_Desc:Tourisms \\
Field type ervice/AccessRoads \\
Split policy String \\
Merge policy Default value \\
\hline Code
\end{tabular} \\
\hline ML & Description \\
\hline ALA & Medardo Lemus \\
JC & Ana Luisa Avila \\
LA & Justo Carcamo \\
RF & Leslie Alcantara \\
CN & Robert Ford \\
SO & Chisa Nishii \\
\hline
\end{tabular}

Figure 5-4. Domains for Accommodation Subtypes. 
Once subtypes and domains are applied, drop-down lists appear while editing the TourismService_PA feature class in ArcMap. Figure 5-5 is a comparison of drop-down lists populated with domains associated with two different subtypes: accommodation and food/entertainment.

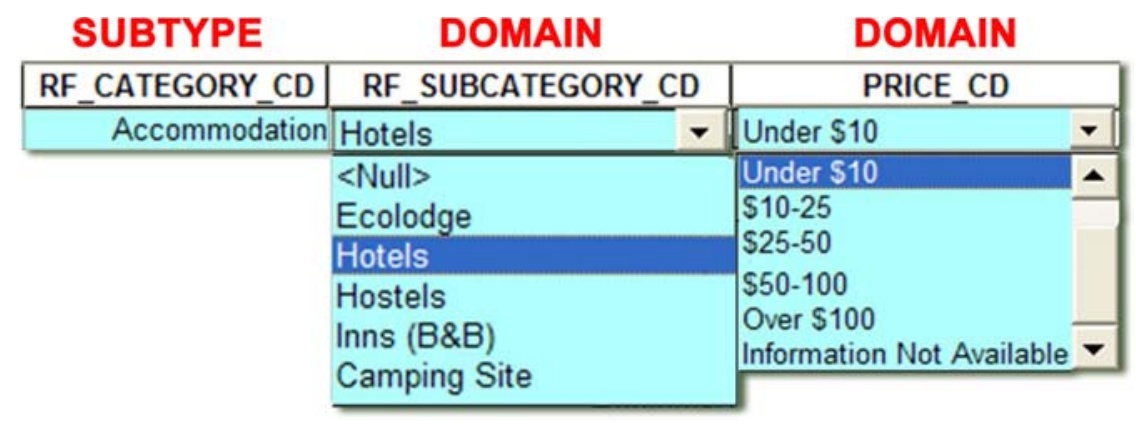

\begin{tabular}{|c|c|c|c|}
\hline RF_CATEGORY_CD & \multicolumn{2}{|c|}{ RF_SUBCATEGORY_CD } & PRICE_CD \\
\hline \multicolumn{2}{|c|}{ Food/Entertainment } & & Mid Range \\
\hline & $<$ Null $>$ & 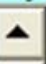 & $\begin{array}{l}\text { Budget } \\
\text { Mid Ranoe }\end{array}$ \\
\hline & $\begin{array}{l}\text { Bar } \\
\text { Bakery }\end{array}$ & & $\begin{array}{l}\text { Top End } \\
\text { Information Not Available }\end{array}$ \\
\hline & $\begin{array}{l}\text { Cafeteria } \\
\text { Market } \\
\text { Restaurant } \\
\text { Local Eateries } \\
\text { Food Court } \\
\text { Fast Food } \\
\text { Liquor Store } \\
\text { Club }\end{array}$ & $\nabla$ & \\
\hline
\end{tabular}

Figure 5-5. Example of Subtypes and Domains in ArcMap Editor. 



\section{WebGIS Development}

This chapter discusses the details entailed in building and customizing a web-GIS application. Two websites were created for this project: (a) one basic website with no customization upon a very specific request from the client for a webpage with low maintenance requirements, and (b) another website with customization to explore the benefits offered to the user.

\subsection{ArcGIS Server Default Website}

The first website was created as a design prototype to assess the client's needs. Below are the basic procedures that were followed to create the website.

1. Created a base map document.

2. Connected to GIS Server.

3. Published the map document in ArcCatalog.

4. Created a new webpage using the Web Mapping Application template.

5. Added the map service to the MapResourceManager using ArcGIS Server Local.

6. Added ArcGIS Identity in the solution explorer window.

\subsubsection{Map Design}

The first website was created in several phases, with the initial phase being the creation of the base map document (Figure 6-1). This initial phase was critical in determining the appearance of the final website that was published online.

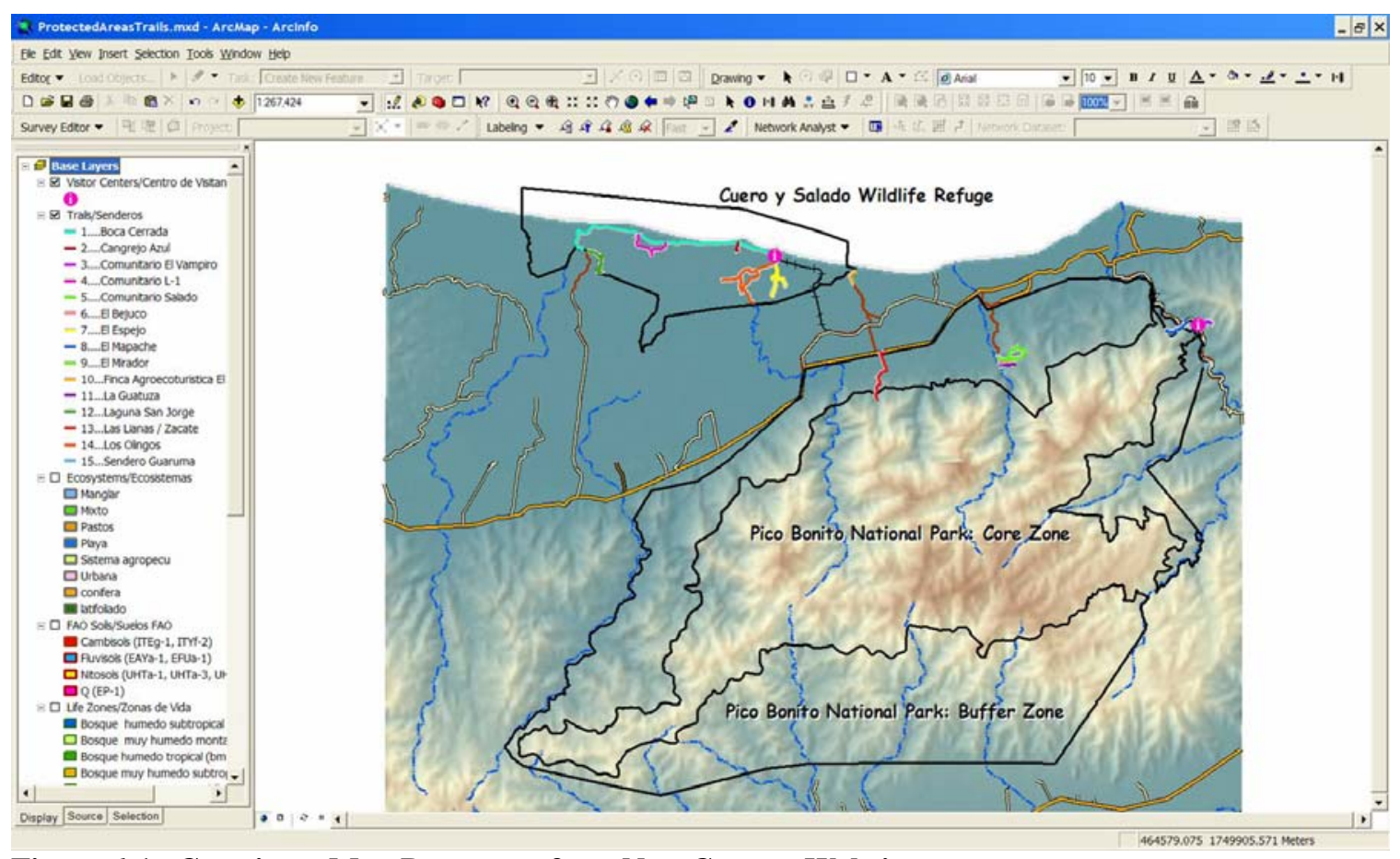

Figure 6-1. Creating a Map Document for a Non-Custom Website. 
The client defined the crucial data layers to be displayed for this website. Data layers specified included roads, rivers, trails, two visitor centers (TourismService_PA feature class), ecosystems, life zones, forestry, scanned topo image, satellite image, and hillshade. After the data layers were specified, cartographic techniques were employed to enhance the map display interface. Among other map design elements that were considered (e.g., font type, color, size), particular attention was given to two map design elements: symbology and scale dependency.

Symbology of each data layer was carefully selected to optimize visibility of each data layer and corresponding labels across various scales and image backgrounds. For example, bright colors were selected to symbolize trails so that each of the 15 trails could be easily distinguished from one another across varied scales. The cased-line symbols, two-line segments of varied width on top of each other, were used to display trails. This allowed all the trails to have thicker gray shades behind to give them a two-dimensional look and raise the feature within the visual hierarchy.

Building scale dependencies on both data layers and labels ensured (a) avoiding clutter and less informative display, and (b) improved response time. In some cases, the scale dependencies of the labels were set to larger scale than the corresponding data layers to avoid clutter. The processing and download time are directly linked to the number of features being displayed. Hence, the less-detailed national roads and rivers were displayed only at a small scale, whereas detailed regional roads and rivers were displayed only on large scale map displays. Table 6-1 summarizes the scale dependency of the data layers and corresponding labels built in the map document.

Table 6-1. Layer and Label Scale Thresholds

\begin{tabular}{|c|c|c|c|c|}
\hline & \multicolumn{2}{|c|}{ Layer } & \multicolumn{2}{|c|}{ Label } \\
\hline Feature Class Name & $\begin{array}{c}\text { Minimum } \\
\text { Scale }\end{array}$ & $\begin{array}{l}\text { Maximum } \\
\text { Scale }\end{array}$ & $\begin{array}{l}\text { Minimum } \\
\text { Scale }\end{array}$ & $\begin{array}{l}\text { Maximum } \\
\text { Scale }\end{array}$ \\
\hline ProtectedAreas MIP & \multicolumn{2}{|c|}{ Show Always } & None & $1: 200,000$ \\
\hline RoadsSS MIP & None & $1: 80,000$ & $1: 150,000$ & None \\
\hline RoadsLS_MIP & $1: 80,000$ & None & \multicolumn{2}{|c|}{ No Label } \\
\hline RiversSS_MIP & None & $1: 80,000$ & $1: 150,000$ & $1: 50,000$ \\
\hline RiversLS_MIP & $1: 80,000$ & None & \multicolumn{2}{|c|}{ No Label } \\
\hline Forestal95_MIP & \multicolumn{2}{|c|}{ Show Always } & \multicolumn{2}{|c|}{ No Label } \\
\hline Ecosystems_MIP & \multicolumn{2}{|c|}{ Show Always } & \multicolumn{2}{|c|}{ Show Always } \\
\hline SoilsFAO_MIP & \multicolumn{2}{|c|}{ Show Always } & \multicolumn{2}{|c|}{ Show Always } \\
\hline ZonasVida_MIP & \multicolumn{2}{|c|}{ Show Always } & \multicolumn{2}{|c|}{ Show Always } \\
\hline AccessRoads & \multicolumn{2}{|c|}{ Show Always } & $1: 150,000$ & None \\
\hline Trails & \multicolumn{2}{|c|}{ Show Always } & $1: 130,000$ & None \\
\hline EcotourismServices PA & \multicolumn{2}{|c|}{ Show Always } & None & None \\
\hline PointsInterest & \multicolumn{2}{|c|}{ Show Always } & \multicolumn{2}{|c|}{ No Label } \\
\hline Raster Dataset Name & $\begin{array}{l}\text { Minimum } \\
\text { Scale }\end{array}$ & $\begin{array}{l}\text { Maximum } \\
\text { Scale }\end{array}$ & $\begin{array}{l}\text { Minimum } \\
\text { Scale }\end{array}$ & $\begin{array}{l}\text { Maximum } \\
\text { Scale }\end{array}$ \\
\hline HondurasTopo & $1: 130,000$ & None & \multicolumn{2}{|c|}{ No Label } \\
\hline HondurasLANDSAT & $1: 200,000$ & None & \multicolumn{2}{|c|}{ No Label } \\
\hline HondurasDEM & None & $1: 200,000$ & \multicolumn{2}{|c|}{ No Label } \\
\hline HondurasRCH & None & $1: 200,000$ & \multicolumn{2}{|c|}{ No Label } \\
\hline HondurasMFH & None & $1: 200,000$ & \multicolumn{2}{|c|}{ No Label } \\
\hline HondurasMDOW & None & $1: 200,000$ & \multicolumn{2}{|c|}{ No Label } \\
\hline
\end{tabular}


As listed in Table 6-2, there are five major scale breaks, or thresholds. These were categorized in three main scale classes for simplicity: small, medium, and large.

Table 6-2. Scale Categories

\begin{tabular}{|c|c|}
\hline Scale Category & Scale \\
\hline Small Scale & $1: 200,000$ and Up \\
\hline & $1: 150,000$ \\
\hline Medium Scale & $1: 130,000$ \\
\hline & $1: 80,000$ \\
\hline Large Scale & $1: 50,000$ and Below \\
\hline
\end{tabular}

\section{$\underline{\text { Hyperlinks }}$}

External links were added to the webpage by configuring the web.sitemap file (Refer to Appendix B for code). Below are additional functionalities added to the webpage. Hyperlinks were created to retrieve relevant information listed below:

- Pico Bonito National Park homepage

- Cuero y Salado Wildlife Refuge homepage

- University of Redlands MS GIS Program webpage

- A Microsoft power point show describing the project overview (Appendix C)

- A Microsoft power point show describing how to use this webpage (Appendix D)

- MailTo functionality to allow users to email directly to the author for comments and suggestions regarding the project or website.

\subsection{Customization}

The second website focused on customization. The basic procedure in creating a website was the same as the procedures in creating the first website (Refer to Section 6.1). What make the second website unique were (a) custom web mapping application template, (b) the custom table of contents, (c) select and zoom-in functionality, (d) Image hyperlink and description, (e) multiple-select functionality, and (f) MapTips. Each of these customizations is discussed in detail below.

A custom web mapping application was built using the Visual Studio 2005 .NET development environment (Figure 6-2). This figure will be referred to frequently in order to visually indicate where customization occurred. 


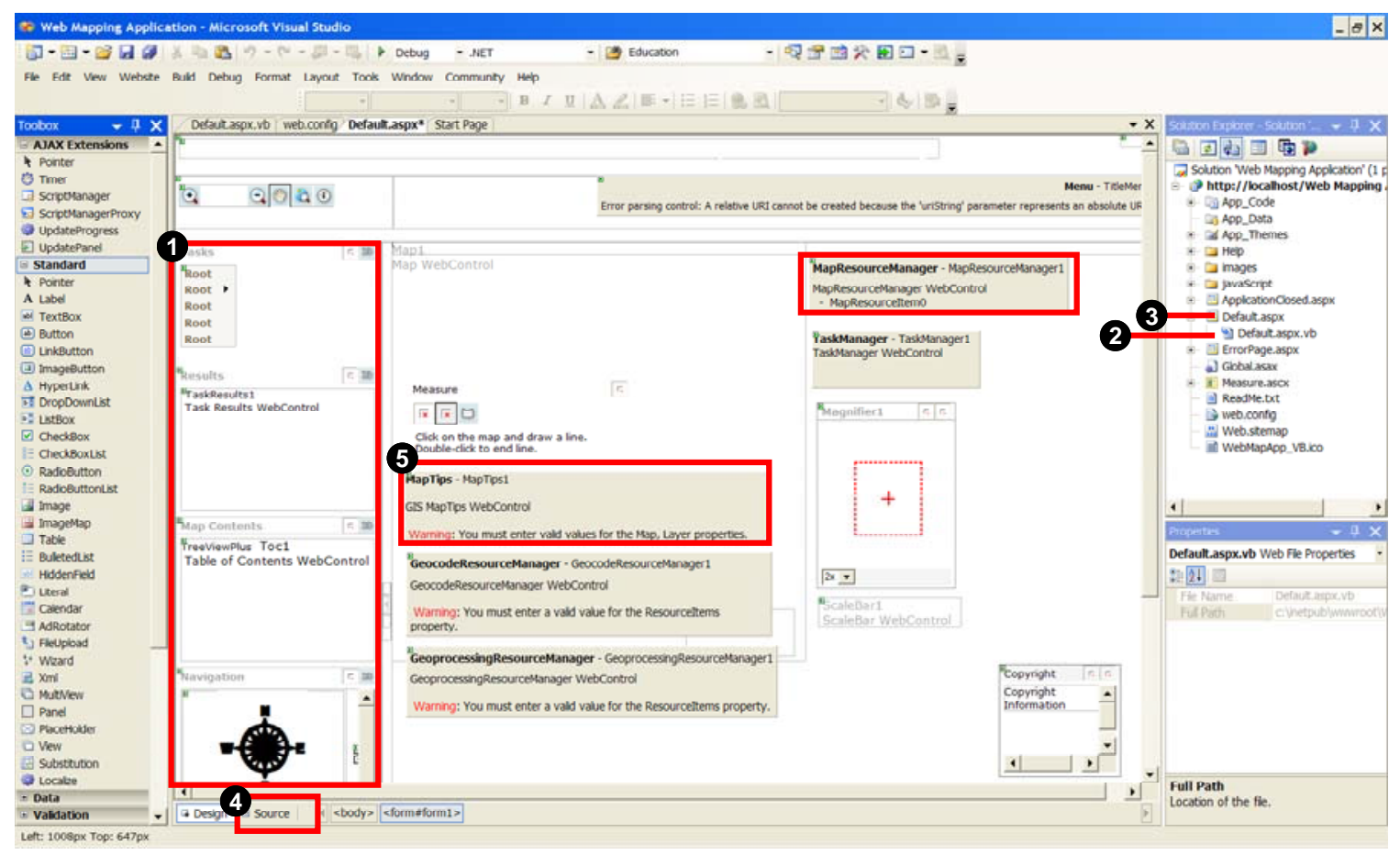

Figure 6-2. Visual Studio 2005 .NET Development Environment.

\subsubsection{Custom Mapping Application Template}

The web mapping application template was slightly modified for the ecotourism services website, which provided a good foundation to build a custom application. This process involved combining two website templates: the ESRI Web Mapping Application template and the AJAX Enabled Website template.

The web mapping application template comes with the Web Application Developer Framework (ADF). ArcGIS ADF contains specialized ESRI controls embedded into the Visual Studio .NET, and these were utilized to add various web mapping functionalities to the ecotourism service website. In addition, ASP.NET AJAX web controls were incorporated in order to refresh selected components of the webpage independently, instead of refreshing the entire page. Figure 6-3 shows all web ADFs used to customize the webpage for this project. 


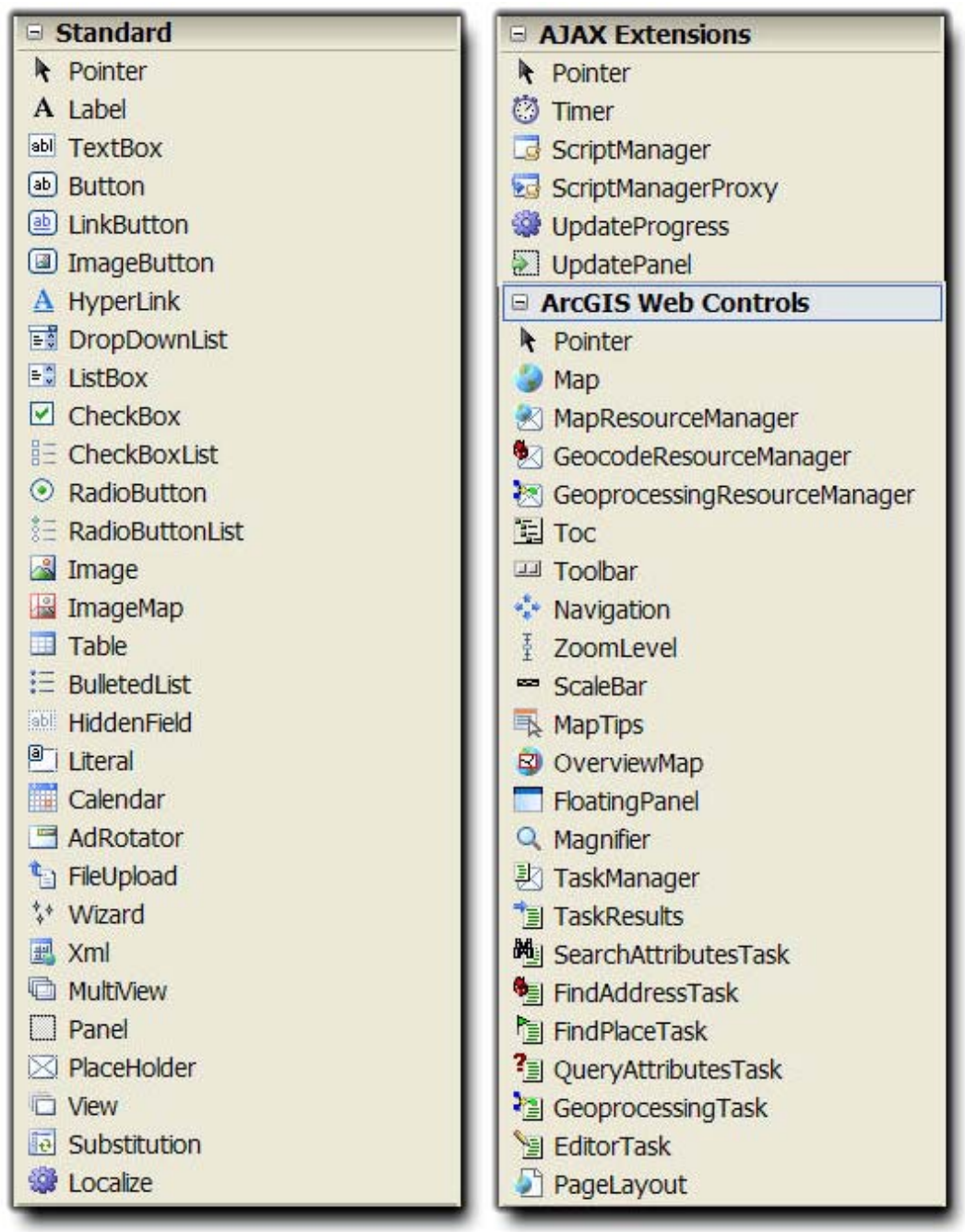

Figure 6-3. List of Web Controls Used for the Customization.

Creating a new Web Mapping Application Template involved two steps:

1. The Microsoft ASP .NET AJAX extension for Visual Studio 2005 was downloaded $^{14}$ and installed.

2. The AJAX Enabled Website template and the ESRI Web Mapping Application template were combined by copying the web.config entries from the AJAX Enabled Webpage to the ESRI Web Mapping Application web.config.

The resulting AJAX-enabled custom application template configuration code is shown in Appendix E.

\subsubsection{Custom Table of Contents}

A custom table of contents was created to increase the visibility of the symbols and to provide the table with a cleaner look. As illustrated in Figure 6-4, symbols are difficult to see in the default table of contents. Symbols are collapsed into the theme layers and are

\footnotetext{
${ }^{14}$ Microsoft ASP.NET AJAX Site: http://ajax.asp.net
} 
accessible only when users expand the layers. The custom table of contents, on the other hand, can display the symbol next to the layer name, significantly increasing the visibility of the symbols and reducing screen space. More importantly, the web administrator has control over what layers to make available for the Internet audience to turn on and off.
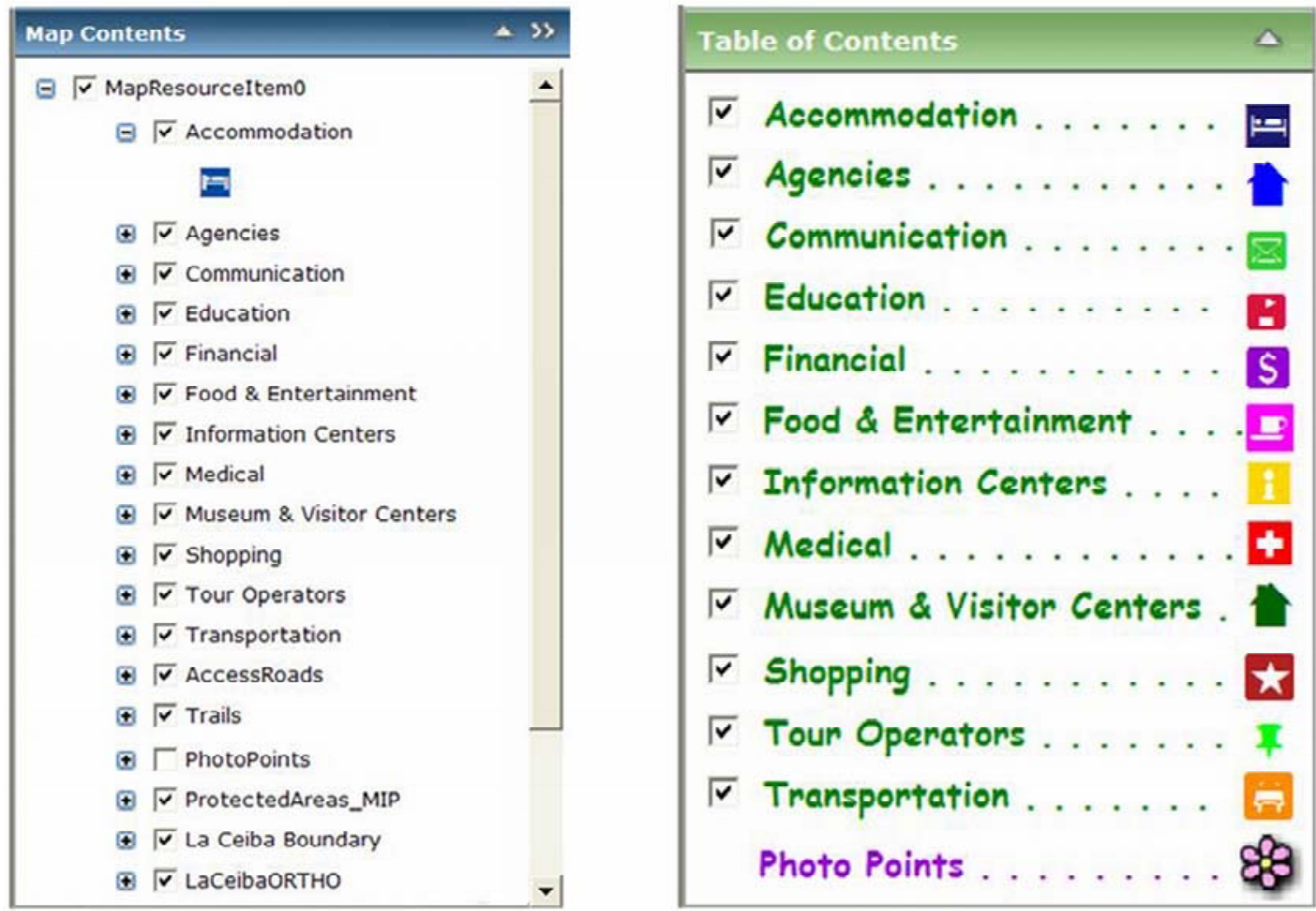

Figure 6-4. Default Table of Contents vs. Custom Table of Contents.

Creating a custom table of contents involved the following steps.

1. Dropped an ArcGIS FloatingPannel to the left panel (Figure 6-2 $\mathbf{0}$ ) and named it Table of Contents.

2. Dropped an AJAX UpdatePanel in the new Table of Contents panel.

3. Dropped 12 Standard CheckBoxes in the AJAX UpdatePanel and named each of them (e.g., Accommodation, Agencies, Communication)

4. Enable Autopostback of the CheckBoxes.

5. Added code (APPENDIX F) to the Default.aspx.vb (Figure 6-2 2) to turn ON and OFF the individual layers when checked boxes are turned ON and OFF, respectively.

6. Dropped a Standard Image control in the Table of Contents panel and referenced it to an image showing all 13 ecotourism service symbols.

\subsubsection{Select and Zoom-In Functionality}

For quick and easy access to data, a custom legend was created with ecotourism services features grouped by subtypes onto tabs. Similarly, two pull-down lists were built for each tab to provide the users with a list of pre-defined values from which to choose. 
Once values were selected from the drop-down lists, the map would then zoom to the specific ecotourism service location. For simplicity, the first drop-down list will be referred as the category drop-down list and the second as the subcategory drop-down list.

Below are steps undertaken to add the select and automatic zoom-in functionality into the ecotourism services website. These are procedures for adding the accommodation type only. The same procedures were repeated to create the tabs with other ecotourism service categories.

1. Dropped an ArcGIS FloatingPannel in the left panel and named it Accommodation.

2. Dropped an AJAX UpdatePanel in the new Accommodation panel.

3. Dropped a drop-down list in the AJAX UpdatePanel.

4. Enabled Autopostback of the drop-down list and added Accommodation types in the Default.aspx (Figure 6-2 3) source code (Figure 6-2 4).

5. Dropped a subcategory drop-down list in the AJAX UpdatePanel. Enabled Autopostback.

6. Added code (APPENDIX H) to the category drop-down list click event to query the geodatabase and added entries to the subcategory drop-down list based on the item selected.

7. Added code (APPENDIX I) to the second drop-down list click event to change the map extent and performed a callback to refresh the map.

The Visual Basic (VB) code used in this application originated in the ESRI's Developer Network Website $^{15}$. The example code to select features was initially written in C\# but was subsequently translated to VB code and applied to this website. The programming code appears long and rather intimidating, but the logic behind it is straightforward. On the first drop-down list, or the category list, domains were hard-coded. Accommodation, for instance, has five subcategories. The HTML code with those five values added into the category drop-down list looks as follows. See APPENDIX G for complete configuration code.

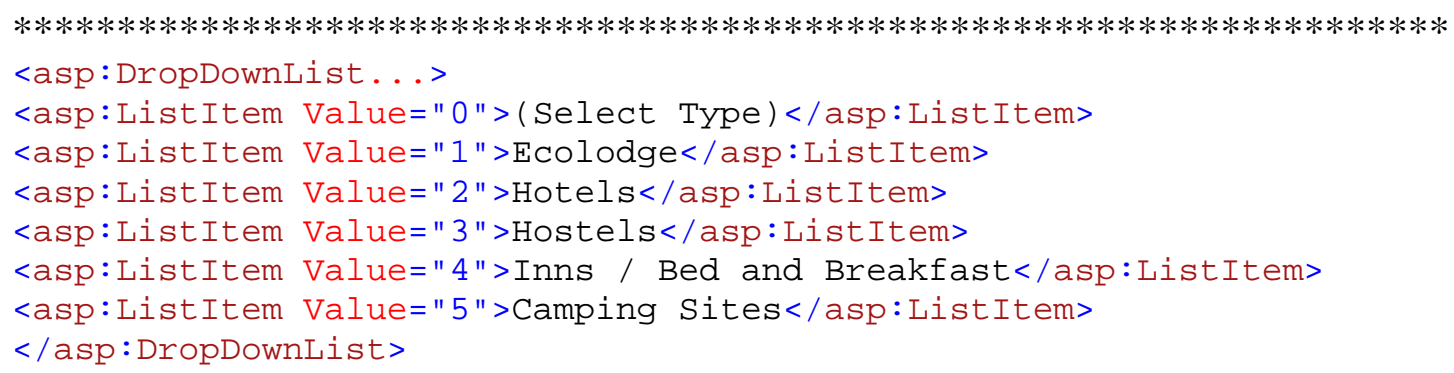

15 ESRI Developer Network Website. Example code was retrieved from: http://edndoc.esri.com/arcobjects/9.2/NET_Server_Doc/developer/ADF/graphics.htm 
Figure 6-5 shows the resulting category drop-down list populated with possible accommodation types.

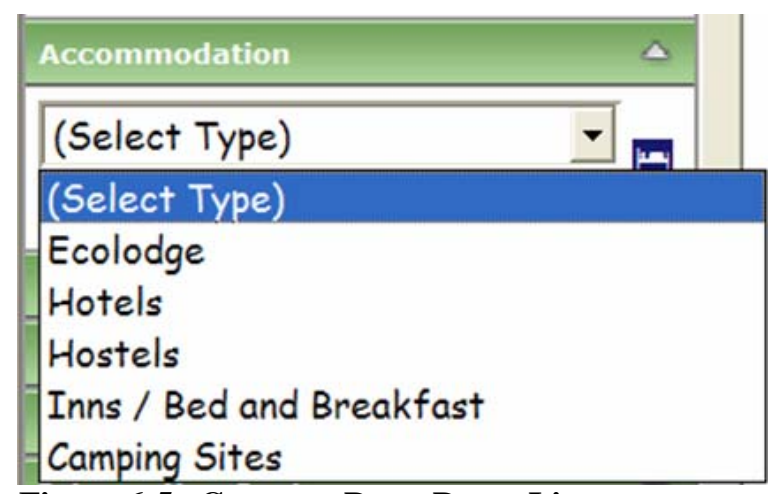

Figure 6-5. Category Drop-Down List

The second drop-down list, or the subcategory list, appears once the user selects a predefined value in the category drop-down list. When a feature is selected from the category drop-down list, the following sequence of events occurs.

First, the Web ADF performs a query on the database. For instance, if Ecolodge is selected, the subcategory drop-down list is populated with names of individual ecolodges. The same thing happens when other accommodation types are selected.

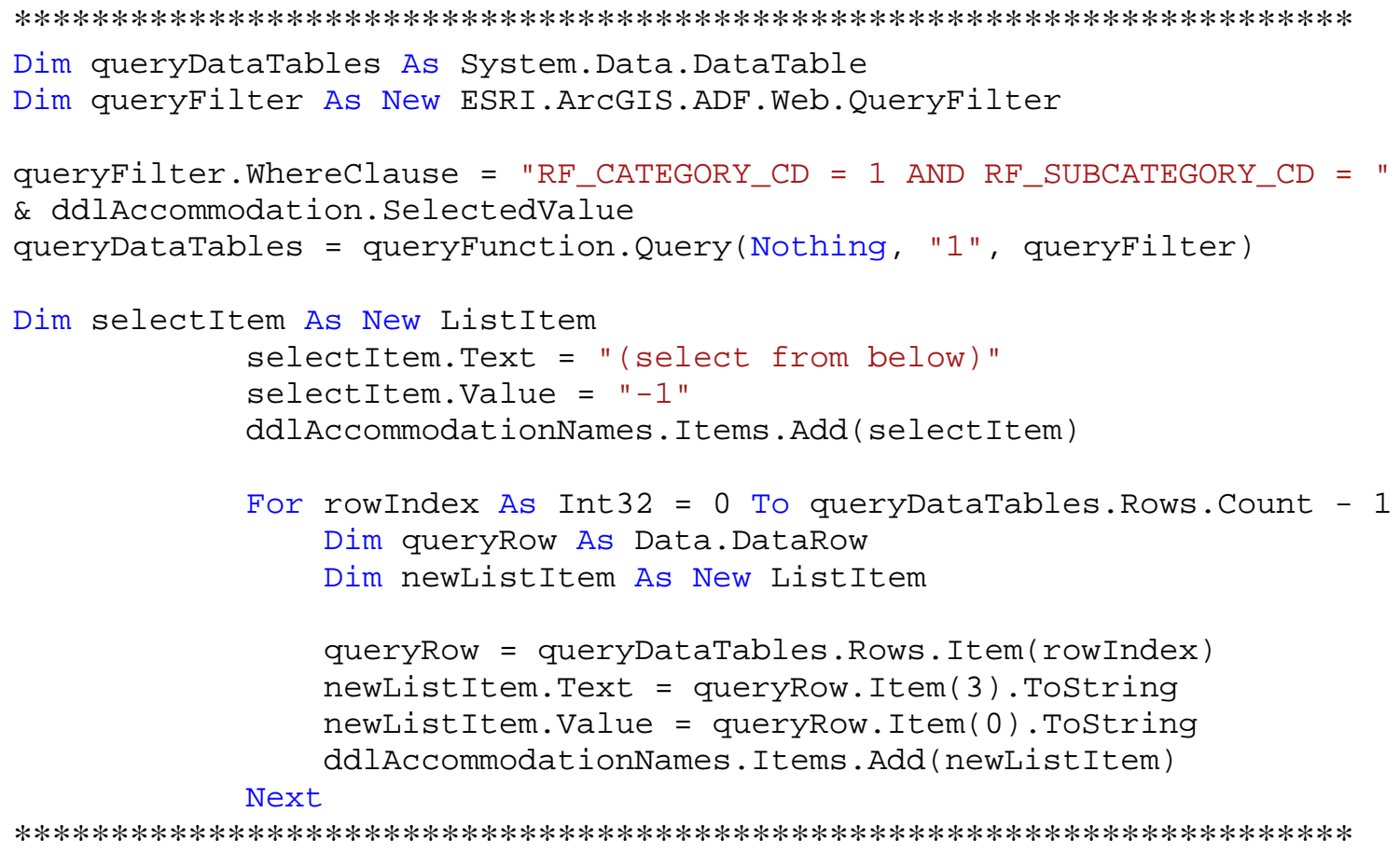

$* * * * * * * * * * * * * * * * * * * * * * * * * * * * * * * * * * * * * * * * * * * * * * * * * * * * * * * * * * * * * * * * * * * * *$ 
Figure 6-6 shows the resulting subcategory drop-down populated with individual ecolodges found in the vicinity of the protected areas.

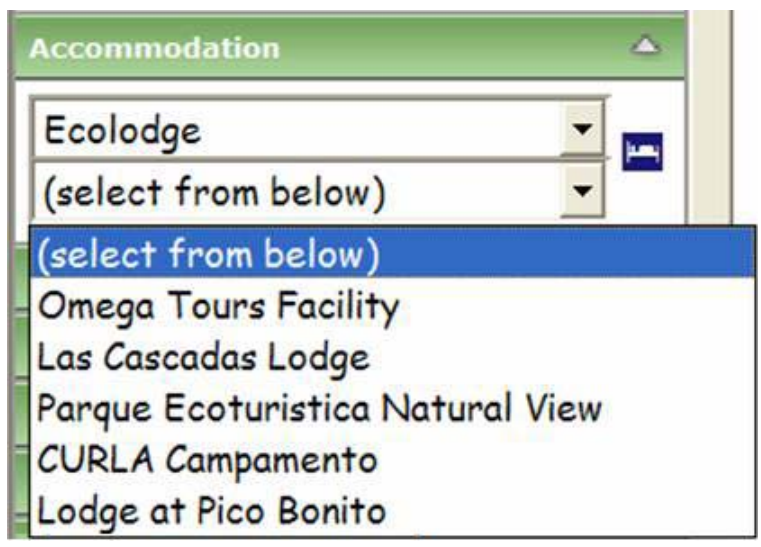

Figure 6-6. Subcategory Drop-Down List.

Secondly, when a feature is selected from the subcategory drop-down list, the query returns a single feature and zooms to that feature at a specified scale. The zoom factor is hard-coded as “Map1.Scale/12000.0” below. The zoom into the feature functionality is triggered by the Zoom function.

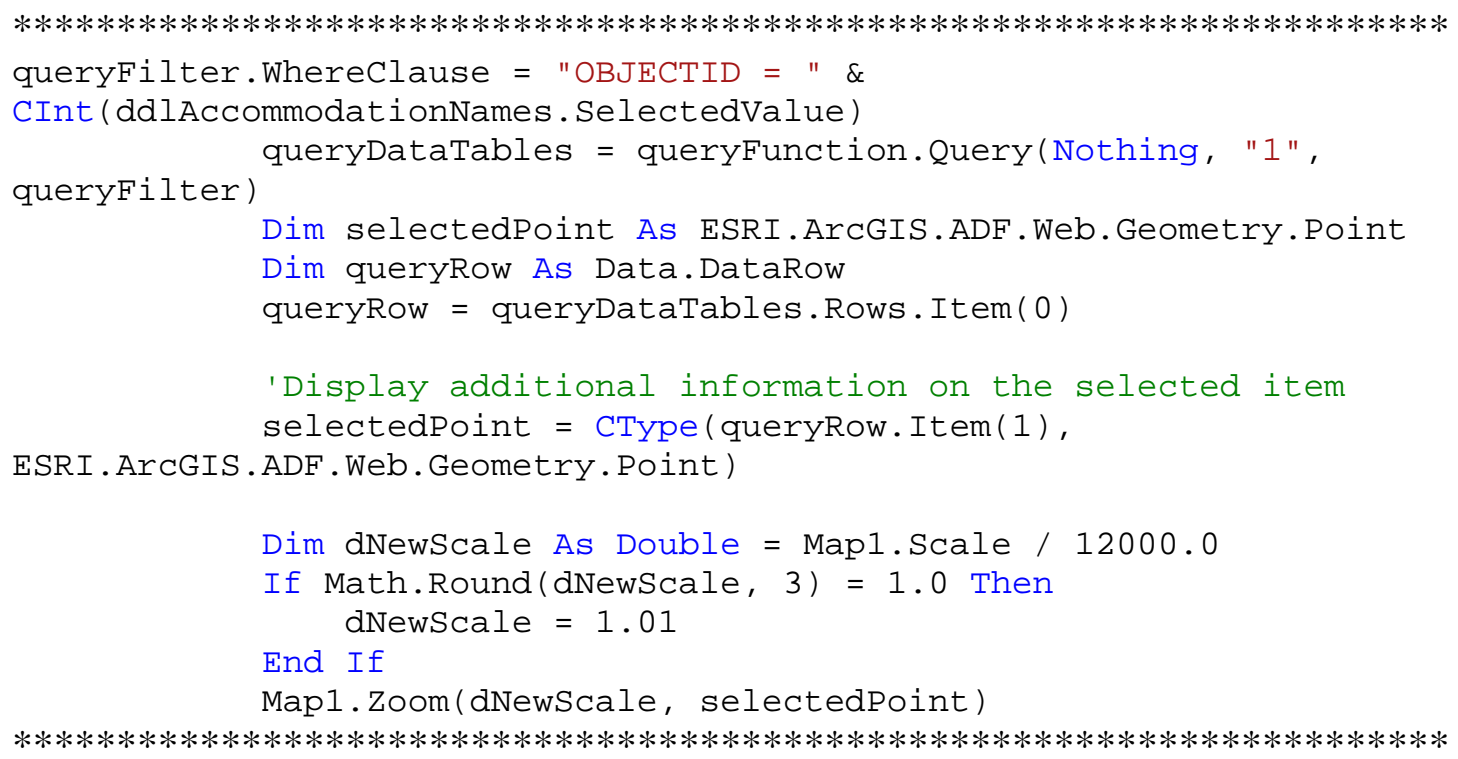


Figure 6-7 illustrates the resulting zoom to feature functionality incorporated in the website as a result of the VB code presented above.

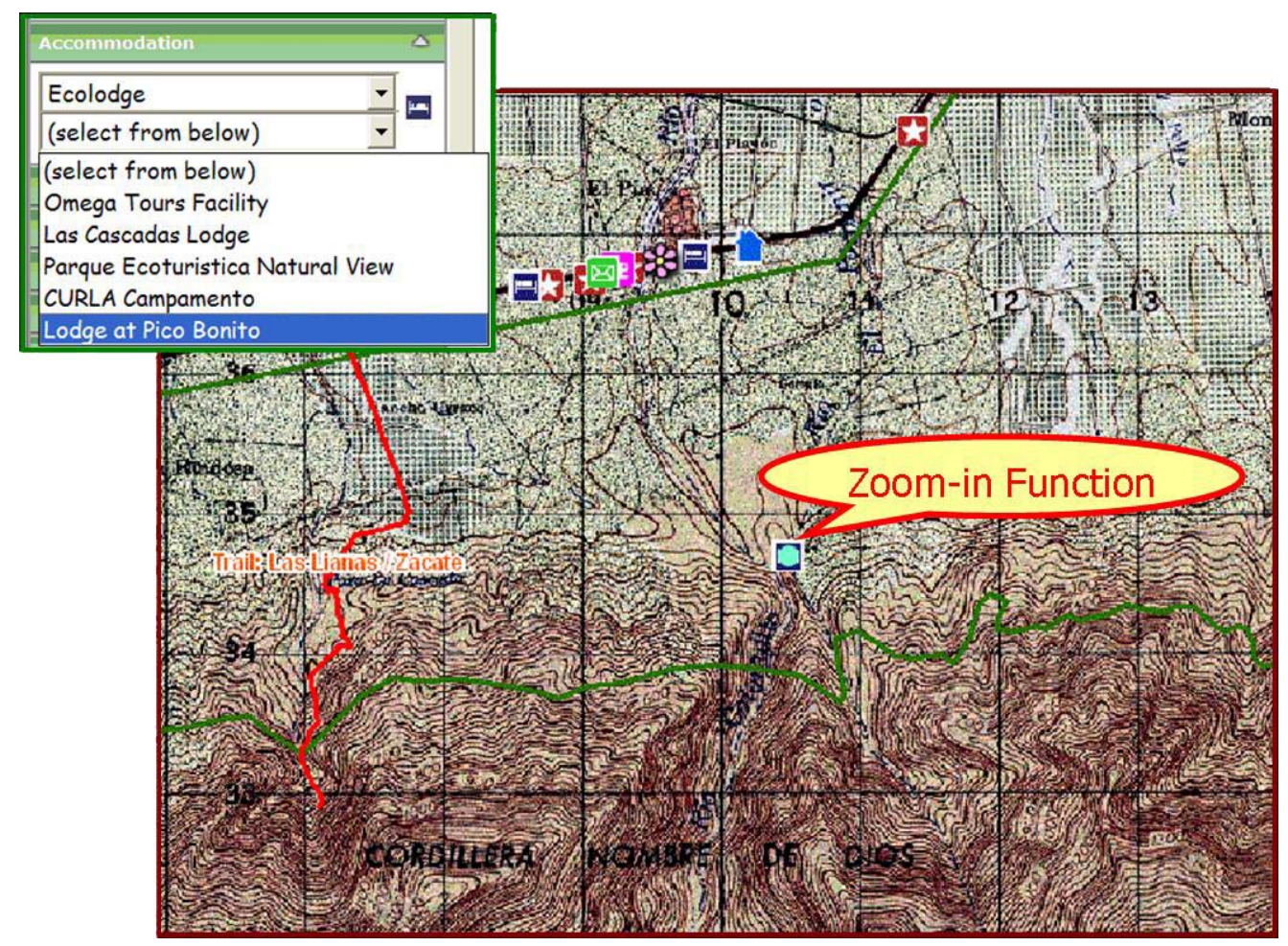

Figure 6-7. Zoom to Feature Functionality.

\subsubsection{Image Hyperlink and Description}

This website was customized to disseminate attribute information for the selected feature. This was accomplished by creating a custom hyperlink query to image names and attribute information stored in the feature class.

Below are four steps undertaken to add the select and automatic zoom-in functionality into the ecotourism services website. Below are procedures of adding the accommodation type only. The same procedures were repeated to create the tabs with other ecotourism service categories.

1. Dropped an ArcGIS FloatingPannel to the left panel and named it Description.

2. Dropped an AJAX UpdatePanel in the new Description panel.

3. Dropped Standard labels in the AJAX UpdatePanel.

4. Added code to the Default.aspx.vb

The website was developed to display photographs associated with each ecotourism point feature. This functionality was added by modifying the Default.aspx.vb to query photos stored in the IMAGE folder.

The VB code that performs this task is shown below. As discussed in the geodatabase design section, the price information for Accommodation is stored as domains and integers. Select case was utilized to convert those integers into price ranges. 


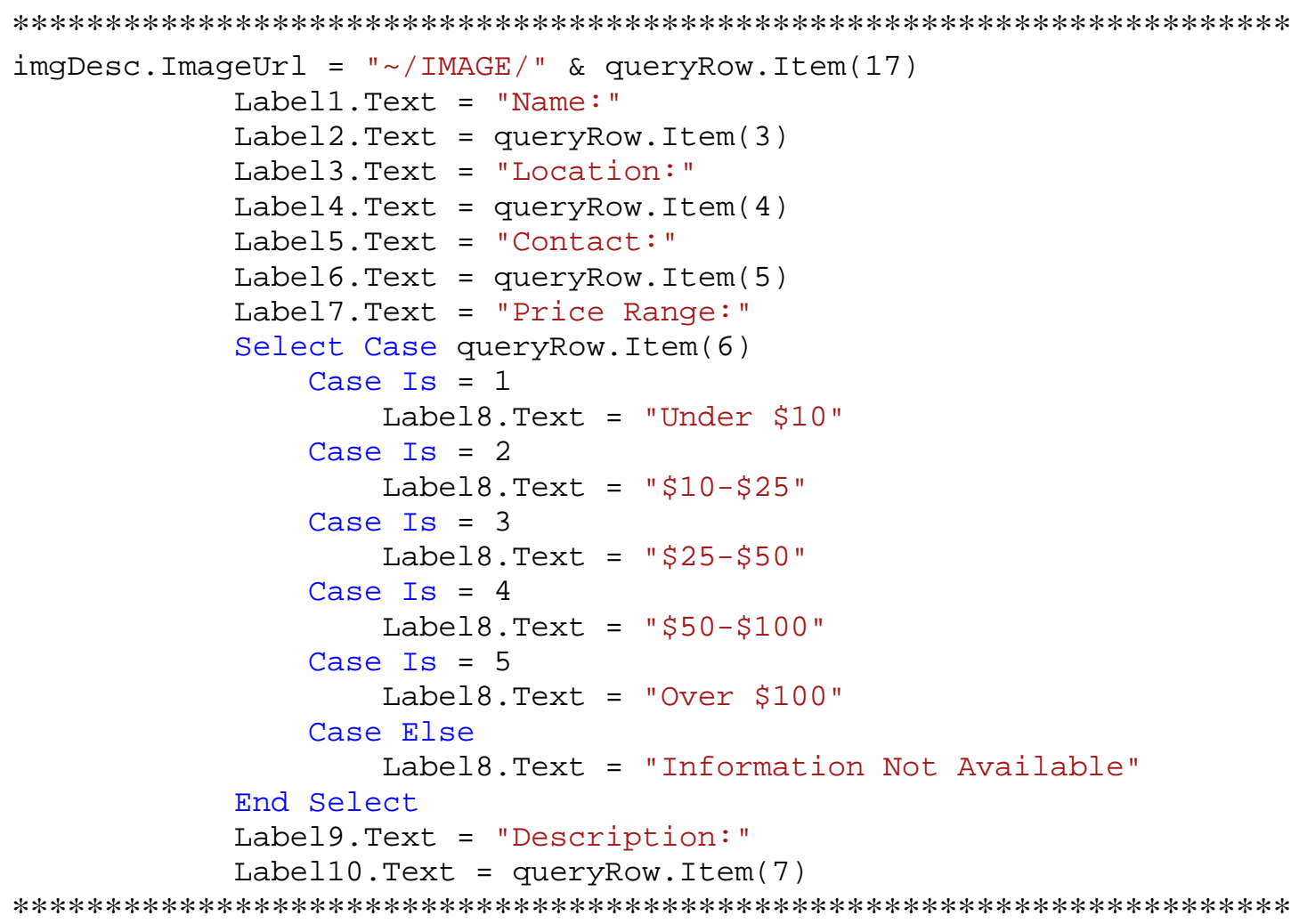

Figure 6-8 shows the resulting graphic interface. The server conducts a database query and displays the attribute information, including the name of the feature, location, contact, and price information in addition to the basic description of the feature from the feature class table.

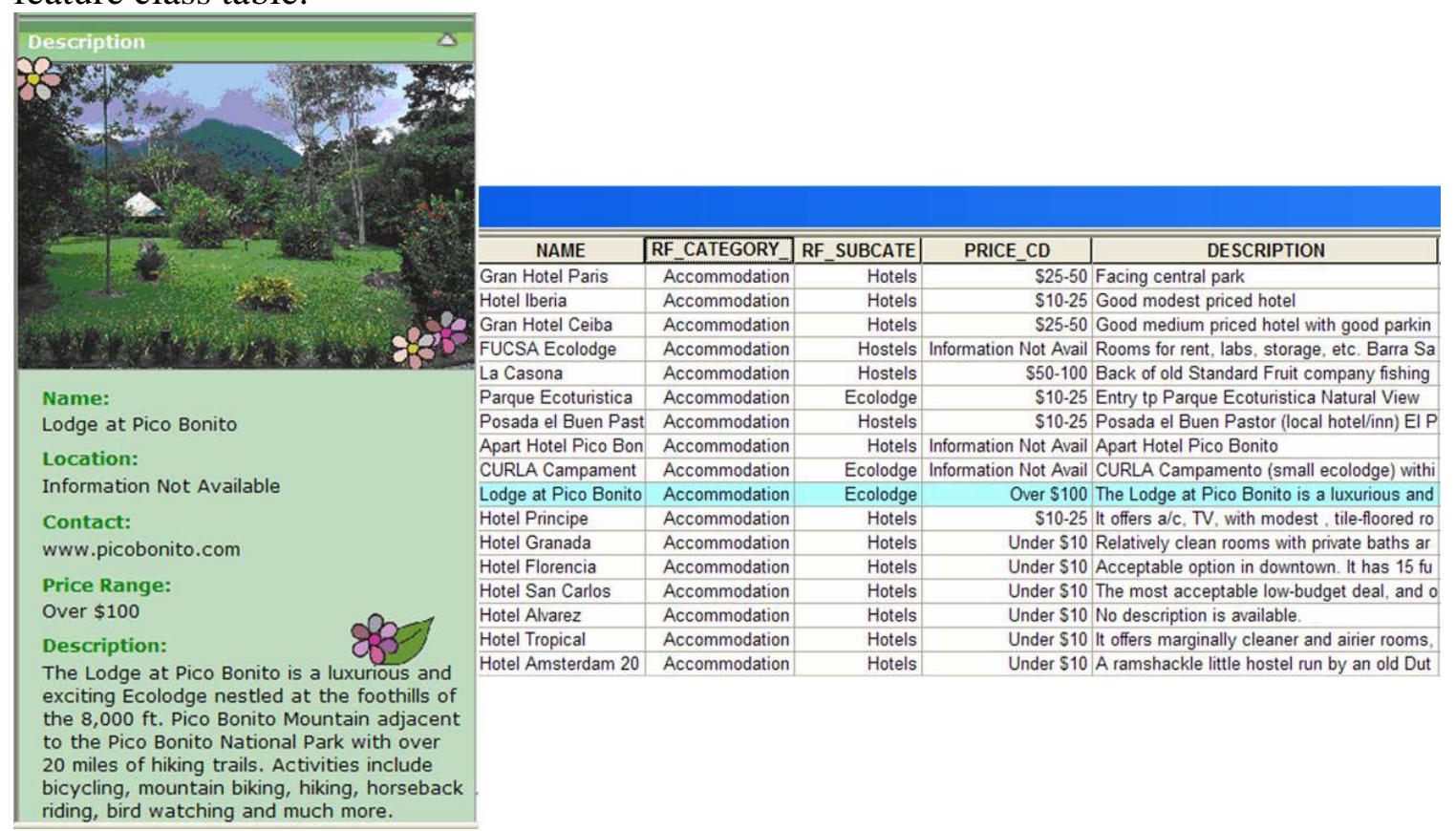

Figure 6-8. Image Hyperlink and Description. 


\subsubsection{Multiple-Select Functionality}

The select multiple features functionality was built to incorporate the ability to select multiple features within the same category. The system displays all the selected features on the map and names of the selected features in the description panel. It also allows potential visitors to select hotels or restaurants that fall within a user-specific price range.

The code below queries all the features specified in the category drop-down list, stores them in a feature graphic layer, and reports the total number of features stored. After features are selected, the map zooms to full extent and displays the total number of selected features and the names of individual ecotourism service locations that were selected.

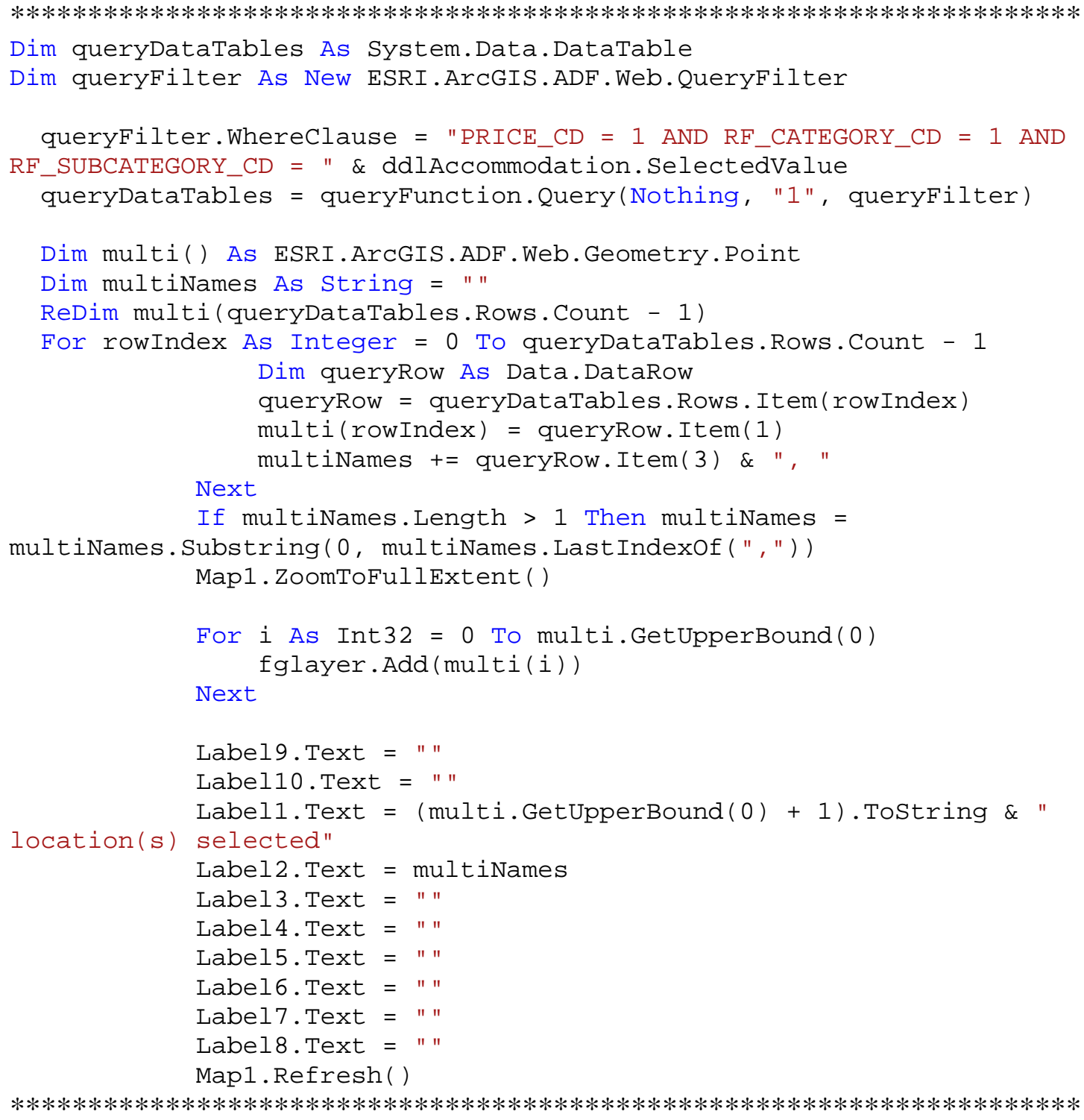


Figure 6-9 illustrates the resulting graphic interface reflecting the VB code discussed above.

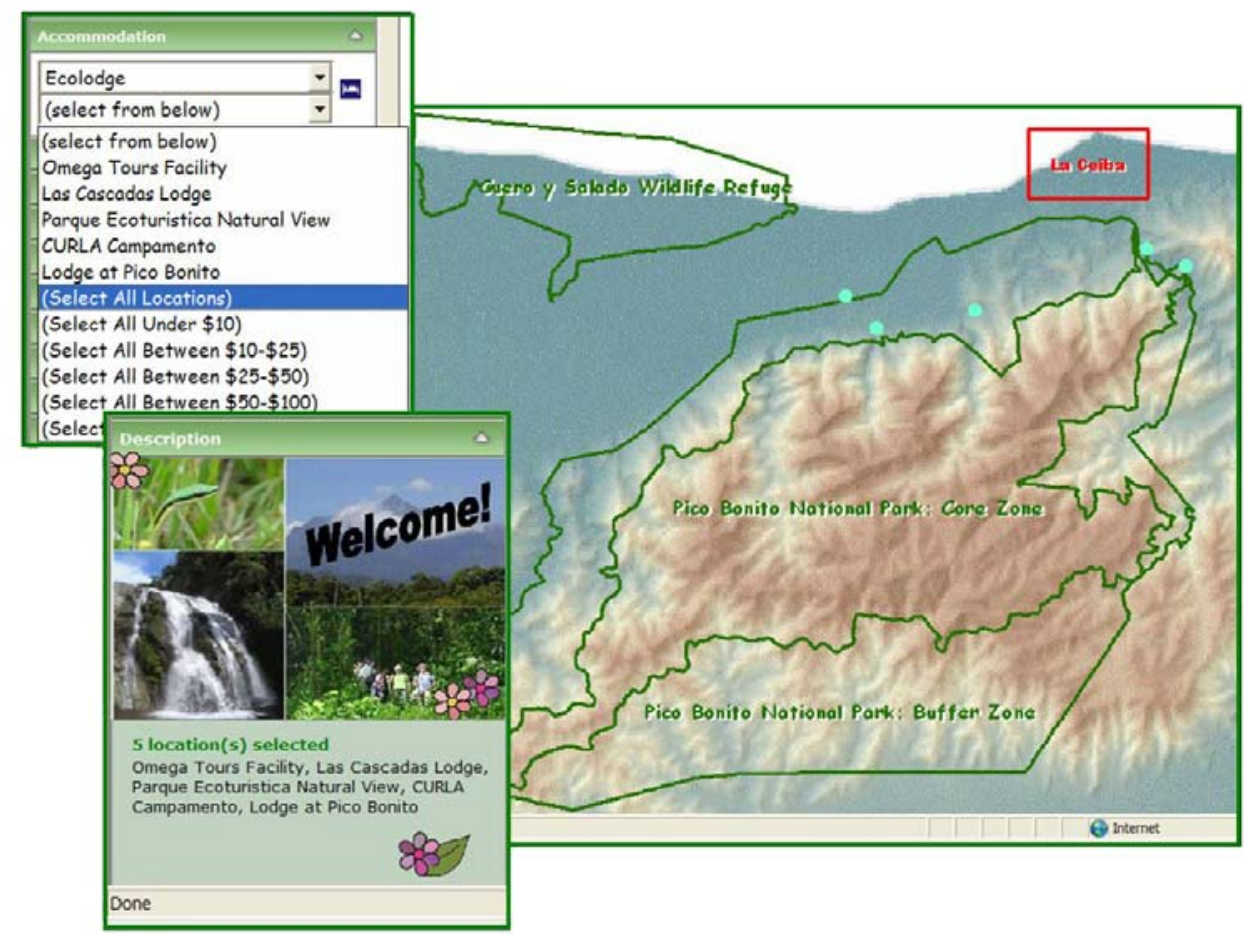

Figure 6-9. Multiple Select Functionality.

\subsubsection{MapTips}

MapTips is an ArcGIS web control that displays information about a point feature by hovering the cursor over or clicking the point feature on the map. This tool was specifically implemented to accommodate the client's request for making the website highly interactive (e.g., clicking on the screen and getting back a pop-up window with information about the point, in return).

Making MapTips work involved three steps. First, a feature class containing the photo points was created. The only points that had associated images in the TourismService_PA feature class were isolated and exported as a new feature class called PhotoPoints. The PhotoPoints layer in the map document was then specified in the MapTips control. In order to display the point-specific image and information, the following code was added into the "Set Body Format String" setting in MapTips (Figure 6-2 (5).

$<$ b $>$ Image Captured: $</$ b $>$ \{DATE_\}

<img src=

'http://localhost/ProtectedAreasTrails/IMAGE/RESIZE/\{JPG_LG\}' />

$<$ b $>$ Image and Description Provided by: $</$ b $>$

$\mathrm{Dr}$. Robert Ford

$<$ b $>$ Image Description: $</$ b $>$

\{DESCRIPTION\} 
Figure 6-10 shows a screenshot of the MapTips.

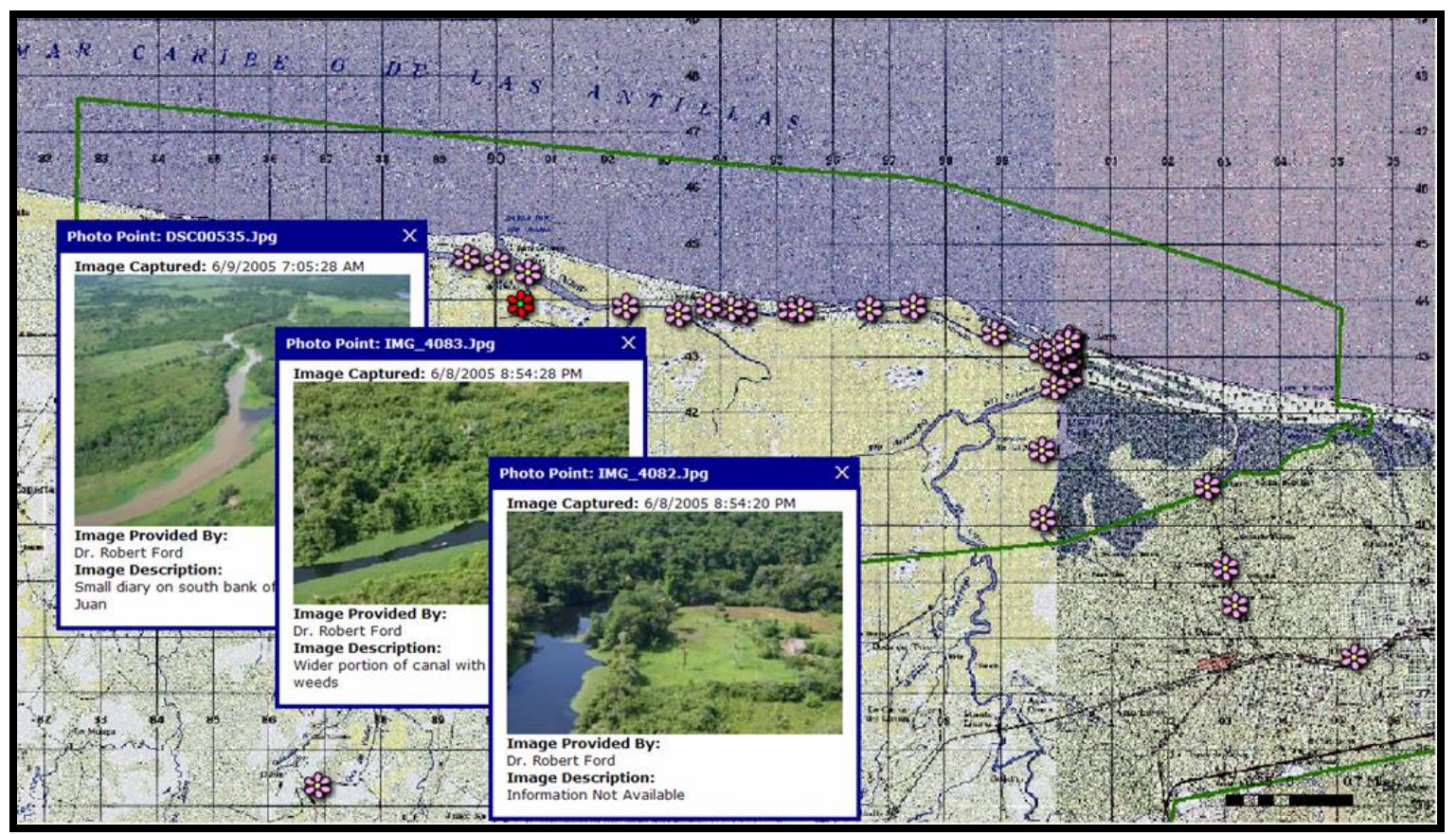

Figure 6-10. MapTips. 


\section{Discussion}

Although some challenges were encountered, the project objectives were successfully fulfilled by (a) developing two prototype websites using ArcGIS Server, and (b) creating a central geodatabase to facilitate data storage, entry, and maintenance in the future.

\subsection{Challenges}

The two most difficult challenges encountered in this project were scarcity of projectspecific data, and implementation of the new technology. Much existing information appeared to be useful at first, but in fact, a significant amount was not applicable for this project due to its lack of metadata, geographic reference, or specific attribute information. Numerous existing data layers were examined in order to determine the final layers to be displayed online. Oftentimes, the same data layers were duplicated in many directories. Similarly, slightly different versions of the same data layers were commonly found. This made it extremely difficult to select the most current and accurate data layers to be distributed online.

Collecting field data overseas was another challenge. A group of field data collectors had to be assembled, then trained to use GPS units to conduct field work. Data collection was further delayed as we had to wait for the end of the rainy season in Honduras in order to capture GPS data. Field data was gathered from January 2007 to April 2007. The time constraint in collecting data was probably the highest hurdle encountered in this project.

One of the challenges of developing a web-GIS application is the constantly changing and improving technology. Developing a web-GIS application with ArcGIS Server 9.2 was a challenge. It was difficult to communicate with the local participants about the project's intent, goals, and scope when all the project participants were learning about the software capabilities on-the-go. However, this project provided all the project collaborators with valuable learning experiences and an understanding of ESRI's newest software products: ArcGIS 9.2 desktop application and ArcGIS Server 9.2.

\subsection{Accomplishments}

This project successfully fulfilled the functional requirements and delivered the following products to the client: the data, a file geodatabase and two prototype websites that distribute spatial information stored in the geodatabase online.

GPS data collected for this project is a great asset for the protected areas. The client now has a collection of data for future use. Approximately 450 point features, 15 trails, 15 access road locations, and 200 images were newly captured by the end of the project. Although not all data was utilized for the web-GIS application, every feature was stored in the geodatabase for future use. As a result, trail maps and other useful tourist guide maps can now be generated using the newly acquired data.

Local park staff and students from local universities were trained to use hand-held GPS units to gather GPS data and associated data for this project. Community participation is a key element to successful ecotourism. Local community involvement was achieved through a collaborative effort in collecting field data. 
The geodatabase integrates data from various sources and centralizes GIS data pertaining to the protected areas. Both vector and raster spatial data utilized in this project are contained in one geodatabase and every data layer has complete metadata. Data layers are categorized by major themes (e.g., transportation, hydrology, cadastral) within the geodatabase for quick access to the feature layers. The geodatabase created in this project is expandable and flexible for future growth. Data maintenance is crucial in providing tourists with the most current and reliable information. The database has a large data capacity and is ready to accommodate new data as needed. The client-specific subtypes and domains are already embedded in the geodatabase, which ensures quick and consistent data entry. The geodatabase is intelligent and flexible to accommodate new domain types. New domains can be added directly in the geodatabase or in Excel, which makes it easier to use, and it can be quickly updated in the geodatabase, if desired. This will also facilitate the responsibilities of the park managers to maintain and update the database and the web-GIS application in the future.

The final outcomes of this project are two prototype websites: the protected areas trails map and the ecotourism services map. The protected areas trails website (Figure 7-1) informs the potential visitors about the natural environment of the protected areas (e.g., ecosystems, forestry, soil type) including the trail locations. This website utilizes the ArcGIS Web Mapping Template and uses default settings and basic configurations. It has no custom Visual Basic code. Although it is very simple to create, the website has only minimum functionalities embedded in it.

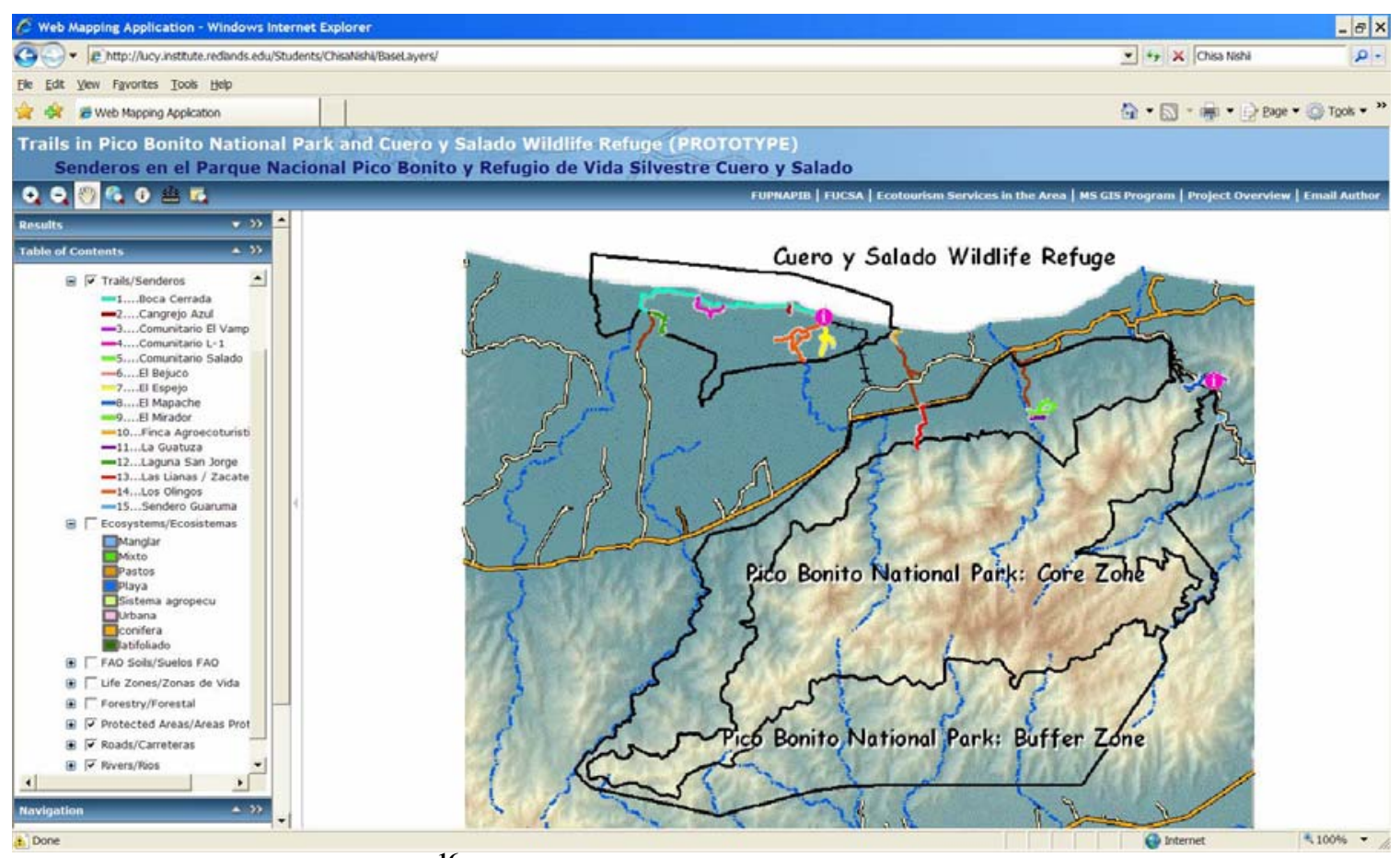

Figure 7-1. The Trails Website ${ }^{16}$.

The ecotourism services map, on the other hand, informs the potential visitors about the location of trails, restaurants, souvenir shops, museums, and other ecotourism service

\footnotetext{
${ }^{16}$ The Protected Areas Trails Map is available at: http://lucy.institute.redlands.edu/Students/ChisaNishii/ProtectedAreasTrails
} 
locations around and within the protected areas (Figure 7-2). The website allows tourists to access specific information and images associated with each tourism service they select from the custom drop-down lists. The Internet audience can directly interact with the web-GIS site by not only panning around the map and zooming in and out as they wish, but also by clicking on the map for image and information retrieval. This website supports the decision-making process of the potential tourists as to where to stay overnight or dine by allowing users to select hotels or restaurants under the user-specified price range.

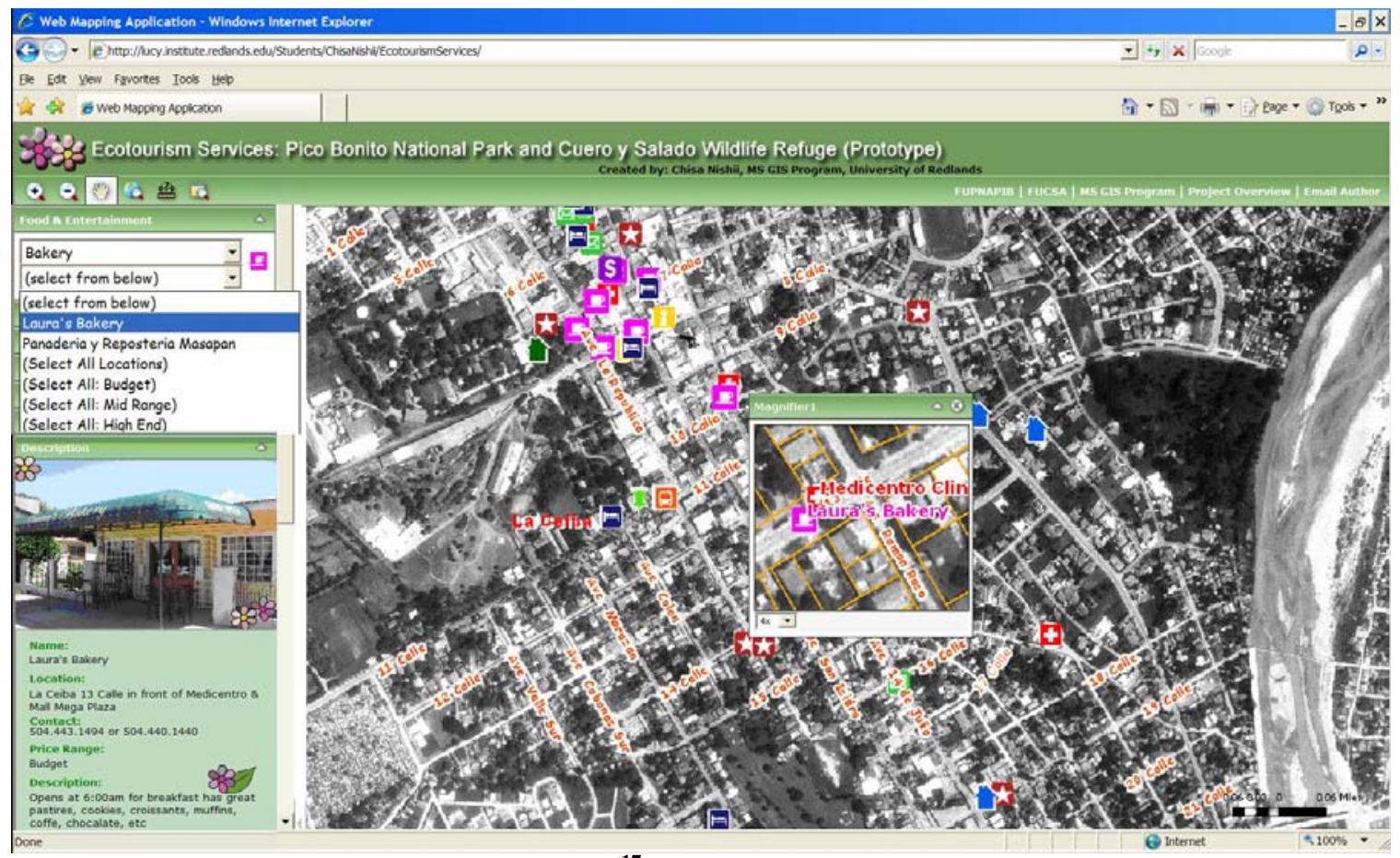

Figure 7-2. The Ecotourism Service Website ${ }^{17}$.

\subsection{Implementation}

This project is a preliminary study leading to the actual implementation of the website. The objective of creating two sample websites was to provide the client and the project participants with the ability to conduct a cost-benefit analysis for implementing a website using the ArcGIS Server technology. There are several considerations when deciding whether to implement the prototype web-GIS application in the protected areas: cost, installation, and whether to customize the websites.

\section{Cost:}

As previously mentioned, the protected areas are chronically underfunded and purchasing necessary equipment for building a web-GIS application imposes further financial burdens on the protected areas' administrative organizations. Costs associated with the development of the web-GIS application include, but are not limited to: (a) purchasing

\footnotetext{
${ }^{17}$ The Ecotourism Services Map is available at: http://lucy.institute.redlands.edu/Students/ChisaNishii/EcotourismServices
} 
ArcGIS 9.2 desktop and ArcGIS Serve 9.2 licenses, (b) training staff in ArcGIS 9.2 usage and data collection, (c) purchasing proper hardware (e.g., computers and equipment for data collection), and (d) maintenance fees (staff and resources). Specific pricing information on purchasing GIS software can be obtained by contacting one of the ESRI representatives ${ }^{18}$ or ESRI's business partners ${ }^{19}$.

\section{Installation}

Web-GIS using ArcGIS Server is a fairly new technology. Various organizations have expressed their interests in this web-GIS application. However, most Honduran organizations interested in this Web Application use ArcView 3.3. Because building a Web GIS application requires proper equipment, obtaining an ArcGIS 9.2 and ArcGIS Server license are the first considerations for the project partners. Furthermore, no formal decision has been made in regards to who will host and maintain the system in the long term. The websites can be installed once the project partners decide who will maintain the system. An installation guide (APPENDIX J) was created for future installation of the two websites. Some data used for this project was derived from books (e.g., Lonely Planet and MOON) and the Internet (e.g., DISEÑARTE), and proper permission must be obtained prior to officially distributing data online.

\section{Default vs. Custom Web-GIS Application}

The default website comes with a set of advantages and disadvantages, but the same can be said for the customized website. The default website is simple to create but less intuitive and user-friendly. The custom website, on the other hand, has more tools and functionalities, and it is easy for non-GIS users to navigate around the website, but requires more time to build, and a staff or contractor with programming skills. The custom web-GIS application in this project required one year to develop, including the data acquisition and geodatabase design that were integral parts of the web-based GIS application. Comparing the two websites allows the project partners to determine whether the ArcGIS Server default website is satisfactory, or if creating a custom website is worth pursuing.

\footnotetext{
${ }^{18} \mathrm{http} / / /$ www.esri.com/software/arcgis/arcgisserver/how-to-buy.htm

19 http://www.esri.com/partners/index.html
} 


\section{Future Recommendations and Conclusion}

There is a great potential for improved online mapping display, performance, and functionality. Data collection can be expanded to provide tourists with more information about features of the protected areas, such as tours and activities, bird watching sites, and flora and fauna information. More attractive and useful information to tourists includes location of tourist-friendly neighborhoods. Obtaining detailed directions to navigate from one area to another is a functionality that can be added using the network analyst extension in ArcGIS Server.

The two prototype websites were made available around the globe to numerous people with varied GIS experiences as well as varied computers and Internet connections for testing purposes. This provided a good opportunity to determine (a) how the website performs when accessed from overseas, and (b) how useful and intuitive the websites are.

The prototype websites were viewed by the following parties:

- Project client in the USA

- Project partners in Honduras

- Family and friends from Guatemala and Japan

- GIS domain experts from ESRI

Their feedback on the website became helpful and useful in defining future website improvements. Successful web-GIS application involves comprehensive design and reasonable functionality. The most common suggestions for improved design was to make the image and description of individual point features more visible to the viewers. This can be accomplished by moving it to the top of the page, or making it expandable to the side of the page, rather than keeping it on the bottom of the screen. Second, the author was informed that the websites are not viewable while using web browsers such as Mozilla Firefox. Making the websites compatible with different web browsers is recommended in the future. Finally, the speed of data display was addressed. Internet connection in Honduras is slow and enhanced website performance will be required in the future.

Organizing and conducting quality control over existing GIS data is also recommended. Base layers utilized in this project were considered adequate for the purpose of this project, but proper data organization and management is recommended for a more effective use of spatial data in the future. Currently, there are no data quality control measures implemented in Honduras. There is no metadata documentation. There are multiple versions of the same layers and no one knows which one is the most current. Organizing existing data may require some courage to discard "valuable" data that was collected from Honduras over time. However, this will not only eliminate clutter, but will also lead to a more efficient data retrieval system and an effective use of the data in the long run. There are workshops at ESRI on Data Quality Assurance and Quality Control. Basic training and understanding about data maintenance is highly recommended for the future GIS data management for the protected areas in Honduras. 
This prototype project implemented the ArcGIS 9.2 desktop application and ArcGIS Server 9.2 to create two prototype websites to explore future tourism services, and a geodatabase to facilitate data entry and future data maintenance.

Overall, this prototype project demonstrated the possibilities for web-based GIS technology using ArcGIS tools to promote ecotourism and assist park management in the protected areas. Successful ecotourism involves several complex ingredients, including the natural environment, the local communities, and the tourism system. This project complies with goals of achieving a "mutually supporting partnership" among those elements by promoting tourism in the protected areas by distributing tourist information online, encouraging local community participation in the project, and supporting the future natural resource management in the area.

Effective advertisement through promotional materials via magazine advertisement, paper flyers, and Internet sites are crucial for attracting both domestic and international tourists in the area. This project demonstrated that web-GIS applications show great promise in promoting ecotourism, both by distributing tourist information online and assisting the tourists' decision-making process in regards to where to visit while they stay in the protected areas. The local community involvement was achieved through a collaborative effort in collecting data and designing a geodatabase. It is the author's hope that the future data and website maintenance will assist the protected areas' administration teams with more efficient and effective tourism planning and natural resources management.

This project is a first step, leading the way for the protected areas management organizations to further develop web-GIS applications using the ArcGIS Server technology. It has been the author's privilege to have added to the protection and future management of the protected areas in northern Honduras, and collaborated with the local communities in Honduras and the GIS program at the University of Redlands. It is rewarding to work with ideas, technology, and individuals who are making a difference. 


\section{References}

Arctur, D., \& Zeiler, M. (2004). Designing geodatabases: Case studies in GIS data modeling. Redlands, CA: ESRI.

Avila, J. (2004). Estrategia nacional de Ecoturismo: Formulacion y armonizacion de la estrategia metodologica para el desarollo del ecoturismo en Honduras.

Tegucigalpa, Honduras: Instituto Hondureno de Turismo (IHT).

Balram, S., \& Dragiâceviâc, S. (2006). Collaborative geographic information systems. Hershey, PA: Idea Group Pub.

Bearly, T., Theobald, D., Hobbs, T., \& Zack, J. (1998). Disseminating Natural Diversity Information using ArcView IMS: Design Issues and Technical Considerations. Proceedings of the 1998 Annual ESRI User Conference. Retrieved November 10, 2006 from http://gis2.esri.com/library/userconf/proc98/PROCEED/TO200/PAP184/P184.htm

Boers, B. (2005). Sustainable tourism infrastructure planning: A GIS-based approach. Proceedings of the 2005 Northeastern Recreation Research Symposium. Retrieved October 23, 2006, from http://www.treesearch.fs.fed.us/pubs/22288

Boo, E. (1990). Ecotourism: The potentials and pitfalls. Washington, DC: World Wildlife Fund.

Buckley, A. (2007). Creating a multidirectional oblique weighting (MDOW) hillshade. Retrieved July 23, 2007 from http://www.institute.redlands.edu/Users/aileen_buckley/Basemap/labs/elevation.h $\underline{\mathrm{tm}}$

Case, T. R., \& Baker, T. R. (2003). Looking at student-collected global warming data using ArcIMS. Proceedings of the 2003 Annual ESRI User Conference. Retrieved October 23, 2006, from gis2.esri.com/library/userconf/proc03/abstracts/a0587.pdf

Chandler, G., \& Prado, L. (2007). Lonely Planet Honduras \& the Bay Islands. Singapore: Lonely Planet Publications.

ESRI (2005). Cartography with ArcGIS. Course Lectures.

ESRI (2006a). Introduction to ArcGIS Server. Course Lectures.

ESRI (2006b). Building Geodatabases. Course Lectures.

ESRI (2007a). ArcGIS Server Manager Help (Version 9.2) [Computer software]. Redlands, CA:

ESRI (2007b). ArcGIS Server 9.2 web mapping application compared to ArcIMS 9.2 viewers. Retrieved July 2007 from http://www.esri.com/software/arcgis/arcgisserver/about/literature.html

Humphrey, C. (2006). Moon Honduras. Emeryville, CA: Avalon Travel Publishing.

The International Ecotourism Society (2007). Ecotourism. Retrieved July 8, 2007, from http://www.ecotourism.org/ 
IUCN: The International Union for the Conservation of Nature (2006). Ecotourism. Retrieved October 13, 2006, from http://www.iucn.org/themes/spg/Files/beyond_fences/bf_section4_6.html

Jensen, D., \& Stonich, T. Parks \& recreation information system - Integrated ArcIMS Application, Boulder, Colorado. Retrieved June 20, 2007 from gis2.esri.com/library/userconf/proc05/papers/pap1272.pdf

Knox, D. (2004). 3D terrain visualization and virtual fly-through for tourism conservation using geographic information systems (GIS). Retrieved October 23, 2006, from http://72.14.253.104/search?q=cache:zzsl34R2esJ:eprints.usq.edu.au/archive/00000041/+Donna+Knox+3D\&hl=en\&ct=clnk\&cd $=5 \& g l=u s$

Pico Bonito National Park: Hiking in the rain forest. (n.d.). Retrieved July 15, 2007, from http://www.picobonito.com/PB_files/act.html

Tang, W., \& Selwood, J. (2003). Connecting our world: GIS web services. Redlands, CA: ESRI Press.

Vreugdenhil, D. X., House, P.R., Cerrato, C.A., Matrinez, R.A., \& Pereira, A.C. (2002). Rationalisation of the protected areas system of Honduras Volume 1: Main study. Retrieved October 19, 2006, from http://resweb.llu.edu/rford/ESSE21/LUCCModule/docs/Protected_Areas_Main_S $\underline{\text { tudy.pdf }}$

Weaver, D. B. (1998). Ecotourism in the less developed world. Oxon; New York: CAB International.

Wylie, J. (2001). Water trails nature tourism and interpretation at the Cuero y Salado Wildlife Refuge, Honduras. USDA Forest Service, Honolulu, Hawaii, USA. 


\section{Appendix A - VBA Code: Microsoft Office Excel Data Catalog Utility Tool}

The VBA code in this appendix creates a catalog of all the objects in the user-specified directory, including the full path name of the object, name, and type. Additional information extracted from the object include number of rows, projection, and feature type, if applicable. The full appendix, containing approximately 350 lines, appears on the enclosed project CD: MIP_DataInventory.xls

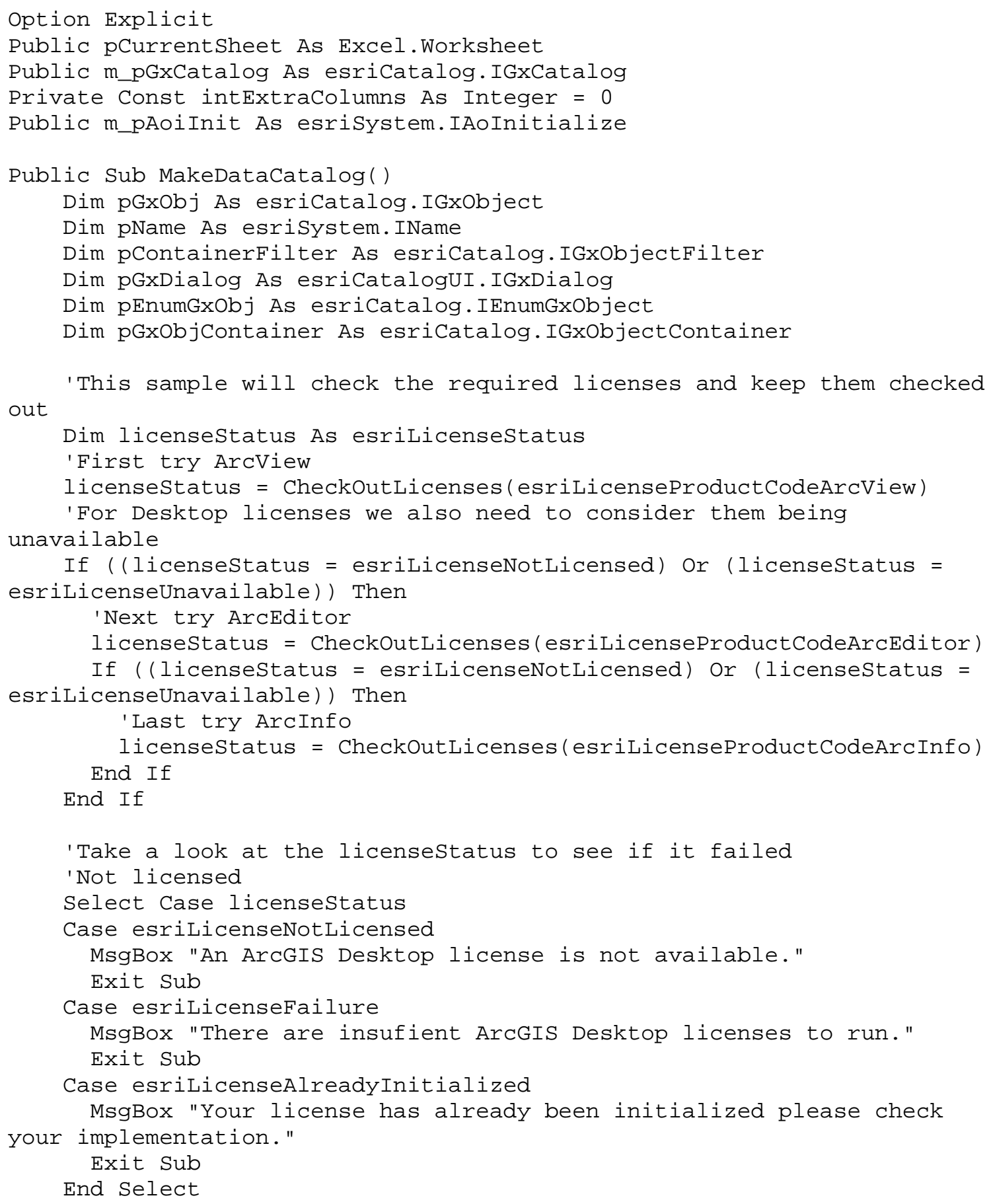




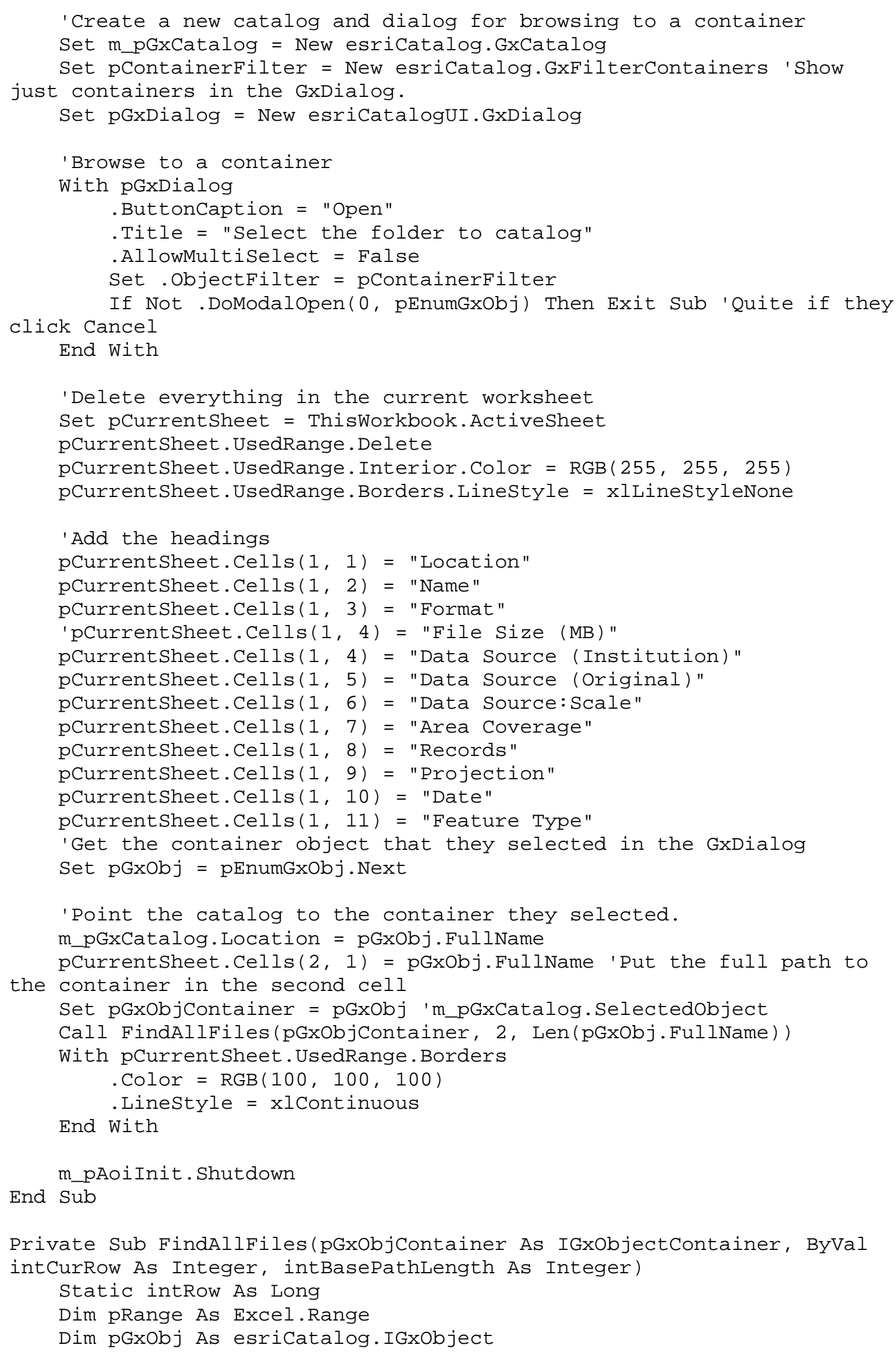


Dim pGXFolder As esriCatalog. IGXFolder

Dim pGXDataset As esricatalog.IGXDataset

Dim pGXFileFilter As esriCatalog.IGxFileFilter

Dim pEnumGxObj As esricatalog. IEnumGxobject

Dim pGxObjProps As esricatalog.IGXObjectProperties

Dim pRasDataset As esriGeoDatabase.IRasterDataset

Dim pRasBandCol As esriDataSourcesRaster. IRasterBandCollection

Dim pName As esrisystem. IName

Dim pTable As esriGeoDatabase.ITable

Dim strFolderText As String

Dim strFileExt As String

Dim intFillFColorIndex As Integer

Dim intStarRow As Integer

Dim dblSize As Double

'Added on 1/15/2007 with Robert B. and Mike C.'S assistance $<<<<<<~$ Dim pGXDatabase As esriCatalog. IGXDatabase2

Dim pGXFeatureClass As IFeatureclass

Dim pGeoDataset As IGeoDataset

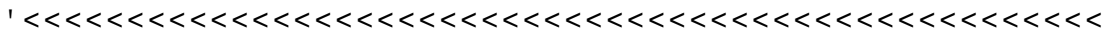

Dim strPrjFileName As String

Dim strPrjPathName As String

Set pGxFileFilter = m_pGxCatalog.FileFilter

intRow = intCurRow

'Get all the children objects from the container

Set $p E n u m G \times O b j=p G \times O b j$ Container. Children

If pEnumGxObj Is Nothing Then Exit Sub 'If there are no children then bail out of the routine

'Get all the cells from the spreadsheet

Set pRange $=$ pCurrentsheet. Cells

Set $\mathrm{pG} \times 0 \mathrm{Obj}=\mathrm{pG} \times 0 \mathrm{bjContainer}$

'Strip the base path off of the full path.

strFolderText $=" . \backslash " \&$ Mid (pGxObj. FullName, intBasePathLength +2$)$

'Oscialte the fill color between containers

If pRange.Cells(intRow, 1). Interior.ColorIndex $<36$ Then

Else intFillFColorIndex $=36$

End If

intFillFColorIndex $=34$

intstarRow $=$ intRow +1 'Iterate the row

Set $p G \times O b j=$ pEnumGXObj. Next 'Get the first object in the container.

Do Until pGxObj Is Nothing

If Not Typeof pGxObj Is IGxFolder Then 'Skip all folders. intRow = intRow +1 'Iterate the spreadsheet row. 'pRange (intRow, 1) = strFolderText 'The modified path pRange (intRow, 2) = pGxObj. Name 'The name of the object. pRange $($ intRow, 3) = pGxObj.Category 'The type of object.

This can be overwritten later.

pRange (intRow, 4) = "MIRA"

pRange (intRow, 6) = "UNK"

'pRange (intRow, 4) = "N/A" 'Add N/A to the cell for size

and then overwrite later if appropriate 
pRange (intRow, 8) = "N/A" 'Add N/A to the cell for \# rows

and then overwrite later if appropriate

' $<<<<<<$ Added on 1/15/2007 with Robert B and Mike C's assistance $<<<<<$ pRange (intRow, 1) = pGxObj.FullName

strPrjPathName $=$ Mid $($ pGxobj. FullName, 1,

Len(pGxobj.FullName) - Len(pGxObj. Name))

'If Typeof pGxObj Is IGxObjectProperties Then

' Set pGXObjProps = pGxObj

' 'Get the file size from the object.

' dblSize =

pGxObjProps.GetProperty ("ESRI_GxObject_FileSize")

' If dblSize $>-1$ Then 'The file size is -1 if it can

not be determined otherwise it is the size of the file.

1048576 bytes in a MB. dblSize $=$ Round $((d b l S i z e / 1048576), 3)$ 'There are cell. pRange (intRow, 4) = dblSize 'Put the size into the

' End If

'End If

Dim unknownobject As Variant

Dim indexcounter As Integer

Dim cellCounter As Integer

Dim propName As String

Dim propValue As Object

cellcounter $=9$

'If Typeof pGxobj Is IGxObject Then

Set pGxObjProps $=$ pGxObj

For indexCounter $=0$ To pGxobjProps.Propertycount - 1

propName, propvalue) Call pGxobjProps.GetPropByIndex(indexCounter,

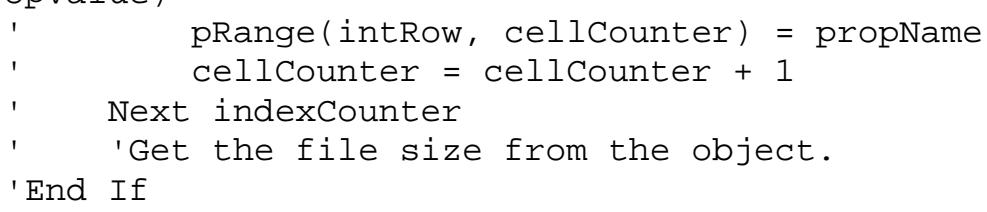

Set pName $=$ pGxobj. InternalobjectName

If Not pName Is Nothing Then

'The name object blow up when opening some datasets so

just ignore errors

'and leave the default type info in the spreadsheet.

On Error Resume Next

If Typeof pName Is ITableName Then 'If it is a table

then open it and get it's number of rows.

Set pTable = pName. Open

If Not pTable Is Nothing Then

'Add the number of rows to the spreadsheet. pRange (intRow, 8) =

FormatNumber (pTable.RowCount (Nothing), $\odot$, vbTrue, vbFalse, vbTrue)

Set pGxObjProps $=$ pGxObj

pRange (intRow, 10) =

pGxObjProps.GetProperty("ESRI_GxObject_FileTime")

Set pGXFeatureclass = pTable

Select Case pGXFeatureClass. ShapeType

Case 1 
pGXFeatureClass. ShapeType

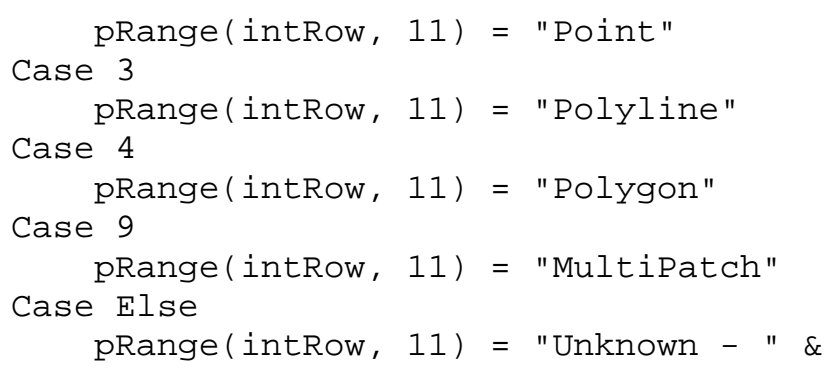

pGXFeatureClass. ShapeType ) End Select

'MsgBox ("ShapeType: " \&

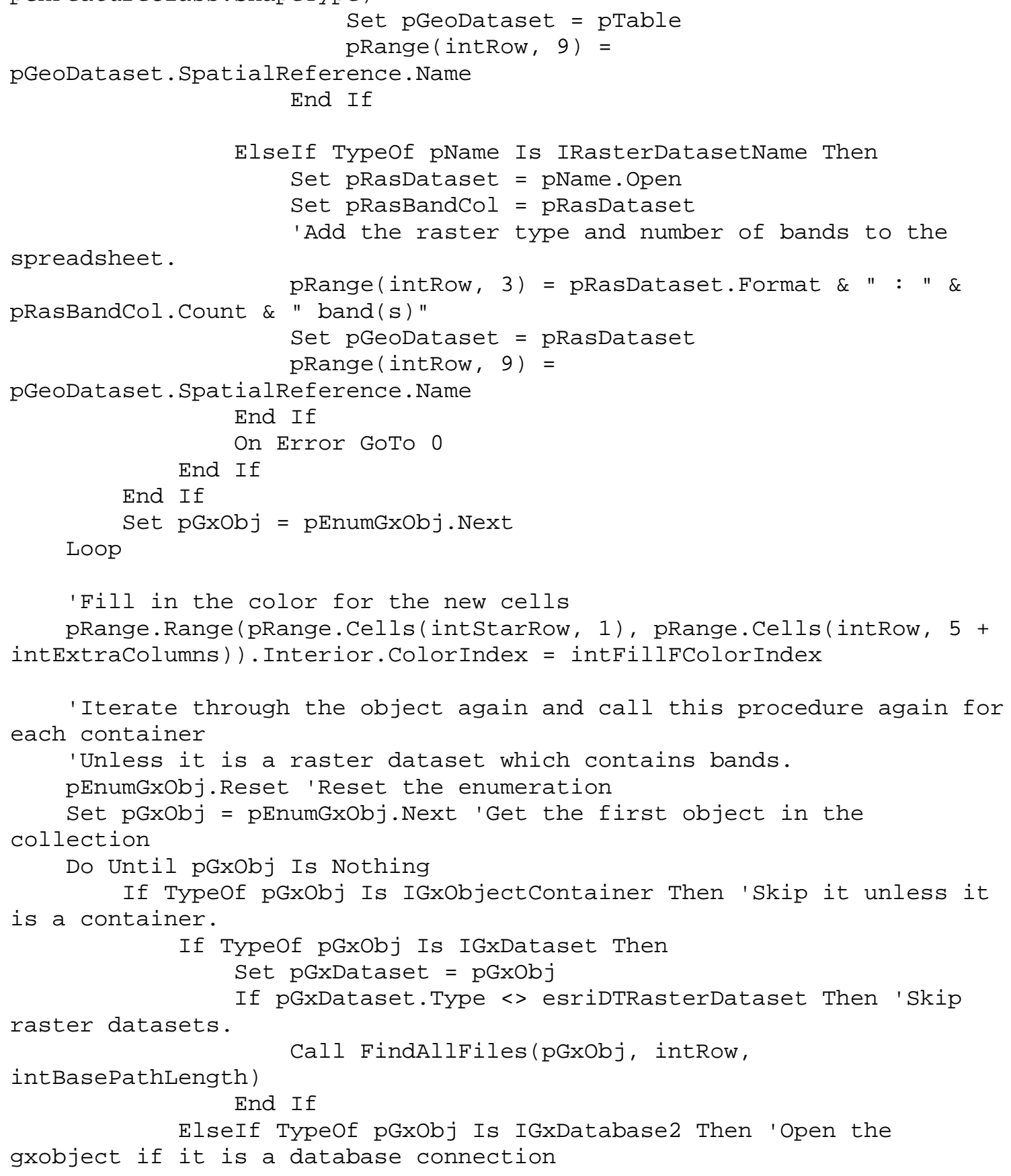




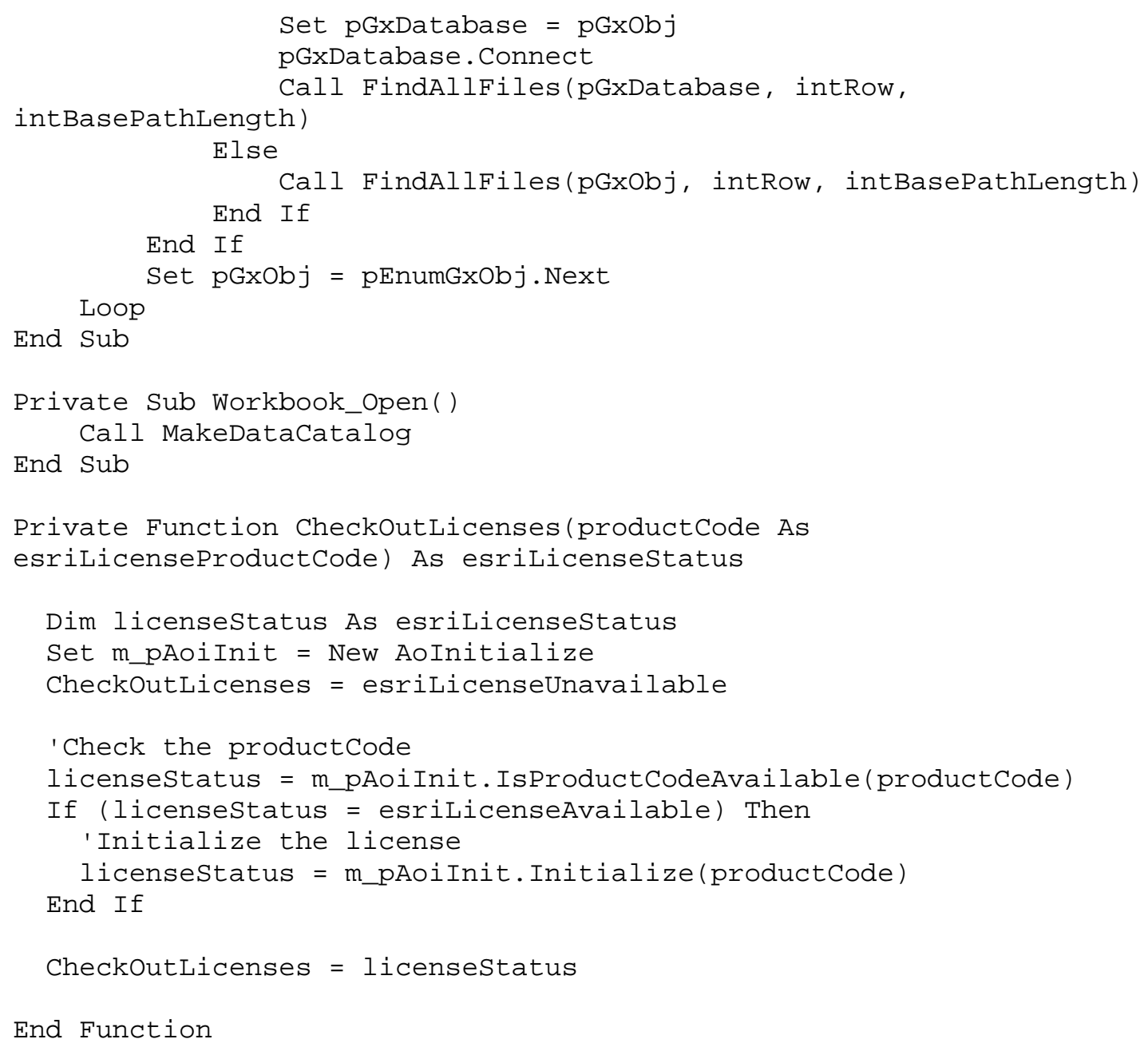




\section{Appendix B - Configuring the web.sitemap to Add External Links}

The configuration code in this appendix adds external links to the webpage. The full appendix appears on the enclosed project CD: EcotourismServices web.sitemap

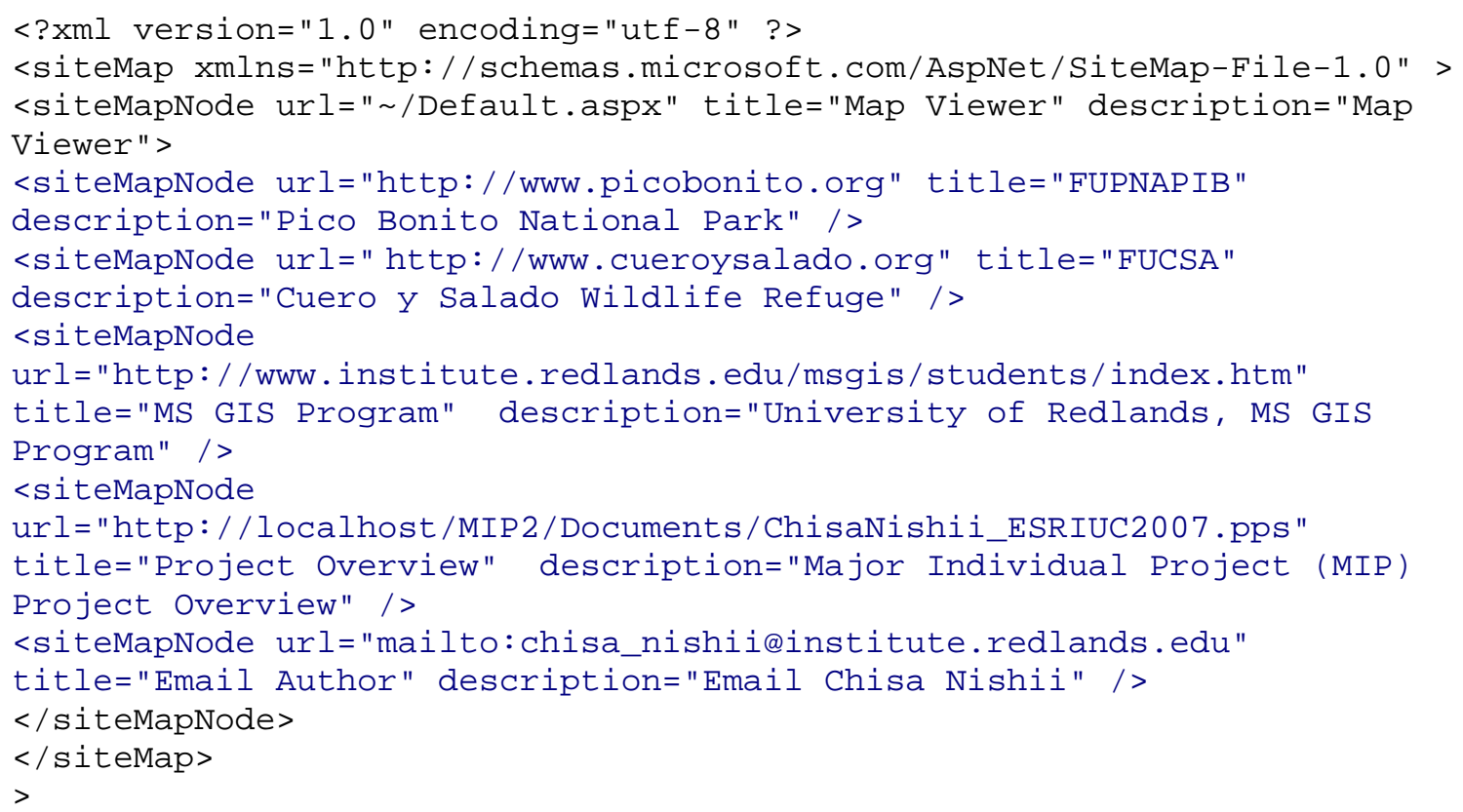





\section{Appendix C - Power Point Slide Show: Web-User Manual}

The power point slide show (containing 12 slides) in this appendix is the user guide of the webpage that is accessible from the website. The full appendix appears on the enclosed project CD: UserGuide.pps
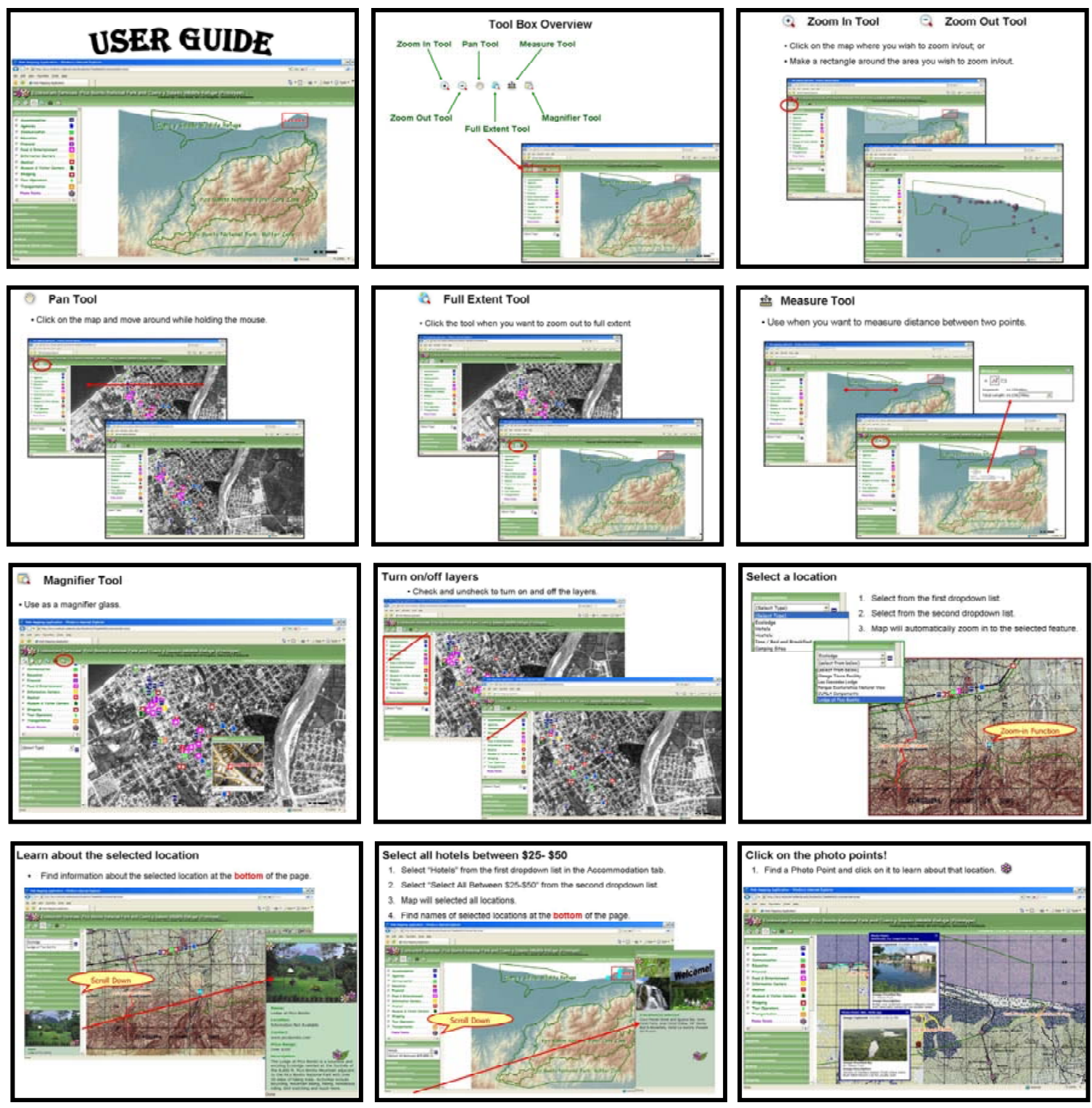



\section{Appendix D - Power Point Slide Show: Project Overview}

The power point slide show (containing 28 slides) in this appendix is the overview of this project that is accessible from the website. The full appendix appears on the enclosed CD: ChisaNishii_ESRIUC2007.pps
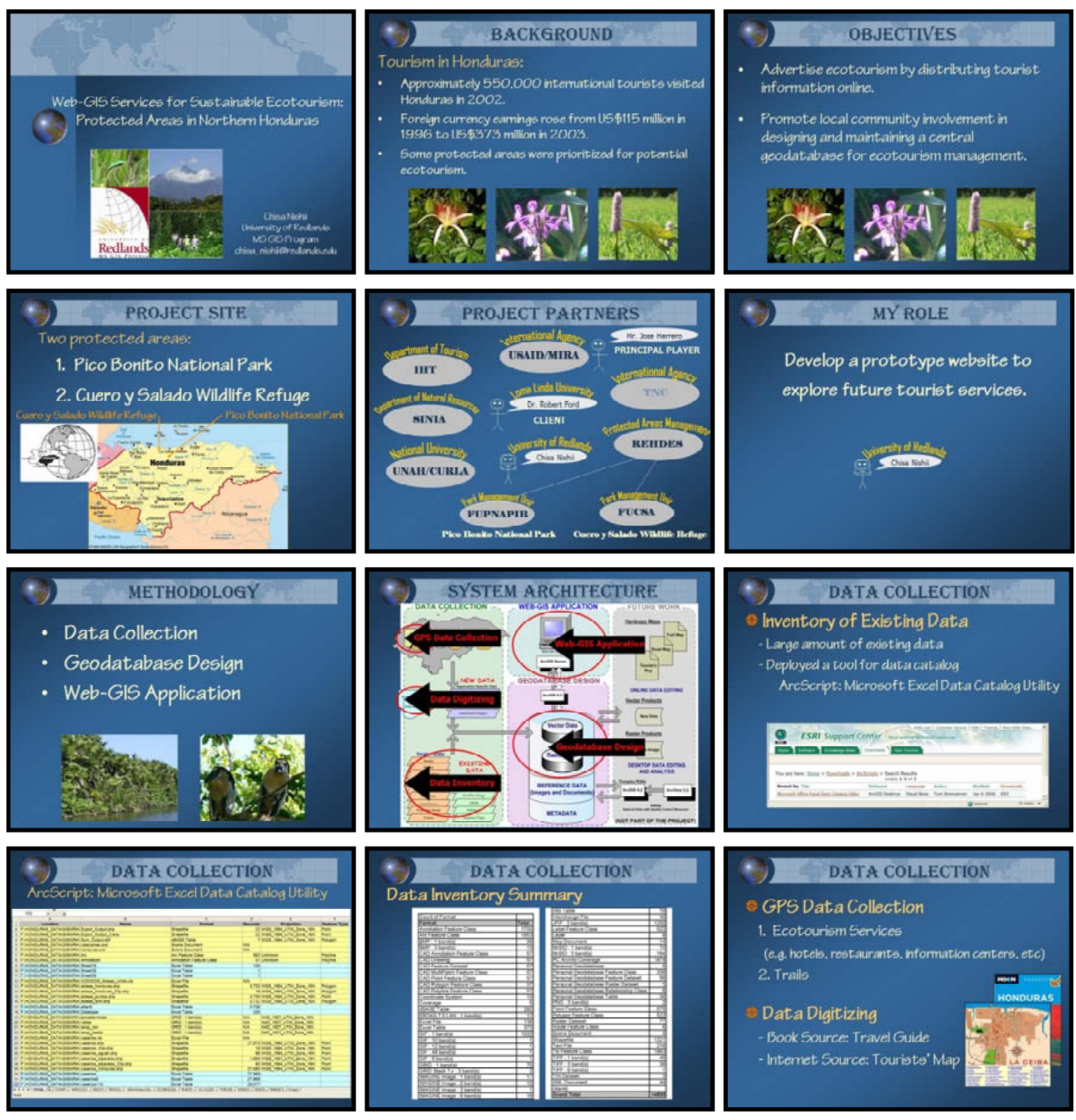

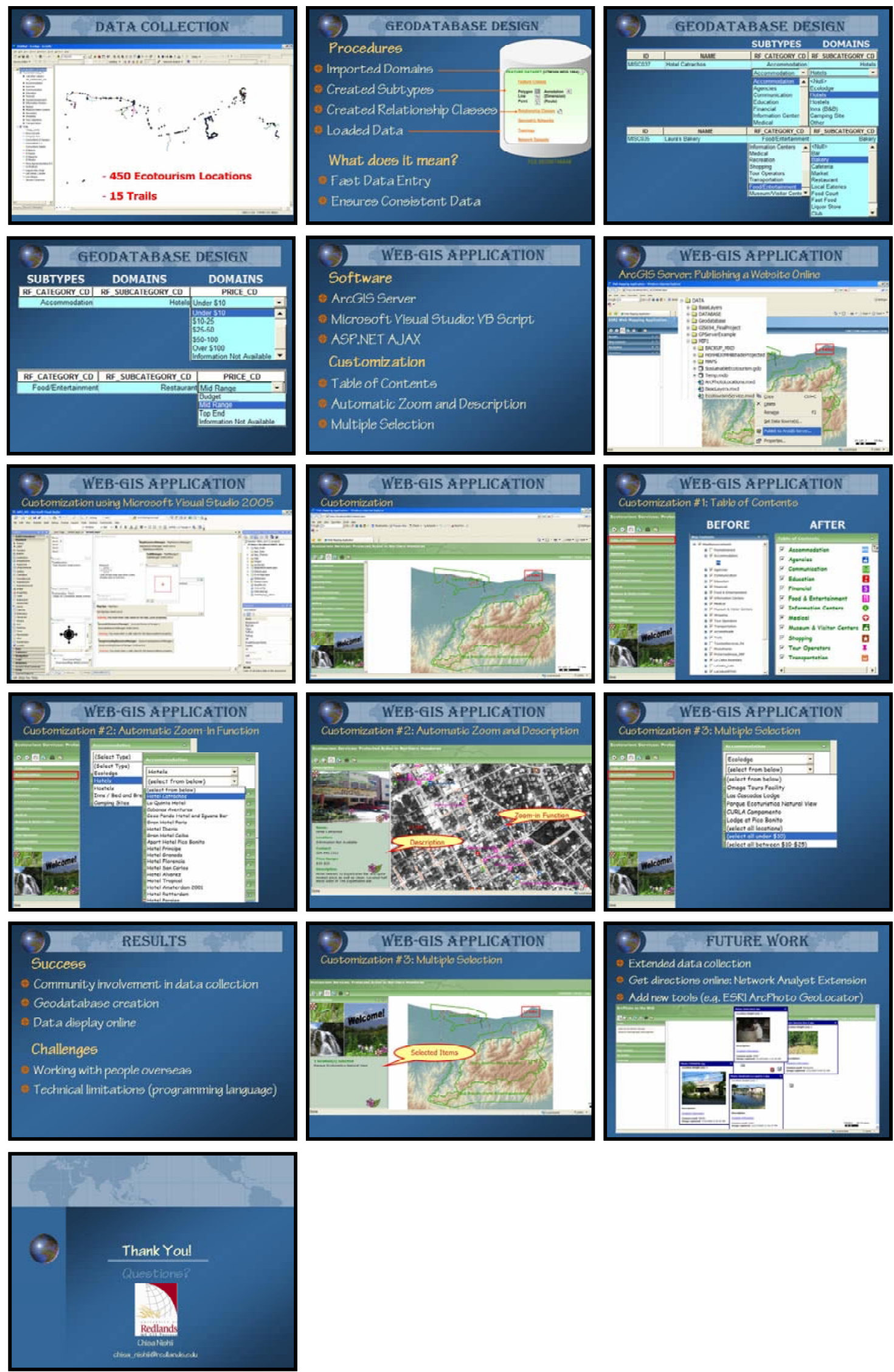


\section{Appendix E - AJAX Enabled Custom Website Template Code: web.config}

The code in this appendix adds the AJAX control to the webpage. The full appendix appears on the enclosed CD: EcotourismServices web.config

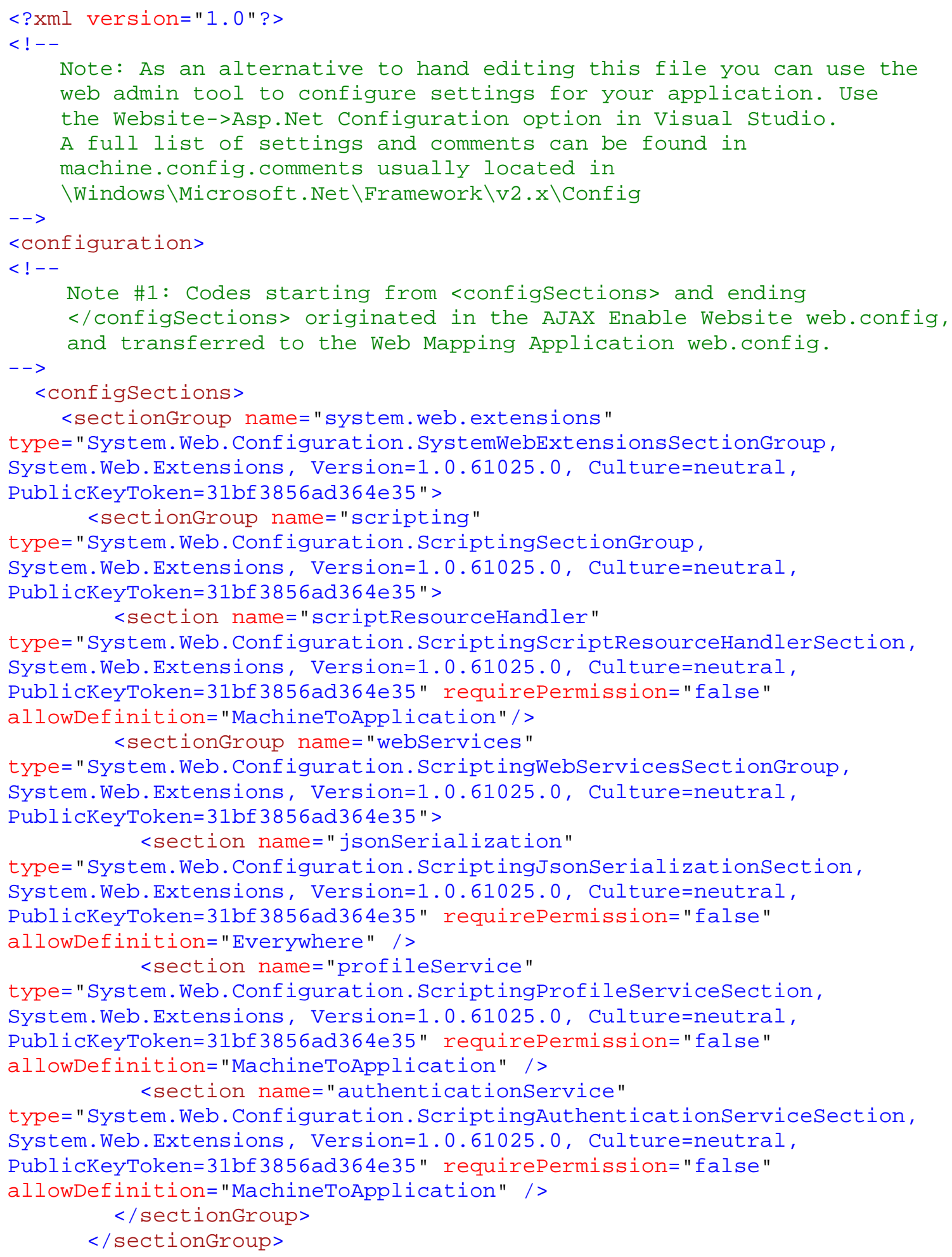




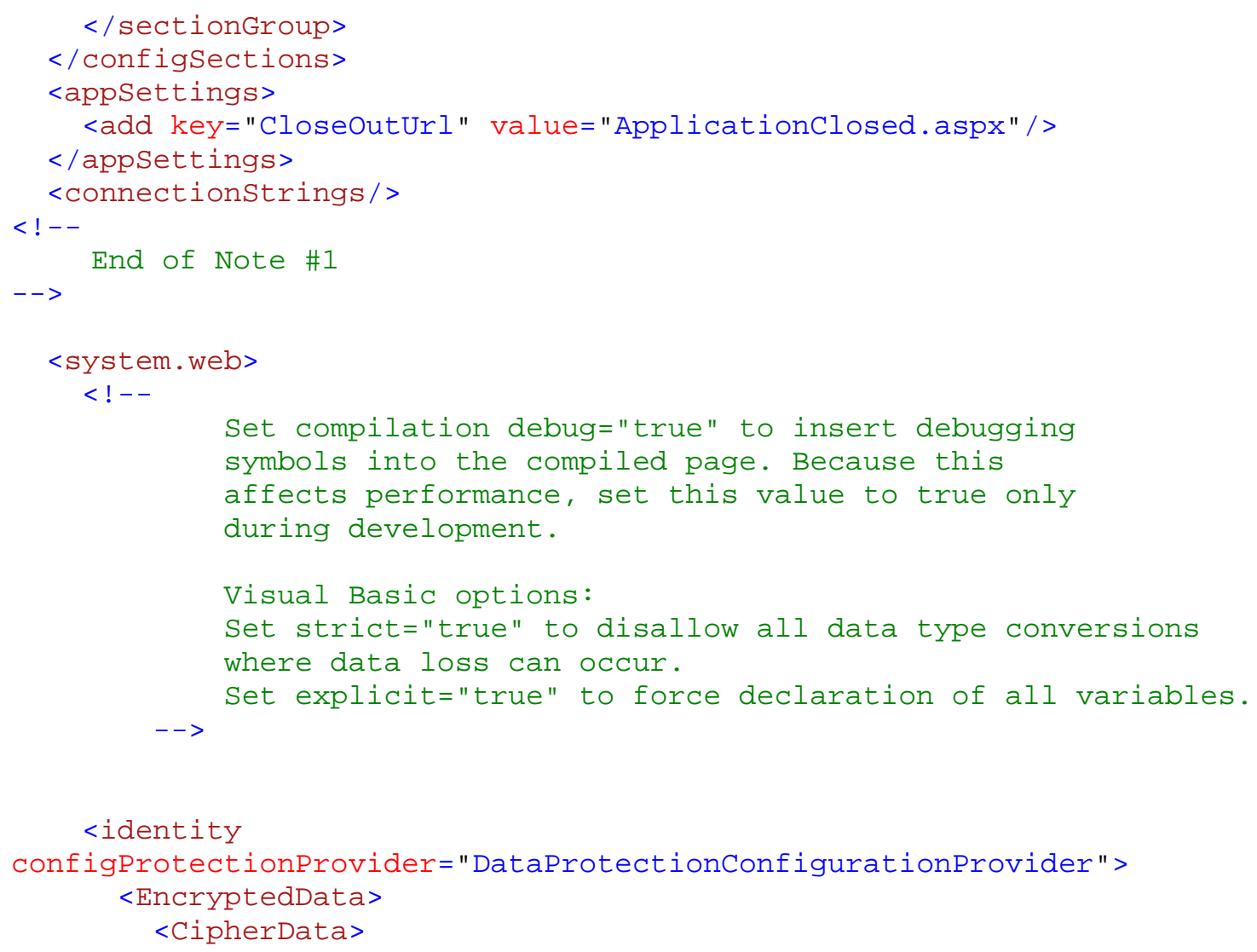

<CipherValue>AQAAANCMnd8BFdERjHoAwE/Cl+sBAAAAOV/y0GzlHE69LmbGic1S6gQAAA ACAAAAAAADZgAAqAAAABAAAABGPiQUiKV26eDlYMqoVr t IAAAAAASAAACgAAAAEAAAAPUnA 3Gtc5wqRBccp+QEFp+WAAAA1X5Ur kNLwMxcqR262ANi2iinBK7slpgSTzu9icAcjydj 7Eri YBhm0xUYo4YcrTxNrBjzuPy3QpyCYRP+0h+NdCbUKWzJX17yTtz6b3CxGCic4t+8F26yG+L Q9AnTNGA61gzTYZPffqWSojK1VWCPCW6rk54qiCFuOWMj fmtxQuHs0jR+t1z80V/541D7Su jVAX481iFsaIl/VhzUrsOmsvZtno6BMxmBvtNeqK9mcpUUAAAAKmGgNUzfIekZqm3eEDRNs jXIOkc=</CipherValue $>$ $</$ CipherData $>$

$</$ EncryptedData $>$

$</$ identity $>$

<compilation debug="true" strict="false" explicit="true"> $<$ assemblies>

<add assembly="System.Web.Extensions, Version=1.0.61025.0,

Culture=neutral, PublickeyToken=31bf3856ad364e35"/> $<$ add assembly="ESRI . ArcGIS . ADF . Web. UI. WebControls,

Version=9.2.1.1332, Culture=neutral, PublickeyToken=8FC3CC631E44AD86"/> <add assembly="ESRI. ArcGIS.ADF.Web, Version=9.2.1.1332,

Culture=neutral, PublickeyToken=8FC3CC631E44AD86"/> $<$ add assembly="ESRI. ArcGIS.ADF, Version=9.2.1.1332,

Culture=neutral, PublickeyToken=8FC3CC631E44AD86"/> $<$ add assembly="ESRI . ArcGIS.ADF . Web. DataSources,

Version=9.2.1.1332, Culture=neutral, PublickeyToken=8FC3CC631E44AD86"/> <add assembly="ESRI. ArcGIS.Geometry, Version=9.2.0.1324,

Culture=neutral, PublickeyToken=8FC3CC631E44AD86"/>

$<$ add assembly="ESRI. ArcGIS.Server, Version=9.2.0.1324,

Culture=neutral, PublickeyToken=8FC3CC631E44AD86"/> <add assembly="ESRI. ArcGIS.Geodatabase, Version=9.2.0.1324,

Culture=neutral, PublickeyToken=8FC3CC631E44AD86"/> 
$<$ add assembly="ESRI. ArcGIS.Carto, Version=9.2.0.1324,

Culture=neutral, PublickeyToken=8FC3CC631E44AD86"/>

<add assembly="ESRI.ArcGIS.ADF.Connection, Version=9.2.1.1332,

Culture=neutral, PublickeyToken=8FC3CC631E44AD86"/>

<add assembly="ESRI.ArcGIS.System, Version=9.2.0.1324,

Culture=neutral, PublickeyToken=8FC3CC631E44AD86"/> <add assembly="ESRI . ArcGIS. ADF. Web. DataSources.Graphics,

Version=9.2.1.1332, Culture=neutral, PublicKeyToken=8FC3CC631E44AD86"/> <add assembly="ESRI.ArcGIS.ADF.Tasks, Version=9.2.1.1332,

Culture=neutral, PublickeyToken=8FC3CC631E44AD86"/> <add assembly="System.Design, Version=2.๑.๑.๑, Culture=neutral,

PublickeyToken=B03F5F7F11D50A3A"/> <add assembly="System.Windows.Forms, Version=2.๑.๑.๑,

Culture=neutral, PublickeyToken=B77A5C561934E089"/> $<$ add assembly="ESRI . ArcGIS. ADF . ArcGISServer,

Version=9.2.1.1332, Culture=neutral, PublicKeyToken=8FC3CC631E44AD86"/> $<$ add assembly="ESRI . ArcGIS. ADF. Web . DataSources. ArcGISServer,

Version=9.2.1.1332, Culture=neutral, PublickeyToken=8FC3CC631E44AD86"/> $<$ add assembly="ESRI . ArcGIS . ADF . ArcGISServer . Editor,

Version=9.2.1.1332, Culture=neutral, PublickeyToken=8FC3CC631E44AD86" /> <add assembly="ESRI. ArcGIS. Location, Version=9.2.0.1324,

Culture=neutral, PublickeyToken=8FC3CC631E44AD86" $/>$

$</$ assemblies $>$

$<$ ! - -

$</$ compilation $>$

Note \#2: Codes starting from <httpHandlers> and ending

$</$ httpModules $>$ originated in the AJAX Enable Website web.config, and transferred to the Web Mapping Application web.config.

$-->$

$<$ httpHandlers $>$

$<$ remove verb="*" path="*.asmx"/>

$<$ add verb="*" path="*.asmx" validate="false"

type="System.Web.Script.Services.ScriptHandlerFactory,

System.Web.Extensions, Version=1.0.61025.๑, Culture=neutral,

PublickeyToken=31bf3856ad364e35"/>

$<$ add verb="*" path="*_AppService.axd" validate="false"

type="System. Web.Script.Services.ScriptHandlerFactory,

System.Web.Extensions, Version=1.0.61025.0, Culture=neutral,

PublicKeyToken=31bf3856ad364e35"/>

<add verb="GET, HEAD" path="ScriptResource.axd"

type="System.Web.Handlers.ScriptResourceHandler, System.Web.Extensions, Version=1.0.61025.๑, Culture=neutral, PublickeyToken=31bf3856ad364e35"

validate="false"/>

$</$ httpHandlers $>$

$<$ httpModules $>$

<add name="ScriptModule" type="System.Web.Handlers.ScriptModule, System.Web.Extensions, Version=1.0.61025.0, Culture=neutral,

PublickeyToken=31bf3856ad364e35"/>

$</$ httpModules $>$

$<!--$

End of Note \#2

$-->$

$<$ pages theme="Green_Trees" $>$

$<$ namespaces $>$

$<$ clear/>

<add namespace="System"/> 


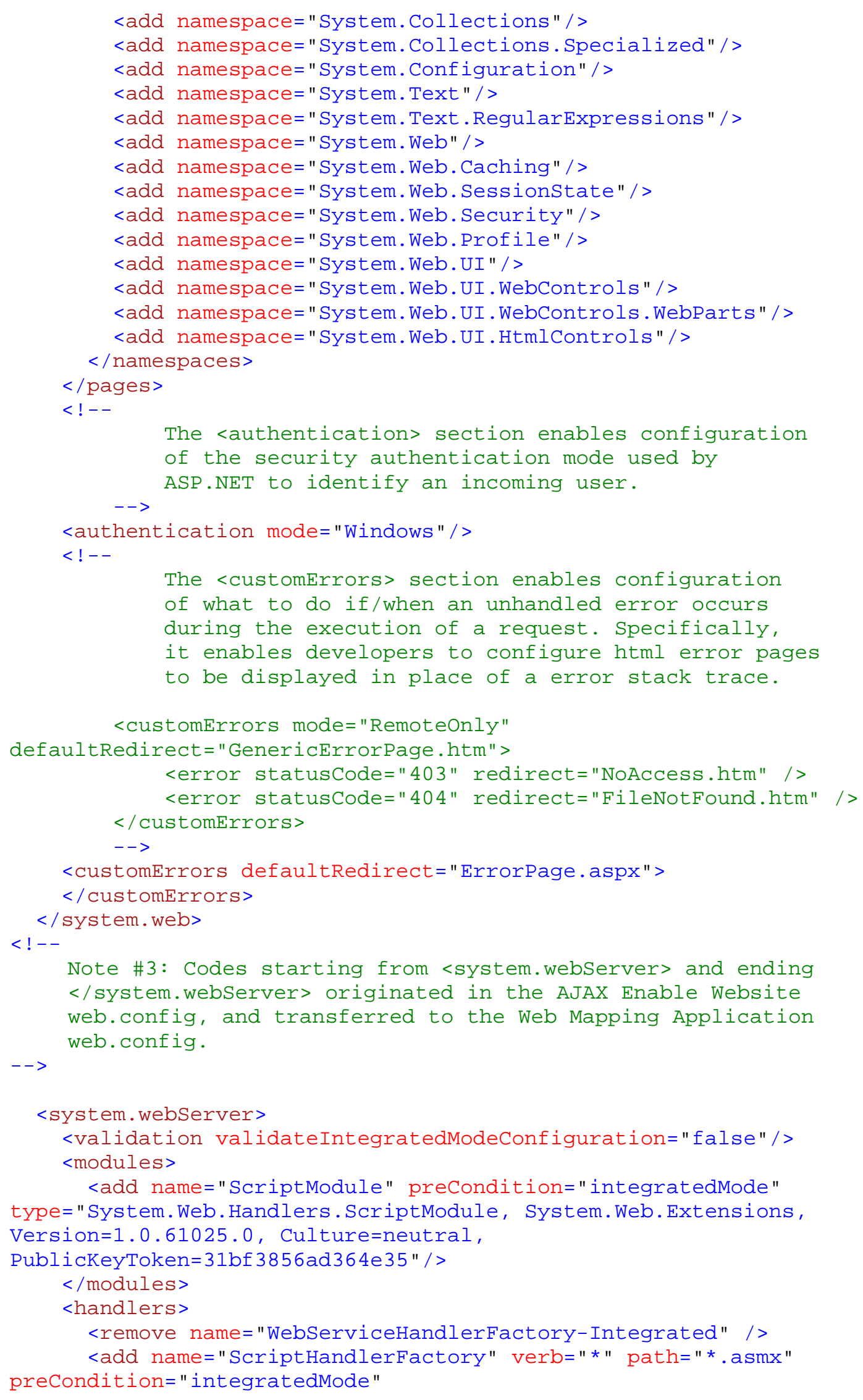


type="System. Web.Script.Services.ScriptHandlerFactory, System.Web.Extensions, Version=1.๑.61025.๑, Culture=neutral, PublickeyToken=31bf3856ad364e35"/> <add name="ScriptHandlerFactoryAppServices" verb="*" path=" *AppService.axd" preCondition="integratedMode" type="System. Web.Script.Services.ScriptHandlerFactory, System.Web.Extensions, Version=1.0.61025.0, Culture=neutral, PublickeyToken=31bf3856ad364e35"/> <add name="ScriptResource" precondition="integratedMode" verb="GET, HEAD" path="ScriptResource.axd" type="System.Web.Handlers.ScriptResourceHandler, System.Web.Extensions, Version=1.0.61025.๑, Culture=neutral, PublickeyToken=31bf3856ad364e35"

/>

$</$ handlers $>$

$</$ system. webServer $>$

$<!--$

End of Note \#3

$-->$

$</$ configuration $>$ 



\section{Appendix F - Custom Table of Contents Code}

The code in this appendix turns on and off the individual layers when checked boxes are turned on and off, respectively. The full appendix appears on the enclosed project CD.

Protected Sub chkAccommodation_CheckedChanged(ByVal sender As object, ByVal e As System.EventArgs) Handles chkAgency.CheckedChanged, chkCommunication. CheckedChanged, chkEducation.CheckedChanged, chkFinancial. CheckedChanged, chkFood.CheckedChanged, chkInformationCenter.CheckedChanged, chkMedical.CheckedChanged, chkMuseum. CheckedChanged, chkshopping. CheckedChanged, chkTourOperator. CheckedChanged, chkTransportation. CheckedChanged

Dim sLayerIDs() As String

Dim sLayerNames() As String

Dim nLayer As Integer $=-1$

'Dim mapFunction As

ESRI . ArcGIS . ADF . Web. DataSources. IGISFunctionality

Dim pMap As IMapFunctionality

If Typeof Map1.GetFunctionality( $\odot)$ Is

ESRI . ArcGIS. ADF. Web. DataSources. ArcGISServer. MapFunctionality Then

' mapFunction = CType $($ Map1.GetFunctionality $(\odot)$,

ESRI . ArcGIS. ADF . Web. DataSources. ArcGISServer . MapFunctionality)

pMap $=$ Map1. GetFunctionality $(\odot)$

Else

' mapFunction = CType(Map1.GetFunctionality(1),

ESRI . ArcGIS . ADF . Web . DataSources . ArcGISServer . MapFunctionality)

pMap $=$ Map1.GetFunctionality (1)

End If

pMap.GetLayers(sLayerIDs, sLayerNames)

For nLayer $=\odot$ To sLayerNames. GetUpperBound $(\odot)$

If sLayerNames(nLayer) = sender.text.ToString.Substring (1,

sender.text.ToString.Indexof(".")).Trim Then Exit For

Next

Dim senderControl As New System.Web.UI.WebControls.CheckBox

senderControl = CType (sender,

System. Web. UI. WebControls. CheckBox)

If senderControl.Checked Then

Else pMap.SetLayerVisibility(sLayerIDs(nLayer), True)

End If

pMap.SetLayerVisibility(sLayerIDs(nLayer), False)

Map1.Refresh()

If ScriptManager1. IsInAsyncPostBack Then

Dim callbackstring As String =

Map1.CallbackResults.ToString

ScriptManager1.RegisterDataItem(Map1, callbackString)

End If

End Sub 



\section{Appendix G - Code for the Category Drop-Down List}

The code in this appendix populates the category drop-down list with accommodation categories. The full appendix appears on the enclosed project CD.

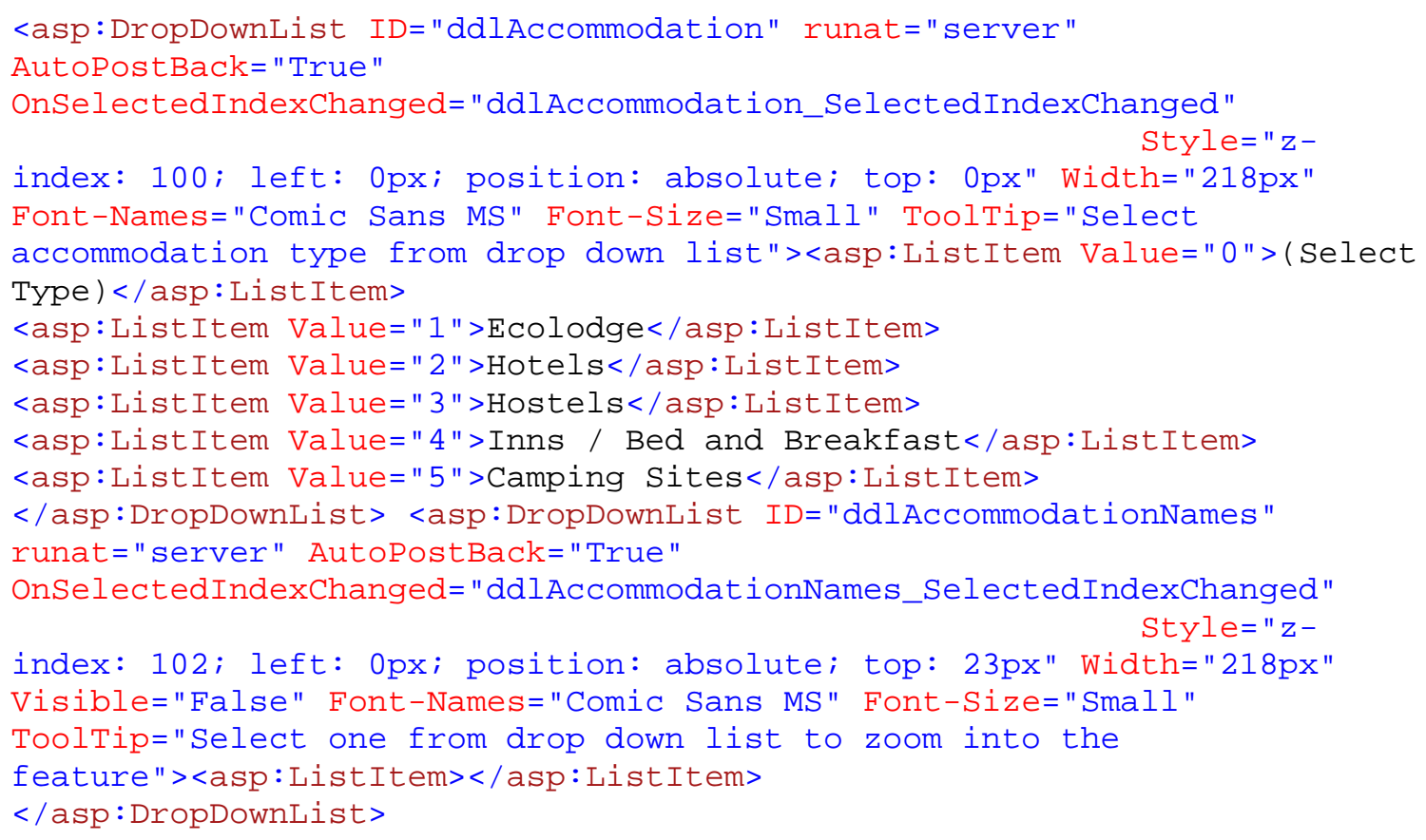





\section{Appendix H - Code for the Subcategory Drop-Down List}

The code in this appendix performs a query on the database. The full appendix appears on the enclosed project CD.

Protected Sub ddlAccommodation_SelectedIndexChanged(ByVal sender As Object, ByVal e As System.EventArgs)

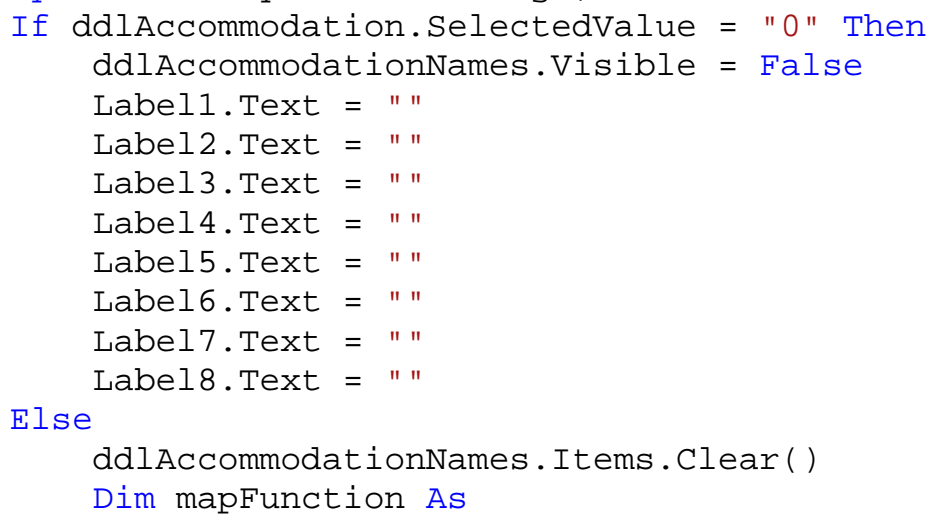

ESRI . ArcGIS . ADF . Web . DataSources . IGISFunctionality

If Typeof Map1.GetFunctionality( $\odot)$ Is

ESRI . ArcGIS. ADF. Web. DataSources. ArcGISServer. MapFunctionality Then mapFunction = CType $($ Map1. GetFunctionality $(\odot)$,

ESRI . ArcGIS. ADF . Web . DataSources. ArcGISServer . MapFunctionality) Else

mapFunction = CType(Map1.GetFunctionality(1),

ESRI . ArcGIS . ADF . Web . DataSources . ArcGISServer . MapFunctionality)

End If

Dim mapResource As

ESRI. ArcGIS.ADF. Web. DataSources.IGISResource = mapFunction. Resource Dim queryFunction As

ESRI . ArCGIS. ADF . Web. DataSources. IQueryFunctionality queryFunction $=$

CType (mapResource.CreateFunctionality (GetType(ESRI . ArcGIS . ADF . Web . DataS ources.IQueryFunctionality), Nothing),

ESRI . ArcGIS. ADF. Web. DataSources. IQueryFunctionality)

Dim queryDataTables As System. Data.DataTable

Dim queryFilter As New ESRI.ArcGIS.ADF.Web.QueryFilter

queryFilter. WhereClause $=$ "RF_CATEGORY_CD $=1$ AND

RF_SUBCATEGORY_CD = " \& ddlAccommodation.SelectedValue queryFilter) queryDataTables = queryFunction.Query(Nothing, "1",

Dim selectItem As New Listitem

selectItem. Text $=$ "( select from below $) "$

selectItem. Value $="-1 "$

ddlAccommodationNames. Items. Add(select Item)

For rowIndex As Int32 $=\odot$ To queryDataTables. Rows. Count -1

Dim queryRow As Data. DataRow

Dim newListItem As New ListItem

queryRow $=$ queryDataTables. Rows. Item (rowIndex $)$ 
newListItem. Text $=$ queryRow.Item(3). ToString

newListItem.Value $=$ queryRow. Item $(0)$. Tostring

ddlAccommodationNames. Items . Add (newList Item) Next

' (Select all locations): Added June 9

Dim selectAll As New ListItem

selectAll. Text = "(Select All Locations)"

selectAll. Value $="-2 "$

ddlAccommodationNames. Items. Add (selectAll)

' (Select all under \$10): Added June 11

Dim selectAllunderten As New ListItem

selectAllunderten. Text $=$ "(Select All Under \$10)"

selectAllunderten.Value = "-3"

ddlAccommodationNames. Items. Add(selectAllunderten)

' (Select all between \$10-\$25): Added June 26

Dim selectAll1025 As New ListItem

selectAll1025. Text $=$ "(Select All Between \$10-\$25)"

selectAll1025. Value = "-4"

ddlAccommodationNames. Items . Add (selectAll1025)

'(Select all between \$25-\$50): Added June 26

Dim selectAll5 As New ListItem

selectAll5. Text = "(Select All Between \$25-\$50)"

selectAll5. Value $="-5 "$

ddlAccommodationNames. Items . Add (selectAll5)

'(Select all between \$50-\$100): Added June 26

Dim selectAll6 As New ListItem

selectAll6. Text $=$ "(Select All Between $\$ 50-\$ 100) "$

selectAll6. Value $="-6 "$

ddlAccommodationNames. Items. Add (selectAll6)

ddlAccommodationNames.Visible = True

' (Select all over \$100): Added June 26

Dim selectAll7 As New ListItem

selectAll7. Text $=$ "(Select All Over \$100)"

selectAll7. Value $="-7 "$

ddlAccommodationNames. Items. Add (selectAll7)

End If

ddlAccommodationNames.Visible $=$ True

End Sub 


\section{Appendix I - Another Code for the Subcategory Drop-Down List}

The code in this appendix allows the map to zoom into the selected feature that was specified in the accommodation category drop-down list. The full appendix appears on the enclosed project CD.

Protected Sub ddlAccommodationNames_SelectedIndexChanged(ByVal sender As Object, Byval e As System.EventArgs)

Dim gresource As New

ESRI . ArcGIS. ADF . Web . DataSources . Graphics . MapResource

Dim fglayer As New

ESRI . ArcGIS . ADF . Web . Display . Graphics . FeatureGraphicsLayer GetGraphicsLayer (gresource, fglayer)

If ddlAccommodationNames. SelectedValue $="-1 "$ Then 'users selected ("select from below") Label1. Text = "" Label2. Text $=" "$ Labe13. Text $=" "$ Label4. Text $=" "$ Labe15. Text $=" "$ Label6. Text $=" "$ Label7. Text $=" "$ Label8. Text $=" "$

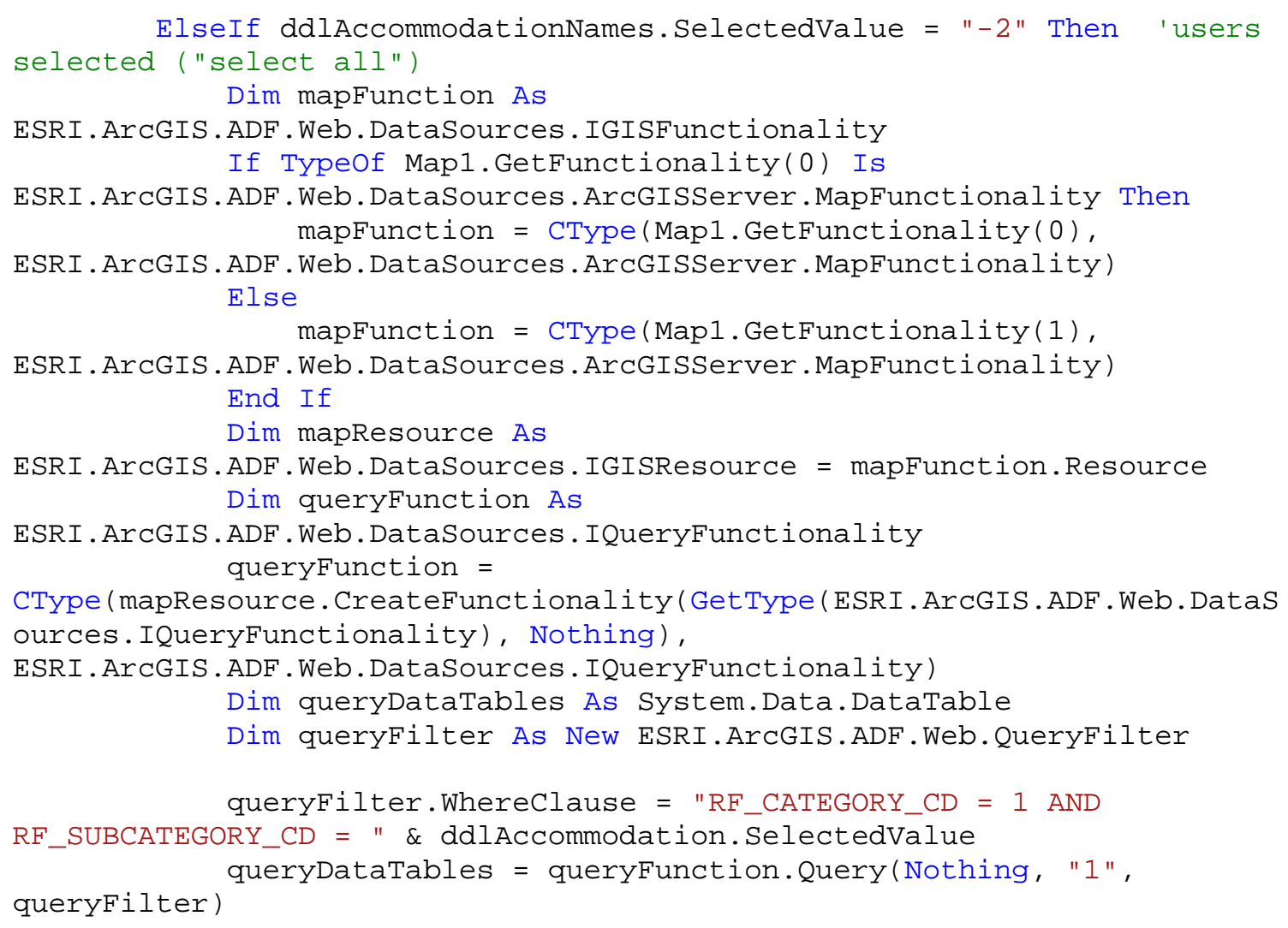


ReDim multi(queryDataTables.Rows.Count - 1)

For rowIndex As Integer $=0$ To queryDataTables. Rows. Count -

1

Dim queryRow As Data.DataRow

queryRow $=$ queryDataTables. Rows. Item (rowIndex)

multi (rowIndex) = queryRow.Item(1)

multiNames $+=$ queryRow.Item(3) \& ", "

Next

If multiNames. Length $>1$ Then multiNames $=$

multiNames.Substring $(\odot$, multiNames.LastIndexof $(", "))$

Map1.ZoomToFullExtent ( )

For $i$ As Int32 $=\odot$ To multi.GetUpperBound $(\odot)$

Next

fglayer.Add(multi(i))

Labe19. Text $=" "$

Label10. Text $=" "$

Label1.Text $=$ (multi.GetUpperBound $(\odot)+1)$.ToString \& "

location(s) selected"

Label2. Text = multiNames

Label3. Text $=" "$

Label4. Text $=" "$

Labe15. Text $=" "$

Label6. Text $=" "$

Labe17. Text $=" "$

Label8. Text $=" "$

Map1.Refresh( )

ElseIf ddlAccommodationNames.SelectedValue $=$ "-3" Then 'users selected ("select all under \$10")

Dim mapFunction As

ESRI . ArcGIS . ADF . Web. DataSources . IGISFunctionality

If Typeof Map1.GetFunctionality $(\odot)$ Is

ESRI . ArcGIS. ADF. Web. DataSources. ArcGISServer. MapFunctionality Then mapFunction = CType $($ Map1.GetFunctionality $(\odot)$,

ESRI . ArcGIS . ADF . Web . DataSources . ArcGISServer . MapFunctionality) Else

mapFunction = CType(Map1.GetFunctionality(1),

ESRI . ArcGIS . ADF. Web . DataSources . ArcGISServer . MapFunctionality)

End If

Dim mapResource As

ESRI . ArcGIS. ADF. Web. DataSources. IGISResource = mapFunction. Resource Dim queryFunction As

ESRI . ArcGIS . ADF . Web . DataSources . IQueryFunctionality queryfunction =

CType (mapResource.CreateFunctionality (GetType(ESRI . ArcGIS. ADF . Web . DataS ources.IQueryFunctionality), Nothing),

ESRI . ArCGIS. ADF. Web. DataSources. IQueryFunctionality)

Dim queryDataTables As System.Data.DataTable

Dim queryFilter As New ESRI.ArcGIS.ADF.Web.QueryFilter

queryfilter. Whereclause $=$ "PRICE_CD $=1$ AND RF_CATEGORY_CD

$=1$ AND RF_SUBCATEGORY_CD $="$ " \& ddlAccommodation. SelectedValue

queryfilter) queryDataTables = queryFunction.Query(Nothing, "1", 
Dim multi() As ESRI.ArcGIS.ADF.Web.Geometry.Point

Dim multiNames As String = ""

ReDim multi(queryDataTables. Rows. Count - 1)

1

For rowIndex As Integer $=0$ To queryDataTables. Rows. Count -

Dim queryRow As Data.DataRow

queryRow $=$ queryDataTables. Rows. Item (rowIndex $)$

multi (rowIndex) = queryRow. Item (1)

Next

multiNames $+=$ queryRow.Item(3) \& ", "

If multiNames. Length $>1$ Then multiNames $=$

multiNames.Substring $(\odot$, multiNames.LastIndexof $(", "))$

Map1.ZoomToFullExtent ( )

For $i$ As Int32 $=\odot$ To multi.GetUpperBound $(\odot)$

Next fglayer.Add(multi(i))

Labe19. Text $=" "$

Label10. Text $=" "$

location(s) selected"

Label1.Text $=($ multi.GetUpperBound $(0)+1)$.ToString \& "

Label2. Text $=$ multiNames

Labe13. Text $=" "$

Label4.Text $=" "$

Labe15. Text $=" "$

Label6. Text $=" "$

Labe17. Text $=" "$

Label8. Text $=" "$

Map1.Refresh( )

ElseIf ddlAccommodationNames.SelectedValue $=$ "-4" Then 'users

selected ("select all between $\$ 10-\$ 25 "$ )

Dim mapFunction As

ESRI . ArcGIS . ADF . Web. DataSources. IGISFunctionality

If Typeof Map1.GetFunctionality $(\odot)$ Is

ESRI . ArcGIS. ADF. Web. DataSources. ArcGISServer. MapFunctionality Then mapFunction = CType $($ Map1. GetFunctionality $(\odot)$,

ESRI . ArCGIS . ADF . Web . DataSources . ArcGISServer . MapFunctionality) Else

mapFunction = CType(Map1.GetFunctionality(1),

ESRI . ArcGIS . ADF . Web . DataSources . ArcGISServer . MapFunctionality)

End If

Dim mapResource As

ESRI . ArcGIS.ADF. Web. DataSources. IGISResource = mapFunction. Resource Dim queryFunction As

ESRI . ArCGIS . ADF . Web. DataSources. IQueryFunctionality queryFunction $=$

CType (mapResource.CreateFunctionality (GetType(ESRI . ArcGIS . ADF . Web . DataS ources.IQueryFunctionality), Nothing),

ESRI . ArCGIS. ADF. Web . DataSources. IQueryFunctionality)

Dim queryDataTables As System. Data.DataTable

Dim queryFilter As New ESRI.ArcGIS.ADF.Web.QueryFilter

queryfilter.whereclause $=$ "PRICE_CD $=2$ AND RF_CATEGORY_CD

$=1$ AND RF_SUBCATEGORY_CD = " \& ddlAccommodation.SelectedValue 
queryfilter)

queryDataTables = queryFunction.Query(Nothing, "1",

Dim multi() As ESRI.ArcGIS.ADF.Web.Geometry.Point

Dim multiNames As String = ""

ReDim multi(queryDataTables. Rows. Count - 1)

1

For rowIndex As Integer $=0$ To queryDataTables. Rows. Count -

Dim queryRow As Data.DataRow

queryRow = queryDataTables. Rows. Item (rowIndex)

multi (rowIndex) = queryRow.Item(1)

multiNames $+=$ queryRow.Item(3) \& ", "

Next

If multiNames. Length $>1$ Then multiNames $=$

multiNames.Substring $(\odot$, multiNames.LastIndexof $(", "))$

Map1.ZoomToFullExtent ( )

For $i$ As Int32 $=\odot$ To multi.GetUpperBound $(\odot)$

Next

fglayer.Add(multi(i))

Labe19. Text $=" "$

Label10. Text $=" "$

Label1. Text $=($ multi.GetUpperBound $(\odot)+1)$. ToString \& "

location(s) selected"

Label2. Text $=$ multiNames

Labe13. Text $=" "$

Label4. Text $=" "$

Labe15. Text $=" "$

Label6. Text $=" "$

Label7. Text = ""

Label8. Text $=" "$

Map1.Refresh( )

ElseIf ddlAccommodationNames.SelectedValue $=$ "-5" Then 'users

selected ("select all between \$25-\$50")

Dim mapFunction As

ESRI . ArcGIS. ADF . Web. DataSources. IGISFunctionality

If Typeof Map1.GetFunctionality( $\odot)$ Is

ESRI . ArcGIS. ADF. Web. DataSources. ArcGISServer. MapFunctionality Then mapFunction = CType $($ Map1.GetFunctionality $(0)$,

ESRI . ArcGIS . ADF . Web . DataSources . ArcGISServer . MapFunctionality) Else

mapFunction = CType(Map1.GetFunctionality(1),

ESRI . ArcGIS . ADF . Web . DataSources . ArcGISServer . MapFunctionality)

End If

Dim mapResource As

ESRI. ArcGIS. ADF. Web. DataSources. IGISResource = mapFunction. Resource Dim queryFunction As

ESRI . ArCGIS . ADF . Web . DataSources. IQueryFunctionality queryfunction =

CType (mapResource.CreateFunctionality (GetType(ESRI . ArcGIS . ADF . Web . DataS ources.IQueryFunctionality), Nothing),

ESRI . ArcGIS. ADF. Web . DataSources. IQueryFunctionality)

Dim queryDataTables As System. Data.DataTable

Dim queryFilter As New ESRI.ArcGIS.ADF.Web.QueryFilter 
queryfilter.WhereClause $=$ "PRICE_CD $=3$ AND RF_CATEGORY_CD $=1$ AND RF_SUBCATEGORY_CD $=" \&$ ddlAccommodation.SelectedValue queryFilter) queryDataTables = queryFunction.Query(Nothing, "1",

Dim multi() As ESRI.ArcGIS.ADF.Web.Geometry.Point Dim multiNames As String = ""

ReDim multi(queryDataTables. Rows. Count - 1)

1

For rowIndex As Integer $=0$ To queryDataTables. Rows. Count -

Dim queryRow As Data. DataRow

queryRow = queryDataTables. Rows. Item (rowIndex) multi $($ rowIndex $)=$ queryRow. Item(1)

Next

multiNames $+=$ queryRow. Item(3) \& ", "

If multiNames. Length $>1$ Then multiNames $=$

multiNames.Substring $(\odot$, multiNames.LastIndexof $(", "))$

Map1.ZoomToFullExtent ( )

For $i$ As Int32 $=\odot$ To multi.GetUpperBound $(\Theta)$

Next fglayer.Add(multi(i))

Labe19. Text $=" "$

Label10.Text $=" "$

Label1. Text $=$ (multi.GetUpperBound $(\theta)+1)$. ToString \& "

location(s) selected"

Label2. Text $=$ multiNames

Labe13.Text $=" "$

Labe14. Text $=" "$

Label5. Text $=" "$

Label6. Text $=" "$

Labe17. Text $=" "$

Label8. Text $=" "$

Map1.Refresh( )

ElseIf ddlAccommodationNames.SelectedValue $="-6 "$ Then 'users

selected ("select all between $\$ 50-\$ 100 "$ )

Dim mapFunction As

ESRI . ArcGIS . ADF . Web . DataSources. IGISFunctionality

If Typeof Map1.GetFunctionality $(\odot)$ Is

ESRI . ArcGIS. ADF. Web. DataSources. ArcGISServer. MapFunctionality Then mapFunction = CType $($ Map1. GetFunctionality $(\odot)$,

ESRI . ArcGIS. ADF . Web . DataSources . ArcGISServer . MapFunctionality) Else

mapFunction = CType (Map1.GetFunctionality(1),

ESRI . ArcGIS . ADF . Web . DataSources . ArcGISServer . MapFunctionality)

End If

Dim mapResource As

ESRI ArCGIS.ADF.Web.DataSources.IGISResource = mapFunction. Resource Dim queryFunction As

ESRI . ArCGIS . ADF . Web. DataSources. IQueryFunctionality queryfunction =

CType (mapResource.CreateFunctionality (GetType (ESRI . ArcGIS . ADF . Web . DataS ources.IQueryFunctionality), Nothing),

ESRI . ArcGIS. ADF. Web . DataSources. IQueryFunctionality)

Dim queryDataTables As System.Data.DataTable 
Dim queryFilter As New ESRI.ArcGIS.ADF.Web.QueryFilter

queryfilter. Whereclause $=$ "PRICE_CD $=4$ AND RF_CATEGORY_CD

$=1$ AND RF_SUBCATEGORY_CD $="$ \& ddlAccommodation. SelectedValue queryfilter)

queryDataTábles = queryFunction.Query (Nothing, "1",

1

Dim multi() As ESRI.ArcGIS.ADF.Web.Geometry.Point

Dim multiNames As String = ""

ReDim multi(queryDataTables. Rows. Count - 1)

For rowIndex As Integer $=0$ To queryDataTables. Rows. Count -

Dim queryRow As Data.DataRow

queryRow $=$ queryDataTables. Rows. Item $($ rowIndex $)$

multi $($ rowIndex $)=$ queryRow. Item(1)

multinames $+=$ queryRow.Item(3) \& ", "

Next

If multiNames. Length $>1$ Then multiNames $=$

multiNames.Substring $(\odot$, multiNames.LastIndexof $(", "))$

Map1.ZoomToFullExtent ( )

For $i$ As Int32 $=0$ To multi.GetUpperBound $(\odot)$

Next

fglayer.Add(multi(i))

Labe19. Text $=" "$

Label10.Text $=" "$

Label1. Text $=($ multi.GetUpperBound $(\odot)+1)$. ToString \& "

location(s) selected"

Label2. Text $=$ multiNames

Label3. Text $=" "$

Label4. Text $=" "$

Label5. Text $=" "$

Label6. Text $=" "$

Label7. Text $=" "$

Label8. Text = ""

Map1.Refresh( )

ElseIf ddlAccommodationNames.SelectedValue $="-7 "$ Then 'users

selected ("select all over \$100")

Dim mapFunction As

ESRI . ArcGIS . ADF . Web . DataSources . IGISFunctionality

If Typeof Map1.GetFunctionality $(0)$ Is

ESRI. ArcGIS. ADF. Web. DataSources. ArcGISServer. MapFunctionality Then

mapFunction = CType $($ Map1. GetFunctionality $(\odot)$,

ESRI . ArcGIS . ADF . Web . DataSources . ArcGISServer . MapFunctionality) Else

mapFunction = CType (Map1.GetFunctionality(1),

ESRI . ArcGIS . ADF . Web . DataSources . ArcGISServer . MapFunctionality)

End If

Dim mapResource As

ESRI . ArcGIS. ADF. Web. DataSources. IGISResource = mapFunction. Resource Dim queryFunction As

ESRI . ArCGIS . ADF . Web . DataSources . IQueryFunctionality queryfunction =

CType (mapResource.CreateFunctionality (GetType(ESRI . ArcGIS. ADF . Web. DataS 


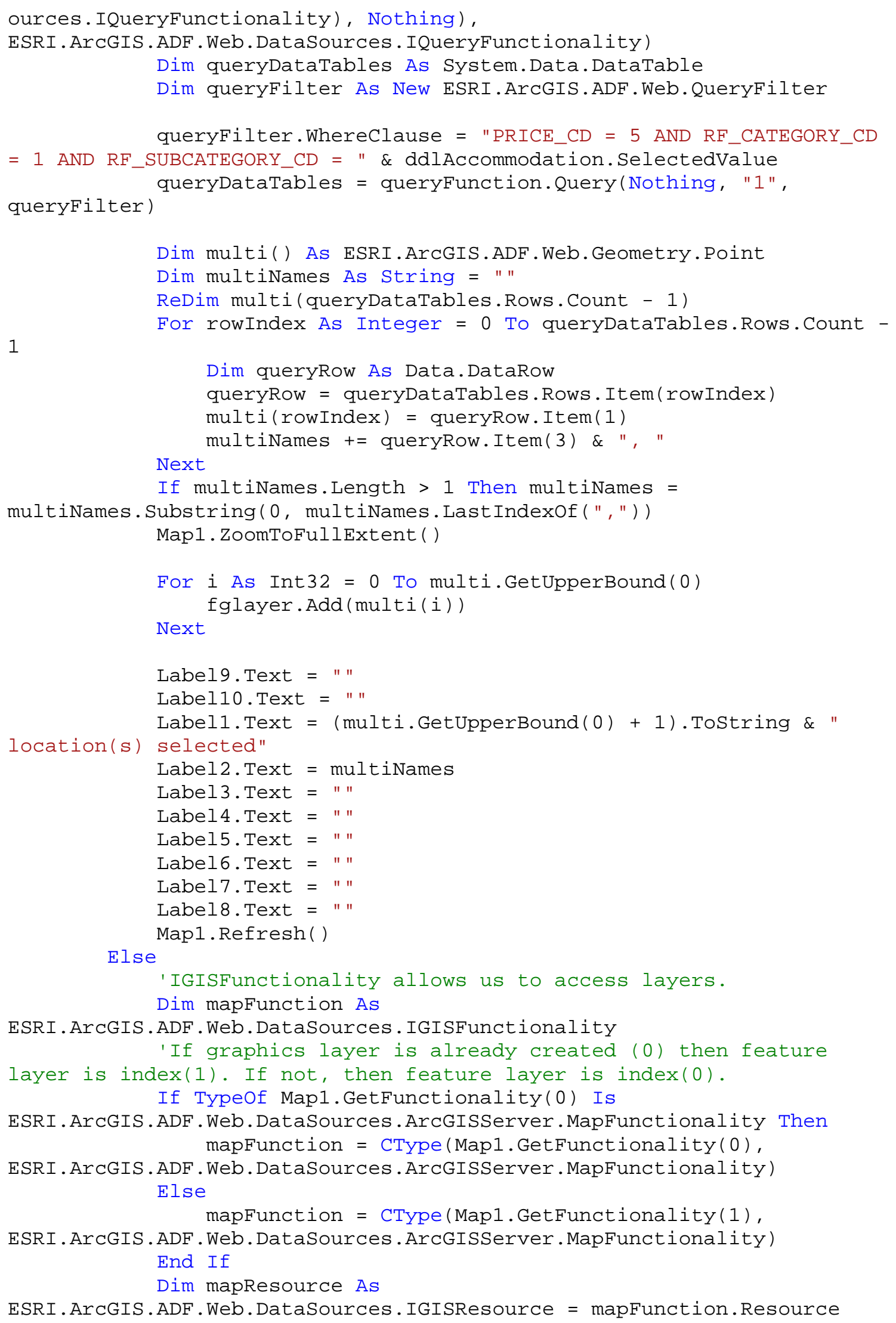


Dim queryfunction As

ESRI . ArcGIS. ADF . Web. DataSources. IQueryFunctionality

queryfunction =

CType (mapResource.CreateFunctionality (GetType (ESRI . ArcGIS. ADF . Web . DataS ources.IQueryFunctionality), Nothing),

ESRI . ArCGIS. ADF. Web. DataSources. IQueryFunctionality)

Dim queryDataTables As System. Data.DataTable

Dim queryFilter As New ESRI.ArCGIS.ADF.Web.QueryFilter

'The query filter on OBJECTID will return one feature only. queryfilter. Whereclause $=$ "OBJECTID $="$ \&

CInt (ddlAccommodationNames. SelectedValue) queryfilter)

queryDataTables = queryFunction.Query(Nothing, "1",

Dim selectedPoint As ESRI.ArcGIS.ADF.Web.Geometry.Point

Dim queryRow As Data.DataRow

queryRow $=$ queryDataTables. Rows. Item $(\odot)$

'Display additional information on the selected item

selectedPoint = CType (queryRow.Item(1),

ESRI . ArcGIS . ADF . Web . Geometry . Point)

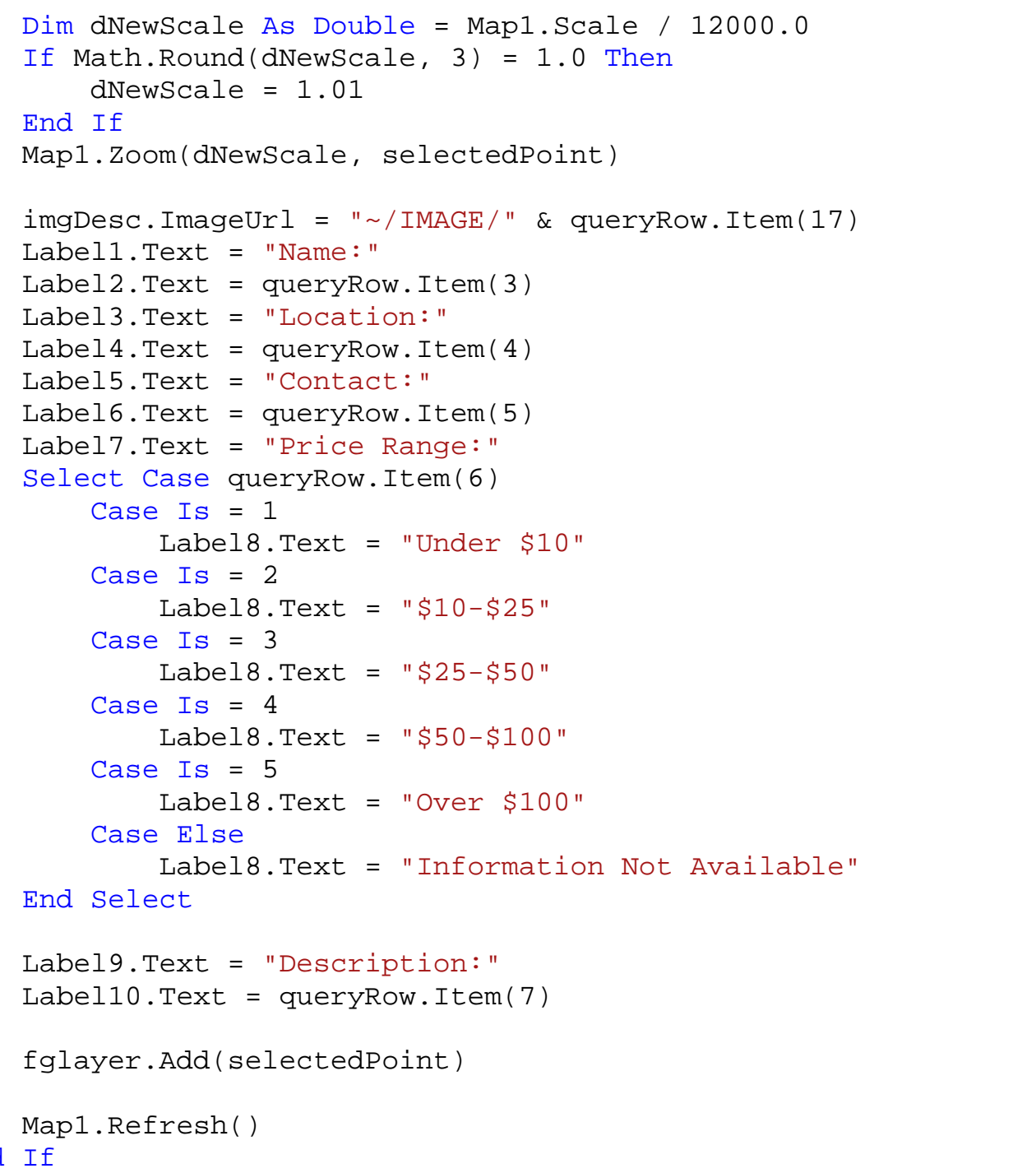


gresource.Graphics. Tables. Add (fglayer)

Map1.RefreshResource(gresource. Name)

If ScriptManager1. IsInAsyncPostBack Then Dim callbackstring As String =

Map1.CallbackResults. ToString

ScriptManager1.RegisterDataItem(Map1, callbackString) End If

End Sub 



\section{Appendix J - Installation Guide}

The instruction in this appendix is the installation guide that describes the procedures to make the two websites available to the public online. The full appendix appears on the enclosed CD: WebsiteInstallation.doc

\section{Using ArcCatalog and Administrative Tools: Internet Information Services (IIS).}

1. Copy the following three folders that appear on the project CD and paste them inside the wwwroot directory (e.g. C:IInetpub\wwwroot\Students\ChisaNishii).
(a) EcotourismServices
(b) ProtectedAreasTrails
(c) SustainableEcotourism

2. Publish the following two map documents inside the SustainableEcotourism folder by right-clicking on them individually in ArcCatalog > Publish to ArcGIS Server.

(a) EcotourismServices.mxd

(b) ProtectedAreasTrails.mxd

3. Start $>$ Control Panel $>$ Administrative Tools $>$ Internet Information Services.

4. Navigate to the two folders previously copied in step one:

(a) EcotourismServices

(b) ProtectedAreasTrails

5. Right click on the folders individually and click to see the Property. Below is the ArcGIS Properties box.

6. In the Directory tab - Application Setting, click on Create as circled in the figure below.

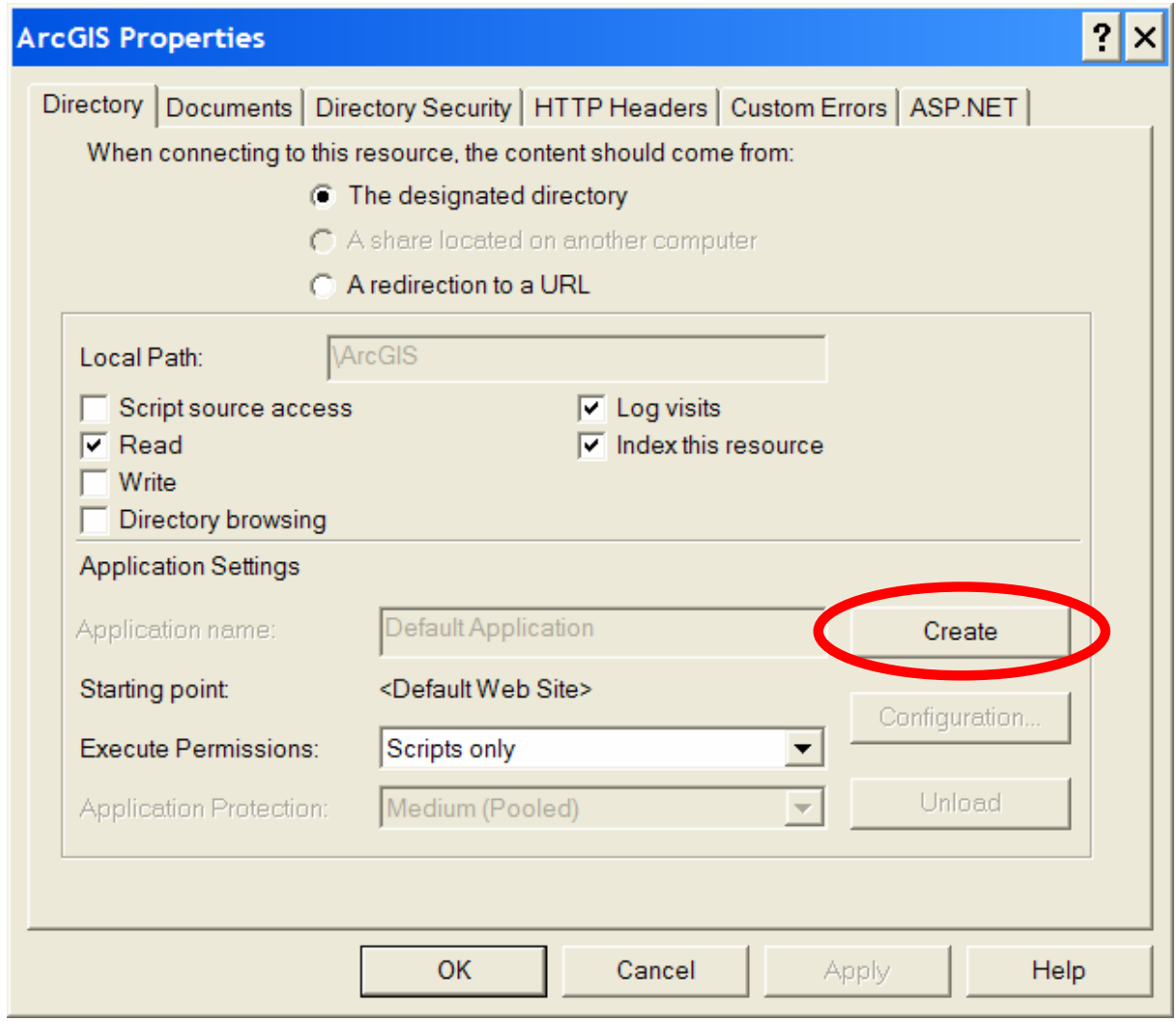




\section{Using Microsoft Visual Studio 2005: ArcGIS Identity}

\section{Website \#1: Protected Areas Trails Website}

1. Open the ProtectedAreasTrails website in Microsoft Visual Studio 2005: File $>$ Open $>$ Website $>$ ProtectedAreasTrails.

2. Open the web.config file by double clicking on it in the Solution Explorer (see $\mathbf{1}$ below).

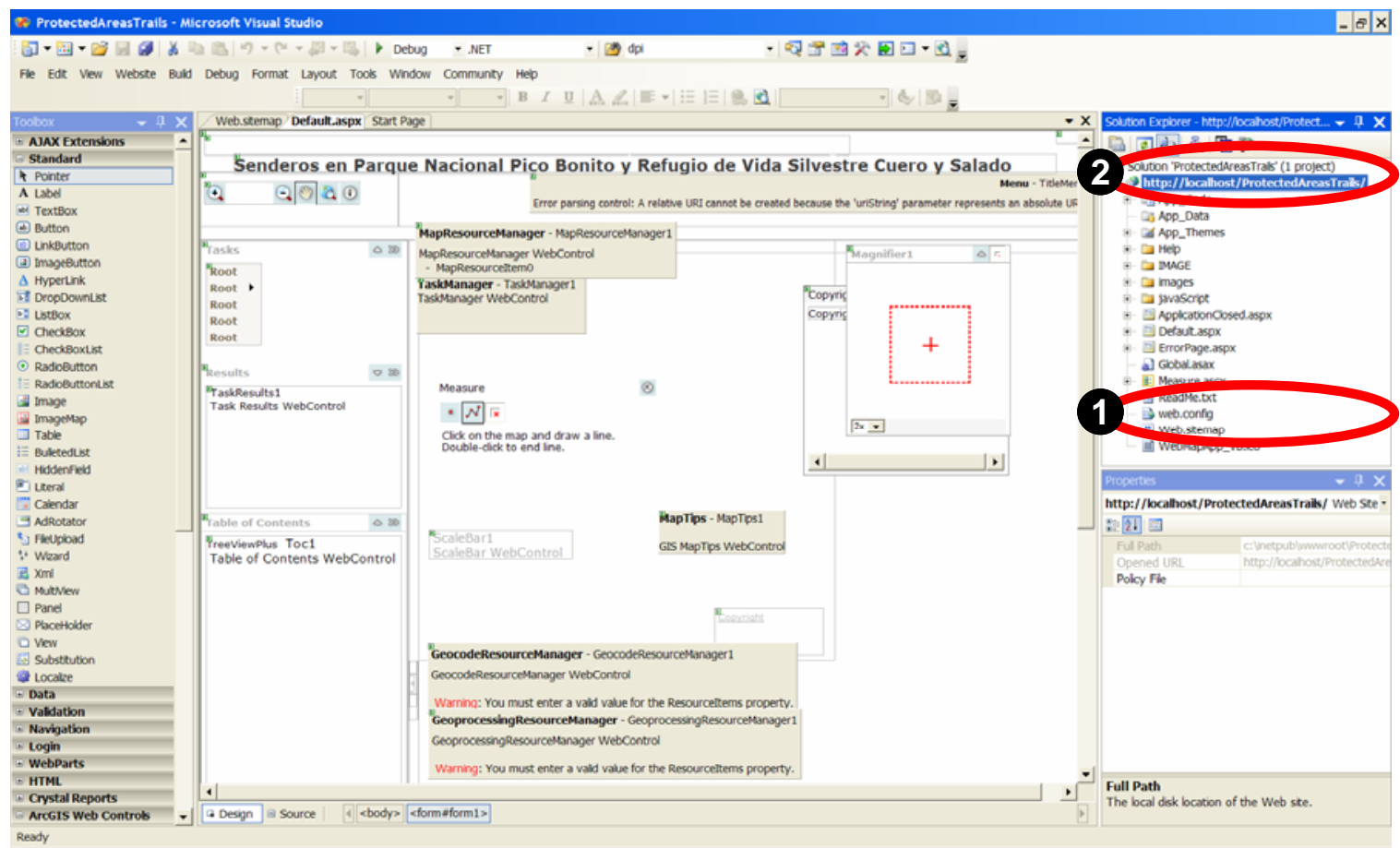

3. Delete lines of codes in between the following:

<identity ...>

$</$ identity $>$

4. In the Solution Explorer, right click on the web application (see 2 above): http://localhost/ProtectedAreasTrails/ > Add ArcGIS Identity.

\begin{tabular}{|c|c|c|}
\hline \multicolumn{3}{|c|}{ Add ArcGIS Identity } \\
\hline \multicolumn{3}{|c|}{$\begin{array}{l}\text { ArcGIS Identity } \\
\text { Type the user name, password, and domain for }\end{array}$} \\
\hline User Name: & chisa_nishii & \\
\hline Password: & 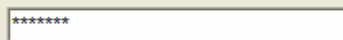 & \\
\hline \multirow[t]{3}{*}{ Domain or } & lucyl & \\
\hline & $\sqrt{V}$ Encrypt identity in web.config & \\
\hline & $\mathrm{OK}$ & Cancel \\
\hline
\end{tabular}




\section{Website \#2: Ecotourism Services Website}

1. Add ArcGIS Identity (repeat the previous processes 1-4).

2. Open the MapResourceManager.

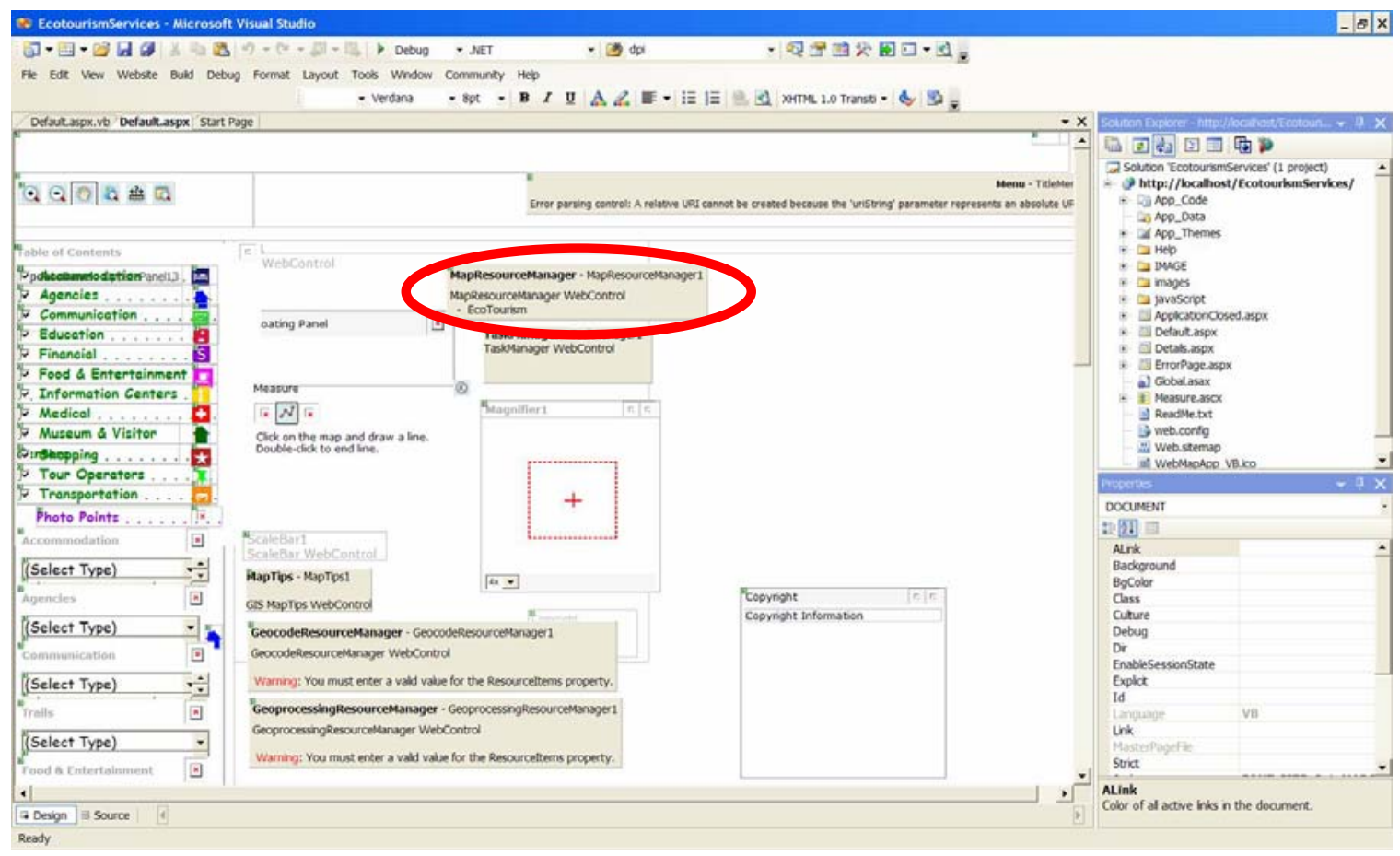

3. Click on Definition.

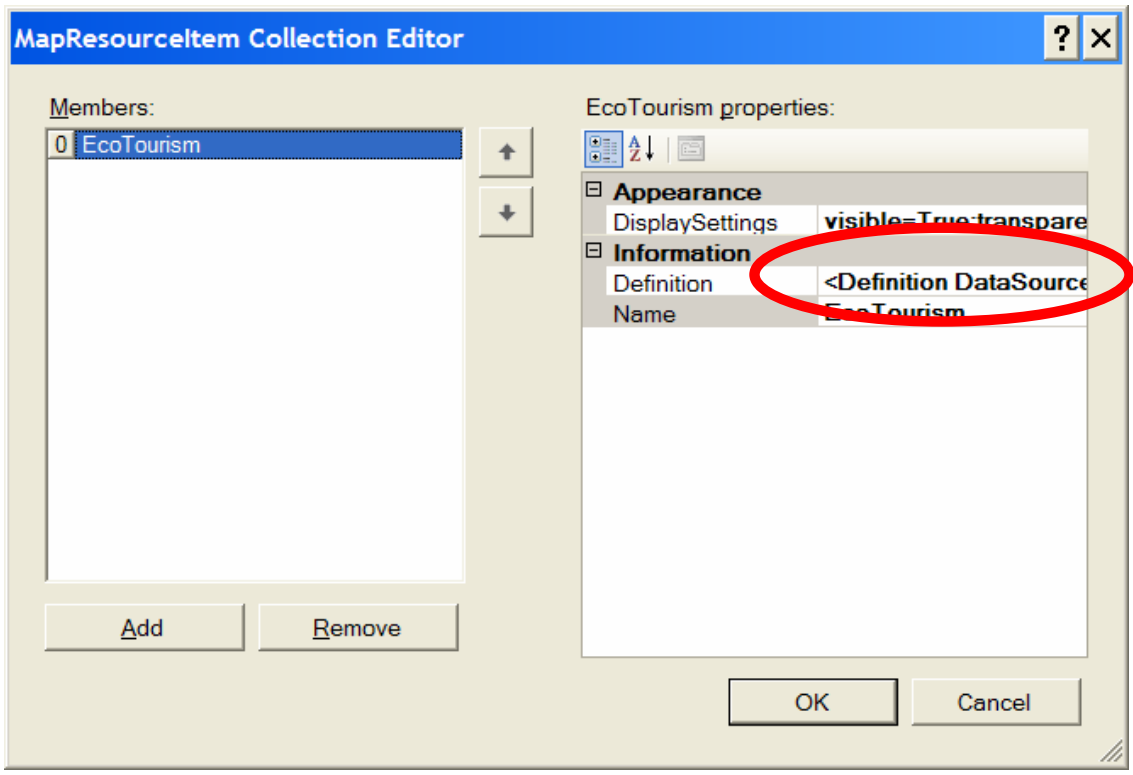


4. Make sure Data Source has the appropriate server name.

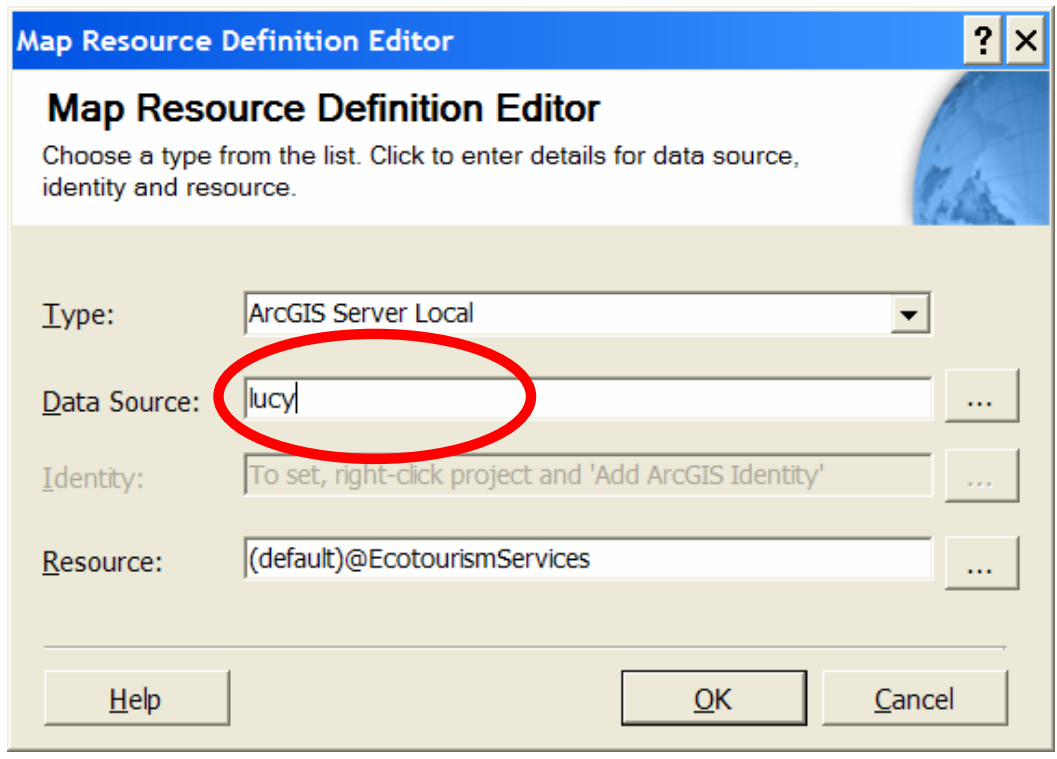

5. Open the MapTips property.

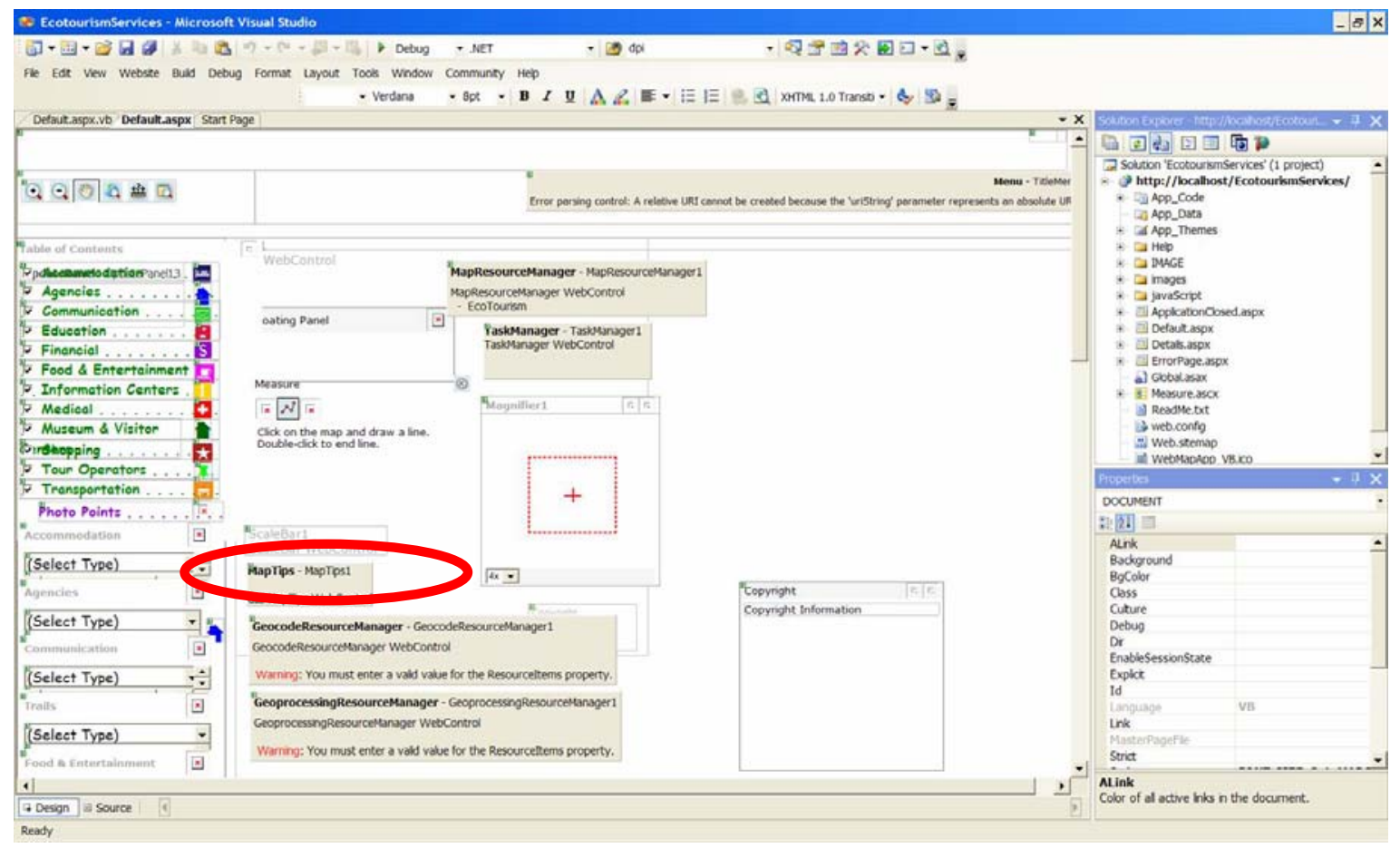


6. Single click on MapTips and single click on the black triangle on the top-right corner of the MapTips.

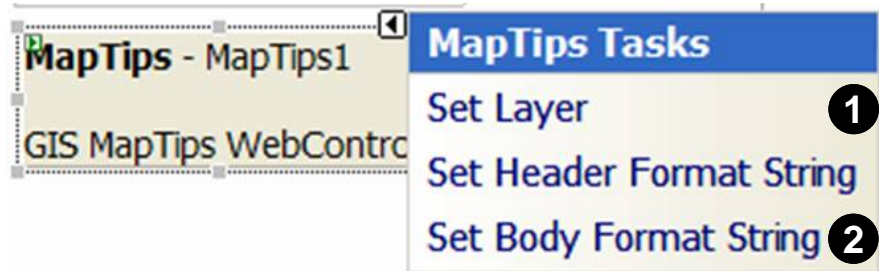

7. For Layer (MapTips > Set Layer), select PointsInterest.

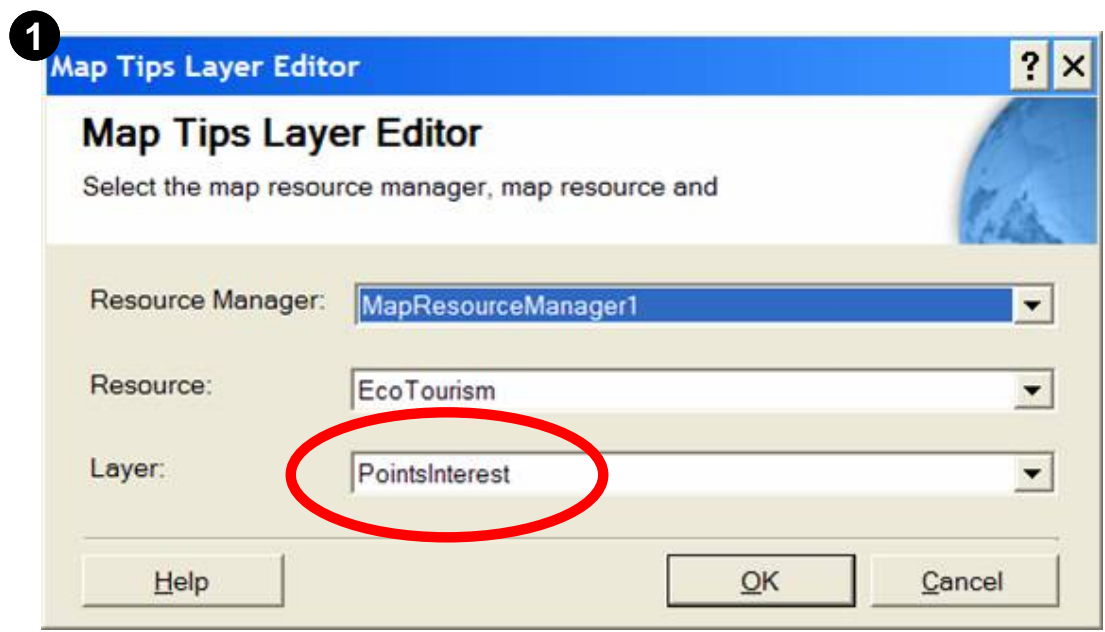

8. Make sure the path to the images are correct

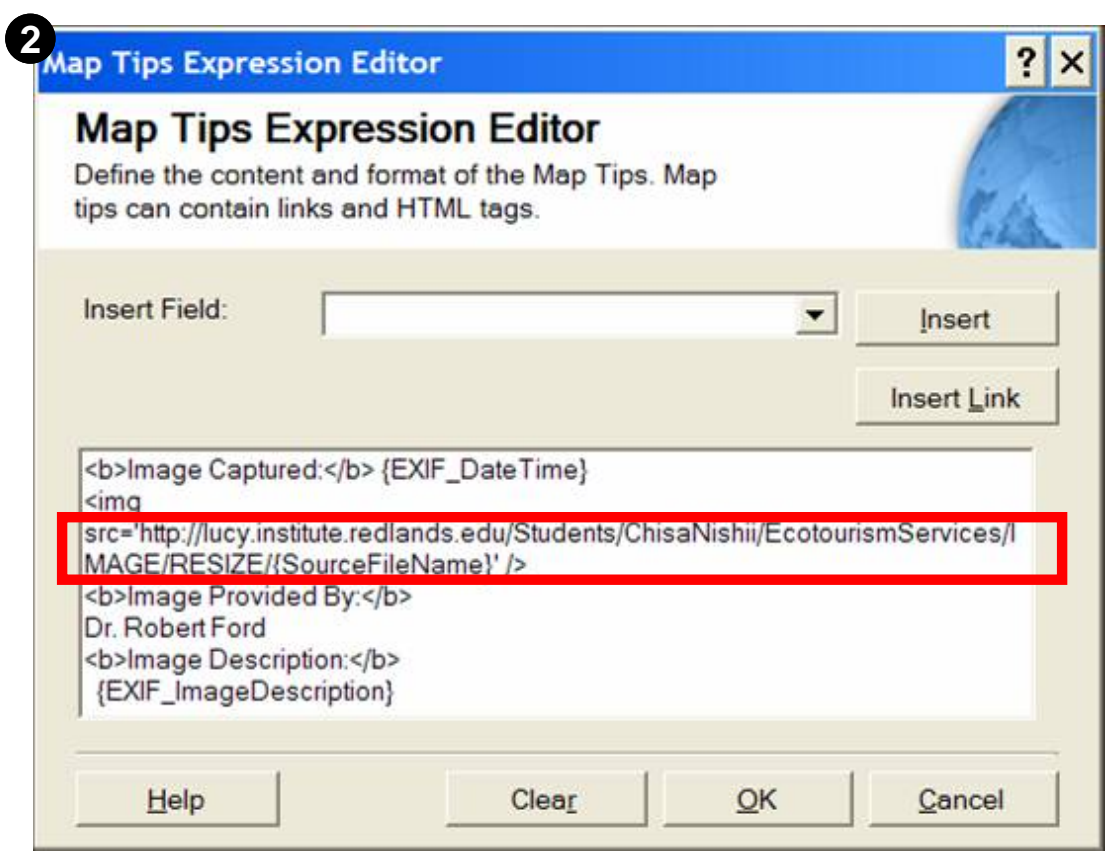

